\title{
The scaling hypothesis for Smoluchowski's coagulation equation with bounded perturbations of the constant kernel
}

\author{
José A. Cañizo* Sebastian Throm*
}

October 2019

\begin{abstract}
We consider Smoluchowski's coagulation equation with a kernel of the form $K=2+\epsilon W$, where $W$ is a bounded kernel of homogeneity zero. For small $\epsilon$, we prove that solutions approach a universal, unique self-similar profile for large times, at almost the same speed as the constant kernel case (the speed is exponential when self-similar variables are considered). All the constants we use can be explicitly estimated. Our method is a constructive perturbation analysis of the equation, based on spectral results on the linearisation of the constant kernel case. To our knowledge, this is the first time the scaling hypothesis can be fully proved for a family of kernels which are not explicitly solvable.
\end{abstract}

\section{Contents}

1 Introduction 2

2 Preliminaries $\quad 5$

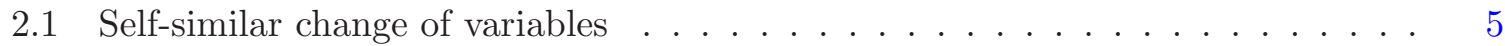

2.2 Scale invariances . . . . . . . . . . . . . . . . . 6

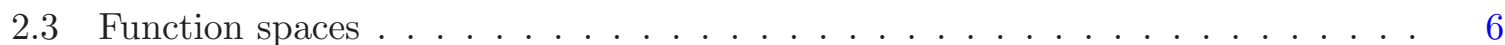

2.4 Continuity properties of $\mathcal{C}_{K} \ldots \ldots \ldots \ldots \ldots$

2.5 Existence of time-dependent solutions and moment estimates . . . . . . . . . . 9

2.6 Stability of time-dependent solutions with respect to perturbations . . . . . . . 10

2.7 Asymptotic behaviour of solutions for the constant kernel . . . . . . . . . . . 11

3 Bounds and stability of self-similar profiles $\quad 13$

3.1 Existence of self-similar profiles . . . . . . . . . . . . . . . . . . . 13

3.2 Bounds on self-similar profiles . . . . . . . . . . . . . . . . . . . . 14

3.3 Behaviour of profiles close to zero and a uniform $L^{2}$ estimate . . . . . . . . 15

3.4 Stability of profiles . . . . . . . . . . . . . . . . . . 19

4 The linearised operator for the constant kernel and semigroup theory 21

* Departamento de Matemática Aplicada, Universidad de Granada, 18071 Granada, Spain. Email addresses: canizo@ugr.es, throm@correo.ugr.es 
5 Tools on the spectral gap of linear operators 23

5.1 Spectral gap . . . . . . . . . . . . . . . . . . . . 24

5.2 Restriction of the spectral gap . . . . . . . . . . . . . . . . . 24

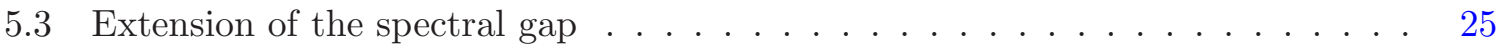

6 Spectral gap for the constant kernel in weighted $L^{1}$ spaces $\quad 26$

6.1 Restriction of the spectral gap to weigthed $L^{2}$ spaces . . . . . . . . . . . 26

6.2 Extension of the spectral gap to weighted $L^{1}$ spaces $\ldots \ldots \ldots \ldots$

7 Uniqueness of profiles $\quad 33$

7.1 Local stability of profiles . . . . . . . . . . . . . . . . . 33

7.2 Uniqueness . . . . . . . . . . . . . . . . . . . . . 35

$\begin{array}{lll}8 & \text { Convergence to equilibrium } & 37\end{array}$

8.1 Local convergence to equilibrium . . . . . . . . . . . . . . . . . 37

8.2 Convergence to equilibrium in large regions $\ldots \ldots \ldots \ldots$

A Proof of Theorem 2.16 on $L^{2}$ convergence for the constant kernel 40

B An estimate on the Fourier transform 41

C Proof of Lemma 4.6 on the transport semigroup 44

\section{Introduction}

We study the long-time behaviour of solutions to Smoluchowski's coagulation equation, which reads

$$
\partial_{\tau} \phi(\tau, \xi)=\frac{1}{2} \int_{0}^{\xi} K(\xi-\eta, \eta) \phi(\tau, \xi-\eta) \phi(\tau, \eta) \mathrm{d} \eta-\phi(\tau, \xi) \int_{0}^{\infty} K(\xi, \eta) \phi(\tau, \eta) \mathrm{d} \eta .
$$

This equation is a well-known model for coagulation processes in several contexts such as aerosol dynamics [14,31], aggregation in planetary formation [3,32] and biology [1,2]. The unknown $\phi=\phi(\tau, \xi) \geq 0$ represents the density of clusters of size $\xi>0$ at time $\tau \geq 0$, and $K=K(\xi, \eta)=K(\eta, \xi) \geq 0$ is the symmetric coagulation kernel giving the coagulation rate of clusters of size $\xi$ with clusters of size $\eta$. We always consider the continuum version of this equation, so the size $\xi$ can take any positive value. A long-standing conjecture is that all (finite-mass, suitably decaying) solutions to (1.1) approach a universal self-similar shape as time $\tau \rightarrow+\infty$, as long as $K(\xi, \eta)$ is a homogeneous function of homogeneity degree $\gamma \leq 1$ (i.e., $K(\lambda \xi, \lambda \eta)=\lambda^{\gamma} K(\xi, \eta)$ for all $\left.\lambda, \xi, \eta \in(0, \infty)\right)$; this is known as the scaling hypothesis. More precisely, one expects that there exists a self-similar profile $G$ and a scaling function $s(\tau) \rightarrow \infty$ as $\tau \rightarrow \infty$ such that

$$
(s(\tau))^{2} \phi(\tau, s(\tau) \xi) \longrightarrow G(\xi) \quad \text { as } \tau \rightarrow \infty,
$$

in a suitable sense to be determined. This was established in the particular cases $K(\xi, \eta)=2$ (constant) and $K(\xi, \eta)=\xi+\eta$ (linear) in $[18,19]$ in the sense of weak convergence, with explicit rates given in $[7,33]$. Convergence in stronger norms for the constant kernel was also found in [7]. There is also a theory of fat-tailed profiles, which represent the asymptotic behaviour of solutions with slowly decaying tails. We do not consider them in this work, and we refer 
the reader to [18] for explicitly solvable kernels, and to [26,28,34] for results on existence and uniqueness of fat-tailed self-similar profiles with infinite mass.

In this paper we are able to prove the scaling hypothesis in the regime of finite mass, with an explicit rate, for small bounded perturbations of the constant kernel. That is, we consider kernels of the type

$$
K=K_{\varepsilon}(\xi, \eta)=2+\varepsilon W(\xi, \eta),
$$

where $\varepsilon>0$ and the function $W:(0,+\infty) \times(0,+\infty) \rightarrow \mathbb{R}$ must be continuous, symmetric in $\xi, \eta$, satisfy the bound

$$
0 \leq W(\xi, \eta) \leq 1 \quad \text { for all } \xi, \eta>0,
$$

and be homogeneous of degree zero:

$$
W(\lambda \xi, \lambda \eta)=W(\xi, \eta) \quad \text { for all } \xi, \eta, \lambda>0 .
$$

Suitable examples of $W$ include

$$
W(\xi, \eta)=\Psi\left(\xi^{\alpha} \eta^{-\alpha}+\xi^{-\alpha} \eta^{\alpha}\right),
$$

where $\alpha \in \mathbb{R}$ and $\Psi:(0,+\infty) \rightarrow[-1,1]$ is any continuous function. Averages of functions of this type for different $\alpha$ are also examples of coefficients satisfying (1.3)-(1.5).

Remark 1.1. The choice of $K_{0}=2$ is made for convenience, since by simple scaling arguments one can consider perturbations of any constant kernel (see Section 2.2). Consequently, also the lower bound in (1.4) can be slightly weakened, i.e. our result also holds for perturbations which may change sign, satisfying $|W(x, y)| \leq 1$. In fact, replacing the constant kernel by $2-\|W\|_{L^{\infty}}$ and the perturbation by $\widetilde{W}=W+\|W\|_{L^{\infty}}$ the assumption (1.4) is satisfied.

We prove that for $\varepsilon$ small enough, solutions to (1.1) approach a unique, universal self-similar profile at an explicit algebraic rate (which becomes exponential when self-similar variables are considered; see below), in the sense of the $\|\cdot\|_{L_{k}^{1}}$ norm defined by

$$
\|f\|_{L_{k}^{1}}:=\int_{0}^{\infty}|f(x)|(1+x)^{k} \mathrm{~d} x .
$$

Our main result is summarised in the following theorem:

Theorem 1.2. Let $K=K_{\varepsilon}$ be a bounded perturbation of the constant kernel satisfying (1.3), (1.4) and (1.5).

1. There exists $\varepsilon_{1}>0$ such that for $0 \leq \varepsilon \leq \varepsilon_{1}$ there exists a unique self-similar profile $G_{\varepsilon}$ with unit mass.

2. Given $R>0$ and $k>1$, there exists $0<\varepsilon_{3} \leq \varepsilon_{1}$ (depending only on $R$ and $k$ ) and $M$ (depending only on $k$ ) such that for $0 \leq \varepsilon<\varepsilon_{3}$ any solution $\phi$ to the Smoluchowski equation (1.1) with nonnegative initial condition $\phi_{0}$ such that

$$
\int_{0}^{\infty} \xi \phi_{0}(\xi) \mathrm{d} \xi=1, \quad \int_{0}^{\infty}\left|\phi_{0}(\xi)-G_{\varepsilon}(\xi)\right|(1+\xi)^{k} \mathrm{~d} \xi \leq R
$$

satisfies

$$
\left\|f(t, \cdot)-G_{\varepsilon}\right\|_{L_{k}^{1}} \leq C e^{-\lambda_{\varepsilon} t}\left\|f_{0}-G_{\varepsilon}\right\|_{L_{k}^{1}} \quad \text { for all } t \geq 0,
$$


with $\lambda_{\varepsilon}:=\frac{1}{2}-M \varepsilon$, for some $C>0$ depending on $k$ and $R$, and where $f(t, x):=$ $e^{2 t} \phi\left(e^{t}-1, x e^{t}\right)$. Equivalently,

$$
\int_{0}^{\infty}\left|(\tau+1)^{2} \phi(\tau,(\tau+1) x)-G_{\varepsilon}(x)\right|(1+x)^{k} \mathrm{~d} x \leq C(1+\tau)^{-\lambda_{\varepsilon}}\left\|\phi_{0}-G_{\varepsilon}\right\|_{L_{k}^{1}}
$$

for all $\tau \geq 0$.

All constants appearing in this theorem can be explicitly estimated.

As far as we know, this is the first time the scaling hypothesis can be proved to hold for kernels which do not allow for an explicit solution of equation (1.1). Results for the so-called diagonal kernels were obtained by [17], and in this case the approach to self-similarity does not happen for all initial conditions. The part of our result on uniqueness of the profiles is not new: it has been proved for more general perturbations by Laplace transform methods in $[25,27,30]$ and via compactness arguments in $L^{1}$ in [35]. An improvement here is that we are able to give an explicit estimate of $\varepsilon_{0}$ in the case of bounded perturbations, and explicit estimates on the closeness of $G_{\varepsilon}$ to $G_{0}=e^{-x}$, the self-similar profile for the equation with $K=2$. For kernels $K$ with negative homogeneity degree it has been recently proved that self-similar profiles are unique [16].

The strategy to prove our results is a perturbation argument, carried out in a constructive way, using the fact that the case of constant coefficients is fairly well understood. This has been done for kinetic equations involving the Boltzmann operator in [5,20,21,23], but has not been done for coagulation-type equations as far as we know. Results on convergence to equilibrium for the Becker-Döring equation were developed in [4] using properties of the linearised operator, with techniques similar to those in Section 8. We need three main ingredients in order to complete our perturbation arguments:

1. First, we need a good global exponential convergence result for the constant coefficients case. Convergence without rate is known since [18], and in order to obtain rates one can use results in $[7,33]$. It turns out that a global convergence in an $L^{1}$ or $L^{2}$ space is more convenient for us, so we use a refined version of the results in [7]. These results are given in Section 2.7, with precise estimates on the dependence of the constants given in Appendix A.

2. One also needs results on the stability of self-similar profiles with respect to the perturbation; that is, we need to show that a profile $G_{\varepsilon}$ associated to the perturbed kernel $K_{\varepsilon}$ must be close to the unique profile $G_{0}$ for the constant coefficient case. In the case of perturbations by a bounded coagulation coefficient, it turns out that the perturbation of the operator is continuous in weighted $L^{1}$ norms, so we are led to work in these spaces. This kind of stability results was studied in [35], and we are able to give a new proof with explicit estimates in Section 3.

3. Finally, we need to show that the linearised equation around the self-similar profile for the constant case has a spectral gap. More importantly, we need to do this in a norm which allows us to complete the perturbation argument, so again we are forced to work in weighted $L^{1}$ norms. A spectral gap in these spaces is proved in Section 6, using results from Sections 4 and 5 . 
The paper is organised as follows: Section 2 gathers some preliminary results which are known or can be obtained almost directly from existing results. In Section 3 we give bounds on self-similar profiles (some of which are new) and show a quantitative stability result in weighted $L^{1}$ norms. The result itself is not new, but we give a new proof that makes it fully quantitative. Sections 4-6 study the linearised operator and show it has a spectral gap in the weighted $L^{1}$ spaces we need. Finally, Sections 7 and 8 use all of this to show uniqueness and exponential stability of self-similar profiles for small values of the perturbation parameter $\varepsilon$.

\section{Preliminaries}

\subsection{Self-similar change of variables}

By scaling arguments and mass conservation we obtain that in the case of kernels of homogeneity zero the function $s(\tau)$ in $(1.2)$ is given by $s(\tau)=(1+\tau)$, up to a time shift. Thus, plugging the self-similar ansatz

$$
\phi(\tau, \xi)=(1+\tau)^{-2} f\left(\log (1+\tau),(1+\tau)^{-1} \xi\right)
$$

into (1.1) we obtain

$$
\begin{aligned}
\partial_{t} f(t, x)=\frac{1}{2} \int_{0}^{x} K(x-y, y) f( & -y, t) f(t, y) \mathrm{d} y \\
& \quad-f(t, x) \int_{0}^{\infty} K(x, y) f(t, y) \mathrm{d} y+2 f(t, x)+x \partial_{x} f(t, x) .
\end{aligned}
$$

To simplify the notation, let us define the operator

$$
\mathcal{C}_{K}(f, f):=\frac{1}{2} \int_{0}^{x} K(x-y, y) f(x-y) f(y) \mathrm{d} y-f(x) \int_{0}^{\infty} K(x, y) f(y) \mathrm{d} y,
$$

which motivates the definition of the following symmetric bilinear form which will be useful later:

$$
\begin{aligned}
\mathcal{C}_{K}(g, h):=\frac{1}{2} \int_{0}^{x} K(x-y, y) & g(x-y) h(y) \mathrm{d} y \\
& \quad-\frac{1}{2} g(x) \int_{0}^{\infty} K(x, y) h(y) \mathrm{d} y-\frac{1}{2} h(x) \int_{0}^{\infty} K(x, y) g(y) \mathrm{d} y .
\end{aligned}
$$

When the kernel $K$ is $K_{\varepsilon}=2+\varepsilon W$, as it is almost always the case in this paper, we will write $\mathcal{C}_{K_{\varepsilon}} \equiv \mathcal{C}_{\varepsilon}$. We can then write equation (2.1) in an abbreviated form as

$$
\partial_{t} f=\mathcal{C}_{K}(f, f)+2 f+x \partial_{x} f
$$

We refer to this equation as the Smoluchowski equation in self-similarity variables, or simply the self-similar Smoluchowski equation.

To simplify the notation at some places (especially when the kernel $K$ is constant), we may also use the following notation $(f * g)(x)=\int_{0}^{x} f(x-y) g(y) \mathrm{d} y$ for the convolution. 


\subsection{Scale invariances}

We collect here some elementary properties about (2.1). It is well-known that (2.1) preserves the total mass $m_{1}(t):=\int_{0}^{\infty} x f(x, t) \mathrm{d} x$, i.e. $m_{1}(t) \equiv m_{1}(0)$, provided that the kernel $K$ grows at most linearly at infinity. Yet, for kernels with superlinear growth, a loss of total mass in finite time occurs which is known as gelation (e.g. [10]).

Furthermore, if $f$ is a solution to (2.1) with kernel $K$ one easily checks that for any $\alpha>0$ the function $g=\frac{1}{\alpha} f$ solves the self-similar Smoluchowski equation with kernel $\alpha K$, i.e.

$$
\partial_{t} g=\mathcal{C}_{\alpha K}(g, g)+2 g+x \partial_{x} g .
$$

Moreover, one verifies that for each solution $f$ of $(2.1)$ also the rescaled function $f_{a}:=a f(a x)$ is a solution to (2.1) with the same kernel. Note also that for both transformations the mass changes. Summarising, we find that for $f$ solving (2.1) with kernel $K$, the function $h(x)=\frac{a}{\alpha} f(a x)$ is a solution to (2.1) with $K$ replaced by $\alpha K$. Moreover, if $f$ has total mass $m_{1}$, i.e. $\int_{0}^{\infty} x f(x) \mathrm{d} x=m_{1}$ we get for $h$ that

$$
\int_{0}^{\infty} x h(x) \mathrm{d} x=\frac{a}{\alpha} \int_{0}^{\infty} x f(a x) \mathrm{d} x=\frac{1}{a \alpha} \int_{0}^{\infty} x f(x) \mathrm{d} x=\frac{m_{1}}{a \alpha} .
$$

These computations allow to transform solutions to (2.1) for different constant kernels and modify the total mass. As a consequence, we can assume without loss of generality in the following that the constant kernel $K$ is given by $K=2$ and the total mass of the solutions and profiles is given by one.

Remark 2.1. Note also that in [7] the kernel was chosen to be $K \equiv 1$. However, by the considerations above, all results can be easily rescaled to the case $K=2$ which is what we will always implicitly do during this work.

\section{$2.3 \quad$ Function spaces}

We collect in this section the function spaces and corresponding notation which we use throughout this work. If nothing else is stated, all functions live on the set $(0, \infty)$. First, for a general weight function $w:(0, \infty) \rightarrow(0, \infty)$ and $p \in[0, \infty)$ we define in the usual way the weighted $L^{p}$ space

$$
L^{p}(w):=\left\{\left.f \in L^{p}\left|\int_{0}^{\infty}\right| f(x)\right|^{p} w(x) \mathrm{d} x<\infty\right\}
$$

with norm $\|f\|_{L^{p}(w)}:=\left(\int_{0}^{\infty}|f(x)|^{p} w(x) \mathrm{d} x\right)^{1 / p}$. The most important case for this work will be the choice $p=1$ and $w(x)=(1+x)^{k}$ with $k \geq 1$ which gives

$$
L^{1}\left((1+x)^{k}\right):=\left\{f \in L^{1}\left|\int_{0}^{\infty}\right| f(x) \mid(1+x)^{k} \mathrm{~d} x<\infty\right\}
$$

with corresponding norm $\|f\|_{L^{1}\left((1+x)^{k}\right)}:=\int_{0}^{\infty}|f(x)|(1+x)^{k} \mathrm{~d} x$. To simplify the notation at some places, we might also write $L_{k}^{1}=L^{1}\left((1+x)^{k}\right)$ and we might use the abbreviations $\|\cdot\|_{k}=\|\cdot\|_{L_{k}^{1}}=\|\cdot\|_{L^{1}\left((1+x)^{k}\right)}$. For parts of this work, we also need several spaces with rather weak norms defined via the primitive.

In particular, we define the norm

$$
\|h\|_{W^{-1, \infty}}:=\sup _{x>0}\left|\int_{x}^{\infty} h(y) \mathrm{d} y\right| .
$$


Moreover, we introduce the (weighted) $L^{2}$ space $H^{-1}\left(\mathrm{e}^{\mu x}\right)$ which arose naturally in [7]. More precisely, for $\mu \geq 0$ we define the norm

$$
\|h\|_{H^{-1}\left(\mathrm{e}^{\mu x}\right)}^{2}=\int_{0}^{\infty}\left(D^{-1} h(y)\right)^{2} \mathrm{e}^{\mu y} \mathrm{~d} y .
$$

where $D^{-1} h(y)=\int_{y}^{\infty} h(x) \mathrm{d} x$ denotes the primitive. This norm originates from the following scalar product

$$
\langle g, h\rangle_{H^{-1}\left(\mathrm{e}^{\mu x}\right)}=\int_{0}^{\infty}\left(D^{-1} g\right)(x)\left(D^{-1} h\right)(x) \mathrm{e}^{\mu x} \mathrm{~d} x .
$$

The corresponding (Hilbert) space

$$
W^{-1,2}\left(\mathrm{e}^{\mu x}\right)=H^{-1}\left(\mathrm{e}^{\mu x}\right)
$$

is then given as the completion of $C_{c}^{\infty}(0, \infty)$ with respect to the norm $\|\cdot\|_{H^{-1}\left(\mathrm{e}^{\mu x}\right)}$. For $\mu>0$, we additionally introduce the corresponding subspace given by the constraint of zero first moment, i.e.

$$
H^{-1}\left(\mathrm{e}^{-\mu x}\right) \cap\left\{\int_{0}^{\infty} x h(x) \mathrm{d} x=0\right\}
$$

is defined as the completion of $\left(C_{c}^{\infty}(0, \infty) \cap\left\{\int_{0}^{\infty} x h(x) \mathrm{d} x=0\right\}\right)$ with respect to $\|\cdot\|_{H^{-1}\left(\mathrm{e}^{\mu x}\right)}$. Remark 2.2. The latter definition is justified by the following estimate which exploits integration by parts as well as Hölder's inequality: for $h \in C_{c}^{\infty}(0, \infty)$ we have

$$
\begin{aligned}
& \int_{0}^{\infty} x h(x) \mathrm{d} x=-\int_{0}^{\infty} x \partial_{x}\left(\int_{x}^{\infty} h(z) \mathrm{d} z\right) \mathrm{d} x=\int_{0}^{\infty} \mathrm{e}^{-\frac{\mu}{2} x} \mathrm{e}^{\frac{\mu}{2} x}\left(\int_{x}^{\infty} h(z) \mathrm{d} z\right) \mathrm{d} x \\
& \leq\left(\int_{0}^{\infty} \mathrm{e}^{-\mu x} \mathrm{~d} x\right)^{1 / 2}\|h\|_{H^{-1}\left(\mathrm{e}^{\mu x}\right)}=\mu^{-1 / 2}\|h\|_{H^{-1}\left(\mathrm{e}^{\mu x}\right)} .
\end{aligned}
$$

Thus, by density the integral $\int_{0}^{\infty} x h(x) \mathrm{d} x$ is meaningful for all $h \in H^{-1}\left(\mathrm{e}^{\mu x}\right)$.

We also note the following continuous embeddings which will be especially important for this work.

Lemma 2.3. For each $k \geq 0$ and $\mu>0$ the space $L^{2}\left(\mathrm{e}^{\mu x}\right)$ embeds continuously into $L^{1}((1+$ $\left.x)^{k}\right)$.

Proof. Using the splitting $1=\mathrm{e}^{-\mu x / 2} \mathrm{e}^{\mu x / 2}$ and Hölder's inequality we find

$$
\begin{gathered}
\|h\|_{L_{k}^{1}}=\int_{0}^{\infty}|h(x)|(1+x)^{k} \mathrm{~d} x=\int_{0}^{\infty}|h(x)| \mathrm{e}^{\frac{\mu}{2} x} \mathrm{e}^{-\frac{\mu}{2} x}(1+x)^{k} \mathrm{~d} x \\
\leq\left(\int_{0}^{\infty}|h(x)|^{2} \mathrm{e}^{\mu x} \mathrm{~d} x\right)^{1 / 2}\left(\int_{0}^{\infty} \mathrm{e}^{-\mu x}(1+x)^{2 k} \mathrm{~d} x\right)^{1 / 2} \leq C(\mu, k)\|h\|_{L^{2}\left(\mathrm{e}^{\mu x}\right)} .
\end{gathered}
$$

Lemma 2.4. For each $\mu>0$ the space $L^{2}\left(\mathrm{e}^{\mu x}\right)$ embeds continuously into $H^{-1}\left(\mathrm{e}^{\mu x}\right)$. 
Proof. It suffices to verify the embedding for the dense subset $C_{c}^{\infty}(0, \infty)$. In this case, integration by parts yields

$$
\begin{aligned}
\|h\|_{H^{-1}\left(\mathrm{e}^{\mu x}\right)}^{2}=\int_{0}^{\infty} \mathrm{e}^{\mu x}\left(\int_{x}^{\infty} h(z) \mathrm{d} z\right)^{2} \mathrm{~d} x=\frac{1}{\mu} \int_{0}^{\infty} \partial_{x}\left(\mathrm{e}^{\mu x}\right)\left(\int_{x}^{\infty} h(z) \mathrm{d} z\right)^{2} \mathrm{~d} x \\
=-\frac{1}{\mu}\left(\int_{0}^{\infty} h(z) \mathrm{d} z\right)^{2}+\frac{2}{\mu} \int_{0}^{\infty} \mathrm{e}^{\mu x}\left(\int_{x}^{\infty} h(z) \mathrm{d} z\right) h(x) \mathrm{d} x .
\end{aligned}
$$

Note, that we additionally exploit here that the boundary term at infinity vanishes since $h \in C_{c}^{\infty}$. To proceed, we use that the first expression on the right-hand side is non-positive while the second one can be estimated by means of Hölder's inequality together with the splitting $\mathrm{e}^{\mu x}=\mathrm{e}^{\frac{\mu}{2} x} \mathrm{e}^{\frac{\mu}{2} x}$ which results in

$$
\|h\|_{H^{-1}\left(\mathrm{e}^{\mu x}\right)}^{2} \leq \frac{2}{\mu}\|h\|_{H^{-1}\left(\mathrm{e}^{\mu x}\right)}\|h\|_{L^{2}\left(\mathrm{e}^{\mu x}\right)} .
$$

Cauchy's inequality with parameter $2 / \mu$ finally yields

$$
\|h\|_{H^{-1}\left(\mathrm{e}^{\mu x}\right)}^{2} \leq \frac{1}{2}\|h\|_{H^{-1}\left(\mathrm{e}^{\mu x}\right)}^{2}+\frac{2}{\mu^{2}}\|h\|_{L^{2}\left(\mathrm{e}^{\mu x}\right)}^{2}
$$

which finishes the proof by providing $\|h\|_{H^{-1}\left(\mathrm{e}^{\mu x}\right)}^{2} \leq 4 / \mu^{2}\|h\|_{L^{2}\left(\mathrm{e}^{\mu x}\right)}^{2}$.

\subsection{Continuity properties of $\mathcal{C}_{K}$}

The spaces $L^{1}\left((1+x)^{k}\right)$ are convenient to work with because, for a bounded kernel $K$, the coagulation operator $C_{K}$ is a continuous bilinear form on them, as we show in the next proposition. Notice that this is not true for weighted $L^{2}$ spaces, for example.

Proposition 2.5. For $K:(0, \infty)^{2} \rightarrow(0, \infty)$ bounded, the bilinear form $\mathcal{C}_{K}$ given by $(2.3)$ is continuous from $L^{1}\left((1+x)^{k}\right)$ to itself for each $k \geq 0$ and we have

$$
\left\|\mathcal{C}_{K}(g, h)\right\|_{L_{k}^{1}} \leq \frac{3}{2}\|K\|_{L^{\infty}}\|g\|_{L_{k}^{1}}\|h\|_{L_{k}^{1}} .
$$

Proof. From (2.3) together with Fubini's theorem we find

$$
\begin{aligned}
& \left\|\mathcal{C}_{K}(g, h)\right\|_{L_{k}^{1}} \leq \frac{1}{2} \int_{0}^{\infty} \int_{0}^{\infty}|K(x, y)||g(x)|+|h(y)|(1+(x+y))^{k} \mathrm{~d} x \mathrm{~d} y \\
+ & \frac{1}{2} \int_{0}^{\infty} \int_{0}^{\infty}|K(x, y)||g(x)||h(y)|(1+x)^{k} \mathrm{~d} x \mathrm{~d} y+\frac{1}{2} \int_{0}^{\infty} \int_{0}^{\infty}|K(x, y)||g(y)||h(x)|(1+x)^{k} \mathrm{~d} x \mathrm{~d} y .
\end{aligned}
$$

Exploiting that $(1+(x+y))^{k} \leq(1+x)^{k}(1+y)^{k}$ and $1 \leq(1+x)^{k}$ if $x, y \in(0, \infty)$ and $k \geq 0$, we further get

$$
\begin{aligned}
\left\|\mathcal{C}_{K}(g, h)\right\|_{L_{k}^{1}} \leq \frac{3}{2}\|K\|_{L^{\infty}}\left(|g(x)|(1+x)^{k} \mathrm{~d} x\right)\left(\int_{0}^{\infty}|h(y)|(1+y)^{k} \mathrm{~d} y\right) & =\frac{3}{2}\|K\|_{L^{\infty}}\|g\|_{L_{k}^{1}}\|h\|_{L_{k}^{1}} .
\end{aligned}
$$




\subsection{Existence of time-dependent solutions and moment estimates}

Following [11] we will use the following concept of (mild) solutions to (2.1).

Definition 2.6. For $k \leq 1$ let $f_{0} \in L_{k}^{1}$. A function $f \in C\left([0, \infty), L_{k}^{1}\right)$ is denoted a (mild) solution to $(2.1)$ if it satisfies

$$
f=S_{t} f_{0}+\int_{0}^{t} S_{t-s} \mathcal{C}_{K}(f, f)(s) \mathrm{d} s
$$

where $\left(S_{t}\right)_{t \geq 0}$ is the semigroup generated by the operator $h \mapsto 2 h+x h^{\prime}$, i.e. $\left(S_{t} h\right)(x)=$ $\mathrm{e}^{2 t} h\left(\mathrm{e}^{t} x\right)$.

Remark 2.7. According to [11], the following two solution concepts are equivalent to mild solutions if $f \in C\left([0, \infty), L_{k}^{1}\right)$ :

1. $f$ is a weak or distributional solution, i.e. it satisfies

$$
\int_{0}^{T} \int_{0}^{\infty}\left(f \partial_{t} \varphi+\mathcal{C}_{K}(f, f) \varphi-f\left(2 \varphi-\partial_{x}(x \varphi)\right) \mathrm{d} x \mathrm{~d} t=\int_{0}^{\infty} f_{0} \varphi(0, \cdot) \mathrm{d} x\right.
$$

for all $T>0$ and $\varphi \in C_{c}^{1}([0, T) \times(0, \infty))$.

2. $f$ is a renormalised solution, i.e. it satisfies

$$
\frac{\mathrm{d}}{\mathrm{d} t} \int_{0}^{\infty} \beta(f) \varphi \mathrm{d} x=\int_{0}^{\infty} \mathcal{C}_{K}(f, f) \beta^{\prime}(f) \varphi-f\left(2 \varphi-\partial_{x}(x \varphi) \mathrm{d} x\right.
$$

in the sense of distributions in $[0, \infty)$ for all $\beta \in C^{1}(\mathbb{R}) \cap W^{1, \infty}(\mathbb{R})$ and $\varphi \in C^{1}(0, \infty) \cap$ $L^{\infty}(0, \infty)$.

The self-similar profiles are then seen to be the stationary solutions to (2.1).

Definition 2.8. A function $G \in L_{1}^{1}$ is a self-similar profile of (1.1) if it is a stationary solution to $(2.1)$, i.e. it is a fixed-point for $(2.4)$.

Remark 2.9. Due to Remark 2.7 we see that $G$ is a self-similar if it is a weak stationary solution to (2.1), i.e. with left-hand side zero.

Remark 2.10. Note that when dealing with self-similar profiles frequently an integrated version of (the stationary) equation (2.1) is used, i.e. $x^{2} G(x)=\int_{0}^{x} \int_{x-y}^{\infty} y K(y, z) G(y) G(z) \mathrm{d} z \mathrm{~d} y$ (e.g. $[25,27,29])$. However, in view of [9, Lemma 2.11] together with Proposition 3.3 both formulations are equivalent.

The next proposition is a classical result which provides the existence of solutions to (2.1) under the assumption of a bounded coagulation coefficient. A proof can be found for example in [11, Lemma 2.8]

Proposition 2.11. Let $K$ be a bounded, symmetric and homogeneous of degree zero. For $k \geq 1$ let $f_{0} \in L^{1}\left((1+x)^{k}\right)$. Then there exists a unique solution $f \in L^{1}\left((1+x)^{k}\right)$ to (2.1) on $(0, \infty)$ with $f(0, \cdot)=f_{0}$. Moreover, there exists $C_{k}$, which only depends on $\left\|f_{0}\right\|_{L_{k}^{1}}$ increasingly, such that

$$
\sup _{t \geq 0}\|f(t, \cdot)\|_{L_{k}^{1}} \leq C_{k}
$$


Remark 2.12. Note that the bound on the norm provided by [11, Lemma 2.8] is local in time. However, arguing analogously as in the proof of Proposition 3.3 one can easily verify that the estimate holds globally as stated above.

The next statement provides a more explicit estimate on the integral of solutions to (2.1) for bounded perturbations of the constant kernel.

Proposition 2.13. Let $W$ satisfy (1.3)-(1.5) and let $f_{\varepsilon}$ be a solution to (2.1) with kernel $K_{\varepsilon}=2+\varepsilon W$ and denote $m_{0}(t):=\int_{0}^{\infty} f_{\varepsilon}(x, t) \mathrm{d} x$. Then the estimate

$$
m_{0}(t) \leq \frac{\mathrm{e}^{t}}{\frac{1}{m_{0}(0)}+\mathrm{e}^{t}-1} \leq \max \left\{m_{0}(0), 1\right\}
$$

holds for all $t \geq 0$.

Proof. It is well-known that $\mathcal{C}_{K}$ satisfies the relation

$$
\int_{0}^{\infty} \mathcal{C}_{K}(f, f)(x) \mathrm{d} x=-\frac{1}{2} \int_{0}^{\infty} \int_{0}^{\infty} K(x, y) f(x) f(y) \mathrm{d} x \mathrm{~d} y
$$

which follows from Fubini's Theorem. Thus, integrating (2.1) and noting that integration by parts yields $\int_{0}^{\infty} x f_{\varepsilon}^{\prime}(x, t) \mathrm{d} x=-m_{0}(t)$ we obtain

$$
\begin{aligned}
\partial_{t} m_{0}(t)=2 m_{0}(t) & -m_{0}(t)-\frac{1}{2} \int_{0}^{\infty} \int_{0}^{\infty} K_{\varepsilon}(x, y) f_{\varepsilon}(x) f_{\varepsilon}(y) \mathrm{d} x \mathrm{~d} y \\
& =m_{0}(t)-m_{0}^{2}(t)-\frac{\varepsilon}{2} \int_{0}^{\infty} \int_{0}^{\infty} W(x, y) f_{\varepsilon}(x) f_{\varepsilon}(y) \mathrm{d} x \mathrm{~d} y \leq m_{0}(t)-m_{0}^{2}(t) .
\end{aligned}
$$

In the last step, we exploited that $W, f_{\varepsilon} \geq 0$. Solving this differential inequality explicitly, the claim directly follows.

Remark 2.14. The result of the previous proposition holds in general for any kernel $K \geq 2$, as long as time-dependent solutions can be proved to exist for that kernel. We have stated it for $K=2+\varepsilon W$ since it is the only case used in the rest of this paper.

\subsection{Stability of time-dependent solutions with respect to perturbations}

Proposition 2.11 together with the continuity results in Proposition 2.5 easily implies that on a fixed time interval solutions to (2.1) for the perturbed and unperturbed kernel stay close at order $O(\varepsilon)$ :

Lemma 2.15. Let $K_{\varepsilon}$ satisfy (1.3)-(1.5) and let $f^{\varepsilon}$ and $f^{0}$ be the solutions to (2.1) with kernels $K_{\varepsilon}$ and $K_{0}$, respectively, and with the same initial condition $f^{\varepsilon}(0, \cdot)=f^{0}(0, \cdot)$. Then there exists a constant $C>0$ depending only on $\left\|f_{0}\right\|_{L_{k}^{1}}$ such that

$$
\left\|f^{\varepsilon}(t, \cdot)-f^{0}(t, \cdot)\right\|_{L_{k}^{1}} \leq \varepsilon C\left(\mathrm{e}^{C t}-1\right) .
$$

Also, the constant $C>0$ depends increasingly on $\left\|f_{0}\right\|_{L_{k}^{1}}$. 
Proof. We take the difference of (2.1) for $\varepsilon$ and $\varepsilon=0$ and multiply by $\operatorname{sgn}\left(f^{\varepsilon}-f^{0}\right)$ which allows to rewrite

$$
\begin{aligned}
\partial_{t}\left|f^{\varepsilon}(t, \cdot)-f^{0}(t, \cdot)\right|=\left(\mathcal{C}_{2+\varepsilon W}\left(f^{\varepsilon}, f^{\varepsilon}\right)-\mathcal{C}_{2}\left(f^{0}, f^{0}\right)\right) \operatorname{sgn}\left(f^{\varepsilon}-f^{0}\right) \\
+2\left|f^{\varepsilon}(t, \cdot)-f^{0}(t, \cdot)\right|+x \partial_{x}\left|f^{\varepsilon}(t, \cdot)-f^{0}(t, \cdot)\right| .
\end{aligned}
$$

We multiply by $(1+x)^{k}$, integrate over $(0, \infty)$ and use that integration by parts allows to rewrite and estimate

$$
\begin{aligned}
& \int_{0}^{\infty} x(1+x)^{k} \partial_{x}\left|f^{\varepsilon}(t, x)-f^{0}(t, x)\right| \mathrm{d} x \\
& =-\left\|f^{\varepsilon}(t, \cdot)-f^{0}(t, \cdot)\right\|_{L^{1}\left((1+x)^{k}\right.}-k \int_{0}^{\infty} x(1+x)^{k-1}\left|f^{\varepsilon}(t, x)-f^{0}(t, x)\right| \mathrm{d} x \\
& \leq-\left\|f^{\varepsilon}(t, \cdot)-f^{0}(t, \cdot)\right\|_{L_{k}^{1}} .
\end{aligned}
$$

Thus, we obtain

$$
\frac{\mathrm{d}}{\mathrm{dt}}\left\|f^{\varepsilon}-f^{0}\right\|_{L_{k}^{1}} \leq\left\|\mathcal{C}_{2+\varepsilon W}\left(f^{\varepsilon}, f^{\varepsilon}\right)-\mathcal{C}_{2}\left(f^{0}, f^{0}\right)\right\|_{L_{k}^{1}}+\left\|f^{\varepsilon}-f^{0}\right\|_{L_{k}^{1}}
$$

Notice that we have omitted the variables $(t, \cdot)$ for brevity. Using that $\mathcal{C}_{2+\varepsilon W}\left(f^{\varepsilon}, f^{\varepsilon}\right)-$ $\mathcal{C}_{2}\left(f^{0}, f^{0}\right)=\mathcal{C}_{2}\left(f^{\varepsilon}-f^{0}, f^{\varepsilon}+f^{0}\right)+\varepsilon \mathcal{C}_{W}\left(f^{\varepsilon}, f^{\varepsilon}\right)$ we can estimate this further, using Proposition 2.5:

$$
\begin{aligned}
\frac{\mathrm{d}}{\mathrm{dt}}\left\|f^{\varepsilon}-f^{0}\right\|_{L_{k}^{1}} \leq\left\|\mathcal{C}_{2}\left(f^{\varepsilon}-f^{0}, f^{\varepsilon}+f^{0}\right)\right\|_{L_{k}^{1}}+\varepsilon\left\|\mathcal{C}_{W}\left(f^{\varepsilon}, f^{\varepsilon}\right)\right\|_{L_{k}^{1}} \\
\quad \leq 3\left\|f^{\varepsilon}-f^{0}\right\|_{L_{k}^{1}}\left\|f^{\varepsilon}+f^{0}\right\|_{L_{k}^{1}}+\varepsilon\left\|f^{\varepsilon}\right\|_{L_{k}^{1}}^{2}
\end{aligned}
$$

From Proposition 2.11 we know that there exists $C_{1}>0$ depending only on $\left\|f^{0}(0, \cdot)\right\|_{L_{k}^{1}}$ (and increasingly) such that

$$
\left\|f^{\varepsilon}\right\|_{L_{k}^{1}}+\left\|f^{0}\right\|_{L_{k}^{1}} \leq C_{1} \quad \text { for all } t \geq 0
$$

Hence,

$$
\frac{\mathrm{d}}{\mathrm{dt}}\left\|f^{\varepsilon}-f^{0}\right\|_{L_{k}^{1}} \leq 3 C_{1}\left\|f^{\varepsilon}-f^{0}\right\|_{L_{k}^{1}}+\varepsilon C_{1}^{2} .
$$

From Gronwall's inequality we get

$$
\left\|f^{\varepsilon}-f^{0}\right\|_{L_{k}^{1}} \leq \frac{\varepsilon C_{1}}{3}\left(\mathrm{e}^{3 C_{1} t}-1\right),
$$

which implies the statement for $C:=3 C_{1}$.

\subsection{Asymptotic behaviour of solutions for the constant kernel}

First, we recall from [7, Lemma 6.1] the following statement which provides exponential convergence in $L^{2}$ to the unique profile for the constant coagulation kernel $K=2$. 
Theorem 2.16. Let $f$ be a solution to (2.1) for the constant kernel $K=2$ with total mass one and initial condition $f_{0}$ such that $f_{0} \in L^{2}(\mathrm{~d} x) \cap L^{1}\left(x^{2} \mathrm{~d} x\right)$. Let $G^{0}(x)=\mathrm{e}^{-x}$ be the unique stationary solution to (2.1) with total mass 1 . There exists an explicit constant $C>0$ depending only on $\left\|f_{0}\right\|_{L^{2}}$ and $\left\|f_{0}\right\|_{L_{2}^{1}}$ such that

$$
\left\|f^{0}(t, \cdot)-G^{0}\right\|_{L^{2}} \leq C \mathrm{e}^{-\frac{1}{2} t} \quad \text { for all } t \geq 0 .
$$

Also, the constant $C>0$ depends increasingly on $\left\|f_{0}\right\|_{L^{2}}$ and $\left\|f_{0}\right\|_{L_{2}^{1}}$.

The statement we give here is slightly different to that in [7, Lemma 6.1] in that we say one can find an explicit constant $C$ depending only on $\left\|f_{0}\right\|_{L^{2}}$ and $\left\|f_{0}\right\|_{L_{2}^{1}}$. Although this constant was not specified in [7], this can be seen from the proof of the lemma, which consists on explicit estimates on the Fourier transform. We give a justification of this in Appendix A.

The aim of the following two lemmas is to transfer the convergence in Lemma 2.16 to $L^{1}\left((1+x)^{k}\right)$. For this, we first prove an elementary interpolation inequality (Lemma 2.17) which then allows to extend the convergence in Lemma 2.16 to $L^{1}\left((1+x)^{k}\right)$ (Lemma 2.18).

Lemma 2.17. Let $k^{*}>k$ and $\alpha \in\left(0, \min \left\{\frac{2\left(k^{*}-k\right)}{1+2 k^{*}}, 1\right\}\right)$ be given and assume that $f \in L^{1}((1+$ $\left.x)^{k^{*}}\right) \cap L^{2}(0, \infty)$. There exists a constant $C$ which depends on $\alpha, k$ and $k^{*}$ such that

$$
\|f\|_{L_{k}^{1}} \leq C\|f\|_{L^{2}}^{\alpha}\|f\|_{L_{k^{*}}^{1}}^{1-\alpha} .
$$

Proof. The claim follows from a straightforward application of Hölder's inequality with the three exponents $p_{1}=p_{2}=2 / \alpha$ and $p_{3}=1 /(1-\alpha)$. In fact, we have

$$
\begin{gathered}
\|f\|_{L_{k}^{1}}=\int_{0}^{\infty}|f(x)|(1+x)^{k} \mathrm{~d} x \\
=\int_{0}^{\infty}|f(x)|^{\alpha}|f(x)|^{1-\alpha}(1+x)^{(1-\alpha) k^{*}}(1+x)^{k-(1-\alpha) k^{*}} \mathrm{~d} x \\
\quad \leq\|f\|_{L^{2}(0, \infty)}^{\alpha}\|f\|_{L^{1}\left((1+x)^{k^{*}}\right)}^{1-\alpha}\left(\int_{0}^{\infty}(1+x)^{\frac{2\left(k-(1-\alpha) k^{*}\right)}{\alpha}} \mathrm{d} x\right)^{\frac{\alpha}{2}} .
\end{gathered}
$$

Since $\alpha<2\left((1-\alpha) k^{*}-k\right)$, the remaining integral on the right-hand side can easily be computed as

$$
\left(\int_{0}^{\infty}(1+x)^{\frac{2\left(k-(1-\alpha) k^{*}\right)}{\alpha}} \mathrm{d} x\right)^{\frac{\alpha}{2}}=\left(\frac{\alpha}{2\left((1-\alpha) k^{*}-k\right)-\alpha}\right)^{\frac{\alpha}{2}}
$$

which finishes the proof.

Lemma 2.18. Let $k^{*}>k \geq 2$, and let $f$ be a solution to (2.1) for the constant kernel $K=2$ with total mass one and initial condition $f_{0} \in L^{2} \cap L_{k^{*}}^{1}$, and let $G^{0}(x)=\mathrm{e}^{-x}$ be the unique stationary solution to (2.1) with total mass one. There exist constants $C, \beta>0$ such that

$$
\left\|f(t, \cdot)-G^{0}\right\|_{L_{k}^{1}} \leq C \mathrm{e}^{-\beta t} \quad \text { for all } t \geq 0 .
$$

The constant $C>0$ depends only on $k, k^{*},\left\|f_{0}\right\|_{L^{2}}$, and $\left\|f_{0}\right\|_{L_{k^{*}}^{1}}$ (and depends increasingly on the latter two). The constant $\beta>0$ depends only on $k$ and $k^{*}$. 
Proof. Due to [11, Lemma 2.8] we have that $f \in L^{\infty}\left(0, T, L^{2}\right)$ for all $T>0$. Thus, from Lemma 2.17 we know that, with an appropriate choice of $\alpha \in(0,1)$ (depending on $k$ and $k^{*}$ ),

$$
\left\|f^{0}(t, \cdot)-G^{0}\right\|_{L_{k}^{1}} \leq C_{1}\left\|f^{0}(t, \cdot)-G^{0}\right\|_{L^{2}}^{\alpha}\left\|f^{0}(t, \cdot)-G^{0}\right\|_{L_{k^{*}}^{1}}^{1-\alpha},
$$

for some $C_{1}>0$ depending only on $k$ and $k^{*}$. Thus, Young's inequality (with a parameter $\delta$ ) for $p_{1}=1 / \alpha$ and $p_{2}=1 /(1-\alpha)$ yields

$$
\left\|f^{0}(t, \cdot)-G^{0}\right\|_{L_{k}^{1}} \leq C_{2} \delta^{\frac{\alpha-1}{\alpha}}\left\|f^{0}(t, \cdot)-G^{0}\right\|_{L^{2}}+C_{1} \delta\left\|f^{0}(t, \cdot)-G^{0}\right\|_{L_{k^{*}}^{1}},
$$

again for $C_{2}>0$ depending only on $k, k^{*}$. Using Proposition 2.11 and Theorem 2.16 we obtain

$$
\left\|f^{0}(t, \cdot)-G^{0}\right\|_{L_{k}^{1}} \leq C_{3} \delta^{\frac{\alpha-1}{\alpha}} e^{-\frac{1}{2} t}+\delta C_{2},
$$

for some $C_{3}>0$ depending only on $k, k^{*},\left\|f_{0}\right\|_{L^{2}}$ and $\left\|f_{0}\right\|_{L_{2}^{1}}$, and $C_{2}>0$ depending only on $k^{*}$ and $\left\|f_{0}\right\|_{L_{k^{*}}^{1}}$. Taking $\delta=\mathrm{e}^{-\alpha t / 2}$ we deduce that

$$
\left\|f^{0}(t, \cdot)-G^{0}\right\|_{L_{k}^{1}} \leq C_{3} \mathrm{e}^{-\frac{\alpha}{2} t}+C_{2} \mathrm{e}^{-\frac{\alpha}{2} t}=\left(C_{2}+C_{3}\right) \mathrm{e}^{-\frac{\alpha}{2} t} .
$$

In sum, the constants $C_{2}$ and $C_{3}$ depend on $k, k^{*},\left\|f_{0}\right\|_{L^{2}},\left\|f_{0}\right\|_{L_{2}^{1}}$ and $\left\|f_{0}\right\|_{L_{k^{*}}^{1}}$. Since $\left\|f_{0}\right\|_{L_{2}^{1}} \leq$ $\left\|f_{0}\right\|_{L_{k^{*}}^{1}}$ (and $C_{2}, C_{3}$ are increasing in $\left\|f_{0}\right\|_{L_{2}^{1}}$ ), one can always modify the constants to have them depend only on $k, k^{*},\left\|f_{0}\right\|_{L^{2}}$, and $\left\|f_{0}\right\|_{L_{k^{*}}^{1}}$. Taking $\beta:=\alpha / 2$ and $C:=C_{2}+C_{3}$, this shows the result.

\section{Bounds and stability of self-similar profiles}

We gather in this section some basic results on existence and bounds which apply in particular to the self-similar profiles for the perturbed equation. More importantly, we give some stability results showing that any self-similar profile $G_{\varepsilon}$ with mass one for the kernel $K_{\varepsilon}$ must be close to $G_{0}(x)=e^{-x}$, in distances given by suitable norms. In general, these stability results cannot be obtained from the linearisation methods in this paper, so we borrow them from elsewhere or prove them using different methods. (However, linearisation methods do give some results on local stability of profiles, assuming we are in a certain neighbourhood of the profile $G_{0}$; see Lemma 7.2.)

\subsection{Existence of self-similar profiles}

Existence of self-similar profiles for large classes of non-solvable kernels with power-law structure was shown in $[9,11,12]$, and precise results in the case of homogeneity zero are given in [29]. Except for the works mentioned in the introduction, uniqueness of scaling profiles in not known for most coagulation kernels. However, there is a number of works providing a priori regularity and asymptotics of self-similar solutions for small and large cluster sizes (e.g. $[6,13,29])$.

In our particular setting of homogeneity zero, we cite the following result from [29, Prop. 1.1] which provides existence of self-similar profiles with finite mass for the kernels we consider (see also Remark 2.10):

Proposition 3.1 (Existence of profiles). Let $K$ be homogeneous of degree zero and let $k_{0}, K_{0}>$ 0 and $\kappa \in(0,1]$ be constants such that $K(x, y) \leq K_{0}\left((x / y)^{\alpha}+(y / x)^{\alpha}\right)$ for all $x, y \in(0, \infty)$ with $\alpha \in[0,1)$ and $\min _{|x-y| \leq \kappa(x+y)} K(x, y) \geq k_{0}$. Then there exists a self-similar profile $G \in C(0, \infty) \cap L^{1}(x \mathrm{~d} x)$ of $(1.1)$. 


\subsection{Bounds on self-similar profiles}

In this subsection, we provide several a-priori estimates for self-similar profiles. More precisely, Proposition 3.2 provides precise upper and lower bounds for the integral of perturbed selfsimilar profiles while Proposition 3.3 states that self-similar profiles are uniformly bounded in the $L_{k}^{1}$ norm.

Proposition 3.2. Let $K_{\varepsilon}$ satisfy (1.3)-(1.5) and let $G_{\varepsilon}$ be a stationary solution of (2.1), i.e. a self-similar profile. Then we have

$$
\frac{1}{1+\frac{\varepsilon}{2}} \leq \int_{0}^{\infty} G_{\varepsilon}(x) \mathrm{d} x \leq 1
$$

for all $\varepsilon \geq 0$.

Proof. By assumption $G_{\varepsilon}$ satisfies (2.1) with left-hand side zero. Integrating this equation, we find

$$
\begin{aligned}
2 \int_{0}^{\infty} G_{\varepsilon}(x) \mathrm{d} x+\int_{0}^{\infty} x G_{\varepsilon}^{\prime}(x) \mathrm{d} x+\frac{1}{2} \int_{0}^{\infty} & \int_{0}^{x}(2+\varepsilon W(x-y, y)) G_{\varepsilon}(x-y) G_{\varepsilon}(y) \mathrm{d} y \mathrm{~d} x \\
& -\int_{0}^{\infty} \int_{0}^{\infty}\left(2+\varepsilon W(x, y) G_{\varepsilon}(x) G_{\varepsilon}(y) \mathrm{d} x \mathrm{~d} y=0 .\right.
\end{aligned}
$$

Denoting $m_{0}=\int_{0}^{\infty} G_{\varepsilon}(x) \mathrm{d} x$, integrating by parts in the second term on the left-hand side and using Fubini's Theorem for the two double integrals, this reduces to

$$
\begin{aligned}
& 0=2 m_{0}-m_{0}+m_{0}^{2}-2 m_{0}^{2}+\frac{\varepsilon}{2} \int_{0}^{\infty} \int_{0}^{\infty} W(x, y) G_{\varepsilon}(x) G_{\varepsilon}(y) \mathrm{d} x \mathrm{~d} y \\
&-\varepsilon \int_{0}^{\infty} \int_{0}^{\infty} W(x, y) G_{\varepsilon}(x) G_{\varepsilon}(y) \mathrm{d} x \mathrm{~d} y .
\end{aligned}
$$

Combining terms, we end up with

$$
m_{0}=m_{0}^{2}+\frac{\varepsilon}{2} \int_{0}^{\infty} \int_{0}^{\infty} W(x, y) G_{\varepsilon}(x) G_{\varepsilon}(y) \mathrm{d} x \mathrm{~d} y .
$$

Due to (1.4) and the non-negativity of $G_{\varepsilon}$ we have $0 \leq W(x, y) G_{\varepsilon}(x) G_{\varepsilon}(y) \mathrm{d} x \mathrm{~d} y \leq m_{0}^{2}$ which leads to

$$
m_{0}^{2} \leq m_{0} \leq\left(1+\frac{\varepsilon}{2}\right) m_{0}^{2}
$$

from which the claim directly follows.

Based on the previous proposition, we can also show the following statement which gives uniform boundedness of all non-negative moments for self-similar profiles. We also note that this result also follows from estimates in [29] but to be self-contained, we include the complete proof.

Proposition 3.3. Let $K_{\varepsilon}$ satisfy (1.3)-(1.5) and $k \geq 0$. Then there exists a uniform constant $C_{k}$ (depending only on $k$ ) such that

$$
\left\|G_{\varepsilon}\right\|_{L_{k}^{1}}=\int_{0}^{\infty} G_{\varepsilon}(x)(1+x)^{k} \mathrm{~d} x \leq C_{k}
$$

for all self-similar profiles $G_{\varepsilon}$ and all $\varepsilon \in[0,1]$. 
Proof. We note that $(1+x)^{k} \leq C\left(1+x^{k}\right)$ for some $C=C(k)>0$. According to Proposition 3.2 it thus suffices to show that $\int_{0}^{\infty} x^{k} G_{\varepsilon}(x) \mathrm{d} x \leq C_{k}$ for all $k \in \mathbb{N}$ with $k \geq 2$.

To see this, we will argue by induction. Precisely, for a fixed $k \in \mathbb{N}$ with $k \geq 2$ assume that the moments up to order $k-1$ are bounded uniformly, i.e. $\int_{0}^{\infty} x^{\ell} G_{\varepsilon}(x) \mathrm{d} x \leq C_{\ell}$ for all $\ell \leq k-1$. For $R>1$ let $\varphi_{R}$ be the linear continuation of $x \mapsto x^{k}$, i.e.

$$
\varphi_{R}(x)= \begin{cases}x^{k} & \text { if } x \leq R \\ k R^{k-1} x-(k-1) R^{k} & \text { if } x>R .\end{cases}
$$

Since by assumption $G_{\varepsilon}$ has finite moments of order zero and one, we can take $\varphi_{R}$ as test function in the weak formulation of self-similar profiles which yields

$$
\begin{aligned}
& \int_{0}^{\infty} G_{\varepsilon}(x)\left(\left(x \varphi_{R}(x)\right)^{\prime}-2 \varphi_{R}(x)\right) \\
&=\left(\frac{1}{2} \int_{0}^{\infty} K(x, y) G_{\varepsilon}(x) G_{\varepsilon}(y)\left[\varphi_{R}(x+y)-\varphi_{R}(x)-\varphi_{R}(y)\right] \mathrm{d} y\right) \mathrm{d} x .
\end{aligned}
$$

It is easy to check that

$$
\varphi_{R}(x+y)-\varphi_{R}(x)-\varphi_{R}(y) \leq \widehat{C}_{k}\left(x^{k-1} y+x y^{k-1}\right)
$$

for all $x, y>0$ independent of $R$. Moreover, a direct computation yields

$$
\left(x \varphi_{R}(x)\right)^{\prime}-2 \varphi_{R}(x)=\left\{\begin{array}{ll}
(k-1) x^{k} & \text { if } x \leq R \\
(k-1) R^{k} & \text { if } x>R .
\end{array} .\right.
$$

Thus, we deduce from (3.1) together with (1.3) and (1.4) that

$$
\begin{aligned}
(k-1) \int_{0}^{R} x^{k} G_{\varepsilon}(x) \mathrm{d} x+(k-1) R^{k-1} & \int_{R}^{\infty} G_{\varepsilon}(x) \mathrm{d} x \\
& \leq \frac{3}{2} \widehat{C}_{k} \int_{0}^{\infty} \int_{0}^{\infty} G_{\varepsilon}(x) G_{\varepsilon}(y)\left[x^{k-1} y+x y^{k-1}\right] \mathrm{d} x \mathrm{~d} y .
\end{aligned}
$$

Due to the non-negativity of $G_{\varepsilon}$ we get in particular the estimate

$$
(k-1) \int_{0}^{R} x^{k} G_{\varepsilon}(x) \mathrm{d} x \leq 3 \widehat{C}_{k}\left(\int_{0}^{\infty} x^{k-1} G_{\varepsilon}(x) \mathrm{d} x\right)\left(\int_{0}^{\infty} y G_{\varepsilon}(y) \mathrm{d} y\right)
$$

from which the claim follows since the right-hand side is uniformly bounded by the induction assumption.

\subsection{Behaviour of profiles close to zero and a uniform $L^{2}$ estimate}

The main goal of this subsection is to provide a uniform bound in $L^{2}$ for self-similar profiles (Proposition 3.8). The main task for this consists in deriving the behaviour of the self-similar profiles for small values of $x$ which will be done in the following sequence of lemmata. As a byproduct, we also obtain an a-priori estimate for certain negative moments, depending on the perturbation parameter $\varepsilon$ (Lemma 3.7).

The first lemma provides a lower integral bound on the profiles which shows that the self-similar solutions can not concentrate at zero. 
Lemma 3.4. Let $K_{\varepsilon}$ satisfy (1.3)-(1.5). For each $a_{*} \in(0,1)$ there exists a constant $c_{*}>0$ such that

$$
\int_{a_{*}}^{\infty} G_{\varepsilon}(x) \mathrm{d} x \geq c_{*}
$$

for all self-similar profiles $G_{\varepsilon}$ with $\int_{0}^{\infty} x G_{\varepsilon}(x) \mathrm{d} x=1$.

Proof. Splitting the integral we find together with Cauchy's inequality and Proposition 3.2 that

$$
\begin{array}{r}
1=\int_{0}^{\infty} x G_{\varepsilon}(x) \mathrm{d} x=\int_{0}^{a_{*}} x G_{\varepsilon}(x) \mathrm{d} x+ \\
\int_{a_{*}}^{\infty} x G_{\varepsilon}(x) \mathrm{d} x \leq a_{*} \int_{0}^{a_{*}} G_{\varepsilon}(x) \mathrm{d} x+\int_{a_{*}}^{\infty} x G_{\varepsilon}(x) \mathrm{d} x \\
\leq a_{*}+\left(\int_{a_{*}}^{\infty} x^{2} G_{\varepsilon}(x) \mathrm{d} x\right)^{1 / 2}\left(\int_{a_{*}}^{\infty} G_{\varepsilon}(x) \mathrm{d} x\right)^{1 / 2} .
\end{array}
$$

Together with Proposition 3.3 we find

$$
1-a_{*} \leq C_{2}^{1 / 2}\left(\int_{a_{*}}^{\infty} G_{\varepsilon}(x) \mathrm{d} x\right)^{1 / 2}
$$

where $C_{2}$ is a uniform bound on the second moment which is provided by Proposition 3.3. Thus, since $a_{*} \in(0,1)$ we conclude

$$
\int_{a_{*}}^{\infty} G_{\varepsilon}(x) \mathrm{d} x \geq \frac{\left(1-a_{*}\right)^{2}}{C_{2}}
$$

and the claim follows with $c_{*}=\left(1-a_{*}\right)^{2} / C_{2}$.

The next statement gives an estimate on the primitive for self-similar profiles close to zero.

Lemma 3.5. Let $K_{\varepsilon}$ satisfy (1.3)-(1.5). There exist constants $C_{*}>0$ and $\varepsilon_{*} \in(0,1)$ such that for $\varepsilon \in\left(0, \varepsilon_{*}\right)$ each self-similar profile $G_{\varepsilon}$ satisfies

$$
\int_{0}^{x} G_{\varepsilon}(y) \mathrm{d} y \leq C_{*} x^{1-\frac{2 \varepsilon}{2+\varepsilon}} \quad \text { for all } x \leq 1
$$

In particular, this implies $\int_{0}^{x} G_{\varepsilon}(y) \mathrm{d} y \leq C_{*} x^{1-\varepsilon}$.

Proof. To simplify the notation, we denote $P(x):=\int_{0}^{x} G_{\varepsilon}(y) \mathrm{d} y$. We first note that it suffices to prove the claim for $x \leq a_{*}$ with $a_{*} \in(0,1)$ fixed. In fact, for $x>a_{*}$ we obtain by means of Proposition 2.13 that $P(x) \leq 1 \leq x / a_{*}$ and thus the claimed estimate holds with $C_{*}=1 / a_{*}$.

To prove the statement for $x \leq a_{*}$, we integrate the stationary version of (2.1) for $K=K_{\varepsilon}$ over $[0, x]$ to obtain

$$
\begin{aligned}
2 P(x)+\int_{0}^{x} z G_{\varepsilon}^{\prime}(z) \mathrm{d} z+\frac{1}{2} \int_{0}^{x} \int_{0}^{z} K_{\varepsilon}(z-y, y) & G_{\varepsilon}(z-y) G_{\varepsilon}(y) \mathrm{d} y \mathrm{~d} z \\
& -\int_{0}^{x} \int_{0}^{\infty} K_{\varepsilon}(z, y) G_{\varepsilon}(y) G_{\varepsilon}(z) \mathrm{d} y \mathrm{~d} z=0 .
\end{aligned}
$$


Integration by parts in the first integral and applying Fubini's theorem together with the change of variables $z \mapsto z+y$ we find

$$
\begin{aligned}
2 P(x)+x P^{\prime}(x)-P(x)+\frac{1}{2} \int_{0}^{x} \int_{0}^{x-y} K_{\varepsilon}(z, y) G_{\varepsilon}(y) G_{\varepsilon}(z) \mathrm{d} z \mathrm{~d} y & \\
& -\int_{0}^{x} \int_{0}^{\infty} K_{\varepsilon}(z, y) G_{\varepsilon}(y) G_{\varepsilon}(z) \mathrm{d} y \mathrm{~d} z=0 .
\end{aligned}
$$

Inserting $K_{\varepsilon}=2+\varepsilon W$ and summarising, this simplifies to

$$
\begin{aligned}
(1- & 2 P(\infty)) P(x)+x P^{\prime}(x)+\left(P * G_{\varepsilon}\right)(x) \\
& +\varepsilon\left(\frac{1}{2} \int_{0}^{x} \int_{0}^{x-y} W(y, z) G_{\varepsilon}(y) G_{\varepsilon}(z) \mathrm{d} z \mathrm{~d} y-\int_{0}^{x} \int_{0}^{\infty} W(y, z) G_{\varepsilon}(y) G_{\varepsilon}(z) \mathrm{d} y \mathrm{~d} z\right)=0 .
\end{aligned}
$$

We estimate the left-hand side from above by noting that the expression in parentheses is non-positive since the domain of integration for the negative term is larger than the one for the positive (which in addition has a factor of $1 / 2$ ). Moreover, by monotonicity we have $\left(P * G_{\varepsilon}\right)(x) \leq P^{2}(x)$. Together this implies

$$
0 \leq(1-2 P(\infty)) P(x)+x P^{\prime}(x)+P^{2}(x) .
$$

Denoting $\alpha:=2 P(\infty)-1=2 \int_{0}^{\infty} G_{\varepsilon}(x) \mathrm{d} x-1$ this can be equivalently written as

$$
-\alpha P(x)+x P^{\prime}(x) \geq-P^{2}(x) .
$$

This differential inequality can be solved explicitly. In fact, using the integration factor $x^{-\alpha-1}$ we get

$$
\frac{\mathrm{d}}{\mathrm{d} x}\left(x^{-\alpha} P(x)\right)=x^{-\alpha-1}\left(-\alpha P(x)+x P^{\prime}(x)\right) \geq-x^{-\alpha-1} P^{2}(x)=-x^{\alpha-1}\left(x^{-\alpha} P(x)\right)^{2} .
$$

We note that $P$ is monotonically non-decreasing. Thus, if $P\left(a_{*}\right)=0$ the claim is trivial. We therefore assume $P\left(a_{*}\right)>0$ which allows to rewrite on an interval $\left(a, a_{*}\right)$ (note that we only have to consider the region where $P$ is non-zero):

$$
-\frac{\mathrm{d}}{\mathrm{d} x}\left(x^{-\alpha} P(x)\right)^{-1}=\frac{\mathrm{d}}{\mathrm{d} x}\left(x^{-\alpha} P(x)\right)\left(x^{-\alpha} P(x)\right)^{-2} \geq-x^{\alpha-1} .
$$

Integrating this inequality over $\left(x, a_{*}\right)$ we obtain

$$
-\frac{1}{P\left(a_{*}\right)}+\frac{x^{\alpha}}{P(x)} \geq-\frac{1}{\alpha}\left(a_{*}^{\alpha}-x^{\alpha}\right)
$$

or equivalently

$$
\frac{x^{\alpha}}{P(x)} \geq \frac{1}{P\left(a_{*}\right)}-\frac{a_{*}^{\alpha}}{\alpha}+\frac{x^{\alpha}}{\alpha} .
$$

The definitions of $\alpha$ and $P$ imply

$$
\frac{1}{P\left(a_{*}\right)}-\frac{a_{*}^{\alpha}}{\alpha}=\frac{1}{\alpha P\left(a_{*}\right)}\left(\alpha-a_{*}^{\alpha} P\left(a_{*}\right)\right)=\frac{1}{\alpha P\left(a_{*}\right)}\left(2 \int_{0}^{\infty} G_{\varepsilon}(x) \mathrm{d} x-1-a_{*}^{\alpha} \int_{0}^{a_{*}} G_{\varepsilon}(x) \mathrm{d} x\right) .
$$


Since $\alpha>0$ due to Proposition 2.13 and $a_{*} \in(0,1)$ we have $a_{*}^{\alpha} \leq 1$ which yields together with Lemma 3.4 and Proposition 3.2 that for $\varepsilon$ sufficiently small, we have

$$
\begin{aligned}
\frac{1}{P\left(a_{*}\right)}-\frac{a_{*}^{\alpha}}{\alpha} \geq \frac{1}{\alpha P\left(a_{*}\right)} & \left(2 \int_{0}^{\infty} G_{\varepsilon}(x) \mathrm{d} x-1-\int_{0}^{a_{*}} G_{\varepsilon}(x) \mathrm{d} x\right) \\
=\frac{1}{\alpha P\left(a_{*}\right)} & \left(\int_{a_{*}}^{\infty} G_{\varepsilon}(x) \mathrm{d} x+\int_{0}^{\infty} G_{\varepsilon}(x) \mathrm{d} x-1\right) \\
& \geq \frac{1}{\alpha P\left(a_{*}\right)}\left(c_{*}+\frac{1}{1+\frac{\varepsilon}{2}}-1\right)=\frac{1}{\alpha P\left(a_{*}\right)}\left(c_{*}-\frac{\varepsilon}{2+\varepsilon}\right) .
\end{aligned}
$$

Thus, if $\varepsilon$ is small enough, the right-hand side is strictly positive (note that $\alpha$ is strictly positive due to Proposition 3.2). With this, we deduce from (3.2) and (3.3) that

$$
P(x) \leq \frac{x^{\alpha}}{\frac{1}{P\left(a_{*}\right)}-\frac{a_{*}^{\alpha}}{\alpha}+\frac{x^{\alpha}}{\alpha}} \leq \frac{\alpha P\left(a_{*}\right)}{c_{*}-\frac{\varepsilon}{2+\varepsilon}} x^{\alpha}=\frac{1}{c_{*}-\frac{\varepsilon}{2+\varepsilon}}\left(2 \int_{0}^{\infty} G_{\varepsilon}(y) \mathrm{d} y-1\right) \int_{0}^{a_{*}} G_{\varepsilon}(y) \mathrm{d} y x^{\alpha} .
$$

Together with Proposition 3.2 and the non-negativity of $G_{\varepsilon}$ the right-hand side can be further estimated to get

$$
P(x) \leq \frac{1}{c_{*}-\frac{\varepsilon}{2+\varepsilon}} x^{\alpha} .
$$

Finally, we recall again Proposition 3.2 to deduce $\alpha=2 \int_{0}^{\infty} G_{\varepsilon}(x) \mathrm{d} x-1 \geq 2 /(1+\varepsilon / 2)-1 \geq$ $(2-\varepsilon) /(2+\varepsilon)$ from which the claimed estimate follows.

Based on the preparation above, we can now provide a pointwise estimate on the behaviour of self-similar profiles close to zero.

Lemma 3.6. Let $K_{\varepsilon}$ satisfy (1.3)-(1.5). There exist constants $C_{*}>0$ and $\varepsilon_{*}>0$ such that each self-similar profiles $G_{\varepsilon}$ satisfies

$$
G_{\varepsilon}(x) \leq C_{*} x^{-\frac{2 \varepsilon}{2+\varepsilon}} \leq C_{*} x^{-\varepsilon} \quad \text { for almost all } x \leq 1
$$

if $\varepsilon \leq \varepsilon_{*}$.

Proof. We recall from Remark 2.10 that $G_{\varepsilon}$ satisfies the equation

$$
G_{\varepsilon}(x)=\frac{1}{x^{2}} \int_{0}^{x} \int_{x-y}^{\infty} y K_{\varepsilon}(y, z) G_{\varepsilon}(y) G_{\varepsilon}(z) \mathrm{d} z \mathrm{~d} y .
$$

The assumptions (1.3) and (1.4) together with $\varepsilon \leq 1$ imply $K_{\varepsilon} \leq 3$ which together with the non-negativity of $G_{\varepsilon}$ yields

$$
G_{\varepsilon}(x) \leq \frac{3}{x^{2}} \int_{0}^{x} \int_{x-y}^{\infty} y G_{\varepsilon}(y) G_{\varepsilon}(z) \mathrm{d} z \mathrm{~d} y \leq \frac{3}{x} \int_{0}^{x} \int_{0}^{\infty} G_{\varepsilon}(y) G_{\varepsilon}(z) \mathrm{d} z \mathrm{~d} y .
$$

Together with Lemma 3.4 and Proposition 3.2 we thus conclude

$$
G_{\varepsilon}(x) \leq \frac{3}{x} \int_{0}^{x} G_{\varepsilon}(y) \mathrm{d} y \leq 3 C_{*} x^{-\frac{2 \varepsilon}{2+\varepsilon}} .
$$


The next lemma gives uniform estimates also for certain negative moments.

Lemma 3.7. Let $\varepsilon_{*} \in(0,1)$ and let $K_{\varepsilon}$ satisfy (1.3)-(1.5) with $0 \leq \varepsilon \leq \varepsilon_{*}$. For each $\alpha \in\left(\varepsilon_{*}-1, \infty\right)$ there exists a constant $C_{\alpha}$ such that each self-similar profile $G_{\varepsilon}$ satisfies

$$
\int_{0}^{\infty} x^{\alpha} G_{\varepsilon}(x) \mathrm{d} x \leq C_{\alpha}
$$

Proof. The statement is a direct consequence of Proposition 3.3 and Lemma 3.6.

The preparation above now allows us to obtain uniform estimates on the $L^{2}$ norm of selfsimilar profiles.

Proposition 3.8. Let $K_{\varepsilon}$ satisfy (1.3)-(1.5). There exist constants $C_{*}>0$ and $\varepsilon_{*}>0$ such that each self-similar profiles $G_{\varepsilon}$ satisfies

$$
\left\|G_{\varepsilon}\right\|_{L^{2}(1)}=\left(\int_{0}^{\infty}\left|G_{\varepsilon}(x)\right|^{2} \mathrm{~d} x\right)^{1 / 2} \leq C_{*}
$$

if $\varepsilon \leq \varepsilon_{*}$.

Proof. We recall from the proof of Lemma 3.6 that

$$
G_{\varepsilon}(x)=\frac{1}{x^{2}} \int_{0}^{x} \int_{x-y}^{\infty} y K_{\varepsilon}(y, z) G_{\varepsilon}(y) G_{\varepsilon}(z) \mathrm{d} z \mathrm{~d} y .
$$

Thus, multiplying by $G_{\varepsilon}$ and integrating, together with Fubini's Theorem, we deduce

$$
\left\|G_{\varepsilon}\right\|_{L^{2}(1)}^{2}=\int_{0}^{\infty}\left|G_{\varepsilon}(x)\right|^{2} \mathrm{~d} x=\int_{0}^{\infty} \int_{0}^{\infty} y K_{\varepsilon}(y, z) G_{\varepsilon}(y) G_{\varepsilon}(z) \int_{y}^{y+z} \frac{G_{\varepsilon}(x)}{x^{2}} \mathrm{~d} x \mathrm{~d} z \mathrm{~d} y .
$$

Due to (1.3) and (1.4) and $\varepsilon \leq 1$ we find

$$
\left\|G_{\varepsilon}\right\|_{L^{2}(1)}^{2} \leq 3 \int_{0}^{\infty} \int_{0}^{\infty} y G_{\varepsilon}(y) G_{\varepsilon}(z) \int_{y}^{\infty} \frac{G_{\varepsilon}(x)}{x^{2}} \mathrm{~d} x \mathrm{~d} z \mathrm{~d} y .
$$

Next, we note that Proposition 3.3 and Lemma 3.6 directly imply that $\int_{y}^{\infty} G_{\varepsilon}(x) / x^{2} \mathrm{~d} x \leq$ $C y^{-1-\varepsilon}$ for all $y>0$ (note that we could obtain a much better decay for $y>1$ ). Using this, we deduce

$$
\left\|G_{\varepsilon}\right\|_{L^{2}(1)}^{2} \leq 3 \int_{0}^{\infty} y^{-\varepsilon} G_{\varepsilon}(y) \mathrm{d} y \int_{0}^{\infty} G_{\varepsilon}(z) \mathrm{d} z .
$$

The claim then follows from Proposition 3.3 and Lemma 3.7 if $\varepsilon<1 / 2$.

\subsection{Stability of profiles}

Regarding stability, the following statement is a particular case of [35, Thm. 2.4] for bounded perturbations $W$.

Proposition 3.9 (Stability of profiles). Let $W$ be a bounded kernel satisfying (1.4) and (1.5). For $\varepsilon \geq 0$, denote $K_{\varepsilon}:=2+\varepsilon W$. For any $k \geq 0$ there exists a function $\delta=\delta(\varepsilon)$ depending only $k$ and $\varepsilon$, with $\delta(\varepsilon) \rightarrow 0$ as $\varepsilon \rightarrow 0$ such that any self-similar profile $G_{\varepsilon}$ with mass 1 of Smoluchowski's equation with kernel $K_{\varepsilon}$ satisfies

$$
\left\|G_{\varepsilon}-G_{0}\right\|_{L_{k}^{1}} \leq \delta(\varepsilon)
$$


This is a basic property we need in order to complete the proofs of our main results in Sections 7 and 8. Its only drawback is that the stability rate $\delta=\delta(\varepsilon)$ was obtained in [35] via compactness arguments, and hence one cannot give any constructive estimate on it. As a consequence, using Proposition 3.9 as given, the constants in our main result in Theorem 1.2 would become non-constructive: we would not be able to estimate the size of $\varepsilon_{1}$ or $\varepsilon_{3}$, even if we know there must be one satisfying the statement.

In order to improve this situation we give an alternative way to show Proposition 3.9, which yields an explicit estimate on the rate $\delta(\varepsilon)$.

The main idea to obtain constructive estimates is to use available quantitative information on the asymptotic behaviour of solutions to the self-similar equation $\partial_{t} f=C_{0}(f, f)+2 f+x \partial_{x} f$ with constant coefficients. In a broad sketch, if we know that

1. the equation with constant coefficients relaxes to equilibrium, with explicit rates, in a certain norm,

2. the dynamics of solutions depends continuously on the perturbation $\varepsilon$, in the same norm,

3. and the norm of any profile $G_{\varepsilon}$ is bounded by a uniform constant,

then we can conclude that any profile $G_{\varepsilon}$ must be close to $G_{0}$ for small $\varepsilon$, in the same norm we are considering. Point 2 seems to be the least problematic of the three, and we will use our Lemma 2.15. Let us see what is available regarding point 1. Since [18] it is known that solutions in the constant coefficients case converge to equilibrium, and a quantitative estimate of the rate at which this happens was given in [7], in several norms including $L^{2}$ and weighted $L^{2}$ norms. A clean statement with explicit constants was then given in [33, Theorem 1.1], for $\|\cdot\|_{W^{-1, \infty}}$. Since we want to show that $\left\|G_{0}-G_{\varepsilon}\right\|_{L_{k}^{1}}$ is small, we are forced to use the $L^{2}$ norm convergence result in [7] to fulfill point 1 since a simple interpolation then allows us to control the $L_{k}^{1}$ norm. We have stated this result in our Lemma 2.18. Notice that we are also restricted by point 3 , since uniform estimates of profiles are available in $L_{k}^{1}$, but not for example in $L^{\infty}$ or $W^{1,1}$. For point 3, we use uniform estimates of profiles in $L^{2}$ given in Proposition 3.8, which as far as we know were not available elsewhere.

These ideas give us a proof of Proposition 3.9 with an explicit $\delta(\varepsilon)$ :

Proof Proposition 3.9. Take any solution $f^{\varepsilon}$ to (2.1) with kernel $K=2+\varepsilon W$, and any solution $f^{0}$ to (2.1) with constant kernel $K=2$. We have

$$
\left\|f^{\varepsilon}(t, \cdot)-G^{0}\right\|_{L_{k}^{1}} \leq\left\|f^{\varepsilon}(t, \cdot)-f^{0}(t, \cdot)\right\|_{L_{k}^{1}}+\left\|f^{0}(t, \cdot)-G^{0}\right\|_{L_{k}^{1}} .
$$

Let $G^{\varepsilon}$ be any self-similar profile with mass one for the kernel $K_{\varepsilon}$, and choose the initial condition for both $f^{\varepsilon}$ and $f^{0}$ to be equal to $G^{\varepsilon}$. In particular, $f^{\varepsilon}$ is then equal to the constantin-time profile $G^{\varepsilon}$. From Lemma 2.15,

$$
\left\|f^{\varepsilon}(t, \cdot)-f^{0}(t, \cdot)\right\|_{L_{k}^{1}}=\left\|G^{\varepsilon}-f^{0}(t, \cdot)\right\|_{L_{k}^{1}} \leq \varepsilon C_{1} \mathrm{e}^{C_{1} t} .
$$

for some $C_{1}>0$ depending only on $\left\|G^{\varepsilon}\right\|_{L_{k}^{1}}$, in an increasing way. Since we know from Lemma 3.3 that $\left\|G^{\varepsilon}\right\|_{L_{k}^{1}}$ is uniformly bounded by a constant $C_{k}$ depending only on $k$, we conclude that the constant $C_{1}$ can be chosen to depend only on $k$ as well. On the other hand, Lemma 2.18 shows that

$$
\left\|f^{0}(t, \cdot)-G^{0}\right\|_{L_{k}^{1}} \leq C_{2} \mathrm{e}^{-\beta t}
$$


for some constant $C_{2}>0$ depending on $k,\left\|G^{\varepsilon}\right\|_{L^{2}}$ and $\left\|G^{\varepsilon}\right\|_{L_{k+1}^{1}}$ (for example; any moment larger than $k$ will do, not necessarily $k+1$ ). The constant $\beta>0$ depends only on $k$. In a similar way as before, since we know from Propositions 3.3 and 3.8 that these norms of $G^{\varepsilon}$ are uniformly bounded by constants that depend only on $k$ we conclude that the constant $C_{2}$ can be chosen to depend only on $k$. Using our last two estimates in (3.4),

$$
\left\|G^{\varepsilon}-G^{0}\right\|_{L_{k}^{1}}=\left\|f^{\varepsilon}(\cdot, t)-G^{0}\right\|_{L_{k}^{1}} \leq \varepsilon C_{1} \mathrm{e}^{C_{1} t}+C_{2} \mathrm{e}^{-\beta t}, \quad t \geq 0 .
$$

for each solution $f^{\varepsilon}$ to $(2.1)$ with $K=2+\varepsilon W$. Choosing $t=\log \left(C_{2} \beta /\left(\varepsilon C_{1}^{2}\right)\right) /\left(C_{1}+\beta\right)$ the claim follows with $\delta(\varepsilon)=\varepsilon^{\beta /\left(C_{1}+\beta\right)}$.

Remark 3.10. In Section 7.1 we will give a further improvement of Proposition 3.9, i.e. we will show that actually $\delta(\varepsilon)=\mathcal{O}(\varepsilon)$ as $\varepsilon \rightarrow 0$.

\section{The linearised operator for the constant kernel and semi- group theory}

In this section we will introduce the linearised coagulation operator $\mathcal{L}_{0}$ in self-similar variables. Precisely, if we linearise the stationary version of (2.1) around the profile $G_{0}(x)=\mathrm{e}^{-x}$ we get

$$
\mathcal{L}_{0}[h]=x \partial_{x} h+2\left(G_{0} * h\right)-2 G_{0} \int_{0}^{\infty} h(y) \mathrm{d} y .
$$

Since this expression contains a derivative with respect to $x$ which is not defined in the spaces we consider, $\mathcal{L}_{0}$ has to be understood as an unbounded operator. However, $\mathcal{L}_{0}$ is obviously well-defined on $C_{c}^{\infty}(0, \infty)$ by the following equivalent representation formulas:

Lemma 4.1. On the space $C_{c}^{\infty}(0, \infty)$ the operator $\mathcal{L}_{0}$ as given in (4.1) can be equivalently written as

$$
\mathcal{L}_{0}[h](x)=x h^{\prime}(x)+2 e^{-x} \int_{0}^{x} h(y)\left(e^{y}-1\right) \mathrm{d} y-2 e^{-x} \int_{x}^{\infty} h(y) \mathrm{d} y
$$

and

$$
\mathcal{L}_{0}[h](x)=x h^{\prime}(x)-2 H(x)+2 \int_{0}^{x} H(y) \mathrm{e}^{-(x-y)} \mathrm{d} y
$$

where $H(x)=\int_{x}^{\infty} h(z) \mathrm{d} z$.

Proof. The expression (4.2) is obvious by splitting and re-combining the two integrals. For (4.3) we rewrite $h(y)=-\partial_{y}\left(\int_{y}^{\infty} h(z) \mathrm{d} z\right)$ in the convolution expression of (4.1) and integrate by parts which yields

$$
\begin{aligned}
& \mathcal{L}_{0}[h](x)=x h^{\prime}(x)+2 \int_{0}^{x}-\partial_{y} \int_{y}^{\infty} h(z) \mathrm{d} z \mathrm{e}^{-(x-y)} \mathrm{d} y-2 \mathrm{e}^{-x} \int_{0}^{\infty} h(y) \mathrm{d} y \\
= & x h^{\prime}(x)-2 \int_{x}^{\infty} h(z) \mathrm{d} z+2 \int_{0}^{\infty} h(z) \mathrm{d} z \mathrm{e}^{-x}+2 \int_{0}^{x} \int_{y}^{\infty} h(z) \mathrm{d} z \mathrm{e}^{-(x-y)} \mathrm{d} y-2 \mathrm{e}^{-x} \int_{0}^{\infty} h(y) \mathrm{d} y \\
& =-2 H(x)+2 \int_{0}^{x} H(y) \mathrm{e}^{-(x-y)} \mathrm{d} y .
\end{aligned}
$$


The main point of this section is to prove that $\mathcal{L}_{0}$, defined on a suitable domain, generates a strongly continuous semigroup in the spaces in which we will be working later:

Theorem 4.2. There are semigroups defined on each of the spaces $L^{1}\left((1+x)^{k}\right)$ for all $k \geq 0$, $L^{2}\left(\mathrm{e}^{\mu x}\right)$ and $H^{-1}\left(\mathrm{e}^{\mu x}\right)$ for all $\mu \geq 0$, such that

1. their generators are all defined on $\mathcal{C}_{c}^{\infty}$, and they are equal to $\mathcal{L}_{0}$ on $\mathcal{C}_{c}^{\infty}$,

2. and $\mathcal{C}_{c}^{\infty}$ is a core for their generators (see Definition 4.3 below).

These semigroups can all be restricted to the corresponding spaces with mass zero, i.e. their intersections with $\left\{\int_{0}^{\infty} x f(x) \mathrm{d} x=0\right\}$

When talking about $\mathcal{L}_{0}$ on any of these spaces, it is implicit that we mean the generator of the corresponding semigroup (equivalently, the closure of $\mathcal{L}_{0}$, defined on $\mathcal{C}_{c}^{\infty}$, in the corresponding norm).

The rest of this section is devoted to the proof of Theorem 4.2. To simplify working with unbounded operators and in particular with the corresponding domains in the following we introduce the notion of a core which usually allows to restrict to dense subsets instead of the full domain of the operator. The following definition is taken from [8, Ch. I, Definition 1.6]:

Definition 4.3 (Core). For an unbounded operator $U: D(U) \subset X \rightarrow X$ a subspace $S \subset D(U)$ is denoted core of $U$ if $S$ is dense in $D(U)$ for the graph norm $\|h\|_{U}=\|h\|+\|U h\|$.

The next lemma is an extension of the Bounded Perturbation Theorem stating that the latter also preserves the core of an unbounded operator. Since this result seems not to be proved in [8], we present the short proof for completeness.

Lemma 4.4. Let $U: D(U) \subset X \rightarrow X$ be the generator of a strongly continuous semigroup $\mathrm{e}^{U t}$ with core $S \subset D(U)$. Furthermore, let $V: X \rightarrow X$ be bounded. Then $U+V$ is the generator of a strongly continuous semigroup $\mathrm{e}^{(U+V) t}$ on $X$ with domain $D(U)$ and core $S$.

Proof. According to the Bounded Perturbation Theorem, the operator $U+V$ is the generator of a strongly continuous semigroup on $X$ with domain $D(U)$. To see that $S$ is still a core, it suffices to prove that the graph norms of $U$ and $U+V$, i.e. $\|\cdot\|_{U}$ and $\|\cdot\|_{U+V}$ are equivalent. For this, we fix $\kappa>1+\|V\|$ which yields

$$
\begin{aligned}
\|h\|_{U+V}=\|h\|+\|(U+V) h\| \geq & \|h\|+\frac{1}{\kappa}\|(U+V) h\| \geq\|h\|+\frac{1}{\kappa}\|U h\|-\frac{1}{\kappa}\|V h\| \\
& \geq\left(1-\frac{\|V\|}{\kappa}\right)\|h\|+\frac{1}{\kappa}\|U h\| \geq \frac{1}{\kappa}(\|h\|+\|U h\|)=\frac{1}{\kappa}\|h\|_{U} .
\end{aligned}
$$

Conversely, we find

$$
\begin{aligned}
\|h\|_{U+V}=\|h\|+\|(U+V) h\| \leq\|h\|+\|U h\| & +\|V h\| \leq(1+\|V\|)\|h\|+\|U h\| \\
& \leq(1+\|V\|)(\|h\|+\|U h\|) \leq(1+\|V\|)\|h\|_{U} .
\end{aligned}
$$

This finishes the proof.

The following remark states more precisely how the action of linear operators on the space $H^{-1}\left(\mathrm{e}^{\mu x}\right)$ will be understood in the following. 
Remark 4.5 (Definition of linear operators on $\left.H^{-1}\right)$. Since $H^{-1}\left(\mathrm{e}^{\mu x}\right)$ is a rather weak space, it seems most appropriate to define linear operators on it via a density argument. Precisely, due to the definition of $H^{-1}\left(\mathrm{e}^{\mu x}\right)$, the elements of this space are represented by equivalence classes of Cauchy sequences with respect to $\|\cdot\|_{H^{-1}\left(\mathrm{e}^{\mu x}\right)}$. In particular, for each class, we can always find a representative sequence which is contained in $C_{c}^{\infty}(0, \infty)$. The approach then consists in defining a given linear operator on this space and extend it again by completion with respect to the norm on $H^{-1}\left(\mathrm{e}^{\mu x}\right)$. This procedure of course requires that the operator defined this way in fact maps to $H^{-1}\left(\mathrm{e}^{\mu x}\right)$ and that the definition is independent of the specific choice of a sequence. However, both properties are obviously satisfied if the operator $U$ to be defined this way satisfies

$$
\|U h\|_{H^{-1}\left(\mathrm{e}^{\mu x}\right)} \leq C\|h\|_{H^{-1}\left(\mathrm{e}^{\mu x}\right)} \quad \text { for all } h \in C_{c}^{\infty}(0, \infty),
$$

i.e. the restriction of $U$ to $C_{c}^{\infty}(0, \infty)$ is bounded. For the operators considered in this work, the latter property will typically be satisfied and in this case, we implicitly use the construction described before.

The following lemma provides that $h \mapsto x h^{\prime}(x)$, which appears in the linearised coagulation operator, is the generator of a strongly continuous semigroup in the spaces $L_{k}^{1}, L^{2}\left(\mathrm{e}^{\mu x}\right)$ and $H^{-1}\left(\mathrm{e}^{\mu x}\right)$.

Lemma 4.6. The family of operators $\left(T_{t}\right)_{t \geq 0}$ given by the formula $\left(T_{t} h\right)(x)=h\left(x \mathrm{e}^{t}\right)$ defines a strongly continuous semigroup on $L^{1}\left((1+x)^{k}\right)$ for all $k \geq 0$ as well as on $L^{2}\left(\mathrm{e}^{\mu x}\right)$ and $H^{-1}\left(\mathrm{e}^{\mu x}\right)$ for all $\mu \geq 0$. Moreover, this defines also a semigroup on the corresponding spaces restricted to total mass equal to zero, i.e. $L^{1}\left((1+x)^{k}\right) \cap\left\{\int_{0}^{\infty} x f(x) \mathrm{d} x=0\right\}$ for all $k \geq 0$ as well as $L^{2}\left(\mathrm{e}^{\mu x}\right) \cap\left\{\int_{0}^{\infty} x f(x) \mathrm{d} x=0\right\}$ and $H^{-1}\left(\mathrm{e}^{\mu x}\right) \cap\left\{\int_{0}^{\infty} x f(x) \mathrm{d} x=0\right\}$ for $\mu>0$. In all cases the generator $\mathcal{B}_{1}$ is given by $\mathcal{B}_{1} h=x \frac{\mathrm{d}}{\mathrm{d} x}: h \mapsto x h^{\prime}(x)$ (while, by abuse of notation, we use the same notation for the generator on different spaces) and the space $C_{c}^{\infty}(0, \infty)$ or $C_{c}^{\infty}(0, \infty) \cap\left\{\int_{0}^{\infty} x f(x) \mathrm{d} x=0\right\}$ respectively is a core.

Moreover, we have

$$
\begin{aligned}
\left\|T_{t} h\right\|_{L_{k}^{1}} & \leq\|h\|_{L_{k}^{1}} \mathrm{e}^{-t} & & \text { for all } h \in L_{k}^{1} \\
\left\|T_{t} h\right\|_{L^{2}\left(\mathrm{e}^{\mu x}\right)} & \leq\|h\|_{L^{2}\left(\mathrm{e}^{\mu x}\right)} \mathrm{e}^{-\frac{1}{2} t} & & \text { for all } h \in L^{2}\left(\mathrm{e}^{\mu x}\right) \\
\left\|T_{t} h\right\|_{H^{-1}\left(\mathrm{e}^{\mu x}\right)} & \leq\|h\|_{H^{-1}\left(\mathrm{e}^{\mu x}\right)} \mathrm{e}^{-\frac{3}{2} t} & & \text { for } \text { all } h \in H^{-1}\left(\mathrm{e}^{\mu x}\right)
\end{aligned}
$$

and all $t \geq 0$.

Since the semigroup is explicitly given, the proof is straightforward but for the sake of completeness, we include it in Appendix C.

Proof of Theorem 4.2. We just apply the bounded perturbation theorem to the semigroups given by Lemma 4.6, since the reader can check that the remaining terms in the definition of $\mathcal{L}_{0}$ are bounded operators (in the $L^{1}$ and $L^{2}$ cases one can choose expression (4.2) for this; in the $H^{-1}$ case one can choose expression (4.3)).

\section{Tools on the spectral gap of linear operators}

The following technique allows us to obtain spectral gaps in different spaces, once a spectral gap in some space has been proved. These ideas stem from classical perturbation theory of 
linear operators, with constructive estimates given in [24] and a general theory developed in [15]. The simple approach described here was already used in [4], and we describe it below.

\subsection{Spectral gap}

We often refer to our estimates on the decay of several semigroups as "spectral gap estimates". The main decay property that we are interested in is more precisely called hypodissipativity:

Definition 5.1 (Hypodissipative semigroup). Let $X$ be a Banach space and $\mathcal{A}: D(\mathcal{A}) \subset X \rightarrow$ $X$ the generator of a strongly continuous semigroup $\mathrm{e}^{\mathcal{A} t}$. We say that $\mathcal{A}$ is hypodissipative (or that the semigroup $\left(e^{\mathcal{A} t}\right)_{t \geq 0}$ is hypodissipative) if there exist constants $C \geq 1, \lambda>0$ such that

$$
\left\|\mathrm{e}^{\mathcal{A} t} h\right\|_{X} \leq C\|h\|_{X} \mathrm{e}^{-\lambda t}
$$

for all $h \in X$.

An operator $\mathcal{A}$ is usually called dissipative if the above definition holds with $C=1$. If $\mathcal{A}$ is the generator of a strongly continuous semigroup on a Banach space $X$, we say $\mathcal{A}$ has a spectral gap if its kernel is nonzero (i.e., there are equilibria of the evolution), and the corresponding semigroup is hypodissipative when restricted to a suitable subspace of $X$ which does not contain the kernel of $\mathcal{A}$ (usually the subspace perpendicular to the kernel in a suitable scalar product). Since we do not define this "suitable subspace" in general, every time we mention a spectral gap result it should be clear that we always refer to a specific decay property of the corresponding semigroup.

Of course, this is intimately related to the property that the spectrum of $\mathcal{A}$ consists of 0 , plus an additional set contained in $\{z \in \mathbb{C} \mid \Re(z) \leq-\lambda\}$, but there is not a simple equivalence without further decay properties of the resolvent (by the Hille-Yosida theorem). This is why we prefer to work only with estimates on the decay of the associated semigroups.

\subsection{Restriction of the spectral gap}

We state a result similar to [4, Theorem 3.1] or [22, Theorem 1.1], dealing with restriction of the spectral gap of a linear operator instead of extension. Strictly speaking, the results below allow us to transfer the hypodissipativity property between semigroups. In order to use them to transfer a spectral gap property, we will later apply them to the subspaces perpendicular to the equilibrium in a suitable sense.

Theorem 5.2. Consider two Banach spaces $\mathcal{Y} \subseteq \mathcal{Z}$ with corresponding norms $\mathcal{Y}$ and $\mathcal{Z}$ such that $\|h\|_{\mathcal{Z}} \leq C_{\mathcal{Y}}\|h\|_{\mathcal{Y}}$ for all $h \in \mathcal{Y}$ for some $C_{\mathcal{Y}}>0$. Let $L_{\mathcal{Y}}: D\left(L_{\mathcal{Y}}\right) \rightarrow \mathcal{Y}$ be an unbounded operator which extends to an unbounded operator $L_{\mathcal{Z}}: D\left(L_{\mathcal{Z}}\right) \rightarrow \mathcal{Z}$ be unbounded, i.e. $D\left(L_{\mathcal{Y}}\right) \subseteq D\left(L_{\mathcal{Z}}\right)$ and $\left.L_{\mathcal{Z}}\right|_{D\left(L_{\mathcal{Y}}\right)}=L_{\mathcal{Y}}$. Given that:

1. $L_{\mathcal{Z}}$ is the generator of a strongly continuous semigroup $\mathrm{e}^{L_{\mathcal{Z}} t}$ on $\mathcal{Z}$.

2. which satisfies

$$
\left\|\mathrm{e}^{L_{\mathcal{Z}} t} h\right\|_{\mathcal{Z}} \leq C_{1} \mathrm{e}^{-\lambda_{1} t}\|h\|_{\mathcal{Z}}, \quad h \in \mathcal{Z},:: t \geq 0
$$

with $C_{1}>0$ and $\lambda_{1} \in \mathbb{R}$.

3. $L_{\mathcal{Z}}=A+B$ with linear operators $A, B$ on $\mathcal{Z}$ which satisfy 
(a) $A: \mathcal{Z} \rightarrow \mathcal{Y}$ is continuous, i.e. $\|A h\|_{\mathcal{Y}} \leq C_{A}\|h\|_{\mathcal{Z}}$ for all $h \in \mathcal{Z}$ and $C_{A}>0$,

(b) $B$ is the generator of a strongly continuous semigroup $\mathrm{e}^{B t}$ on $\mathcal{Y}$ satisfying

$$
\left\|\mathrm{e}^{B t} h\right\|_{\mathcal{Y}} \leq C_{2} \mathrm{e}^{-\lambda_{2} t}\|h\|_{\mathcal{Y}}, \quad h \in \mathcal{Y},:: t \geq 0
$$

with $C_{2}>0$, and $\lambda_{2} \neq \lambda_{1}$.

the operator $L_{\mathcal{Z}}$ (and thus $L_{\mathcal{Y}}$ ) has also a spectral gap on $\mathcal{Y}$, i.e. it satisfies

$$
\left\|\mathrm{e}^{L_{\mathcal{Z}} t} h\right\|_{\mathcal{Y}} \leq C \mathrm{e}^{-\min \left\{\lambda_{1}, \lambda_{2}\right\} t}\|h\|_{\mathcal{Y}}, \quad h \in \mathcal{Y},:: t \geq 0
$$

for $C=C_{2}\left(1+\frac{C_{A} C_{1} C_{\mathcal{Y}}}{\left|\lambda_{2}-\lambda_{1}\right|}\right)$.

Proof. We can use Duhamel's formula to write

$$
\mathrm{e}^{L_{\mathcal{Z}} t} h=\mathrm{e}^{B t} h+\int_{0}^{t} \mathrm{e}^{B(t-s)}\left(A \mathrm{e}^{L_{\mathcal{Z}} s} h\right) \mathrm{d} s \quad \forall h \in \mathcal{Y},: t \geq 0 .
$$

Thus, for fixed $h \in \mathcal{Y}$ and $t \geq 0$, we have

$$
\begin{aligned}
&\left\|\mathrm{e}^{L_{\mathcal{Z}} t} h\right\|_{\mathcal{Y}} \leq\left\|\mathrm{e}^{B t} h\right\|_{\mathcal{Y}}+\int_{0}^{t}\left\|\mathrm{e}^{B(t-s)}\left(A \mathrm{e}^{L_{\mathcal{Z}} s} h\right)\right\|_{\mathcal{Y}} \mathrm{d} s \\
& \leq C_{2} e^{-\lambda_{2} t}\|h\|_{\mathcal{Y}}+C_{2} \int_{0}^{t} e^{-\lambda_{2}(t-s)}\left\|A \mathrm{e}^{L_{\mathcal{Z}} s} h\right\|_{\mathcal{Y}} \mathrm{d} s \\
& \leq C_{2} e^{-\lambda_{2} t}\|h\|_{\mathcal{Y}}+C_{2} C_{A} \int_{0}^{t} e^{-\lambda_{2}(t-s)}\left\|\mathrm{e}^{L_{\mathcal{Z}} s} h\right\|_{\mathcal{Z}} \mathrm{d} s \\
& \leq C_{2} e^{-\lambda_{2} t}\|h\|_{\mathcal{Y}}+C_{2} C_{A} C_{1}\|h\|_{\mathcal{Z}} \int_{0}^{t} e^{-\lambda_{2}(t-s)} e^{-\lambda_{1} s} \mathrm{~d} s \\
&= C_{2} e^{-\lambda_{2} t}\|h\|_{\mathcal{Y}}+C_{2} C_{A} C_{1}\|h\|_{\mathcal{Z}} \frac{1}{\lambda_{2}-\lambda_{1}}\left(e^{-\lambda_{1} t}-e^{-\lambda_{2} t}\right) \\
& \leq C_{2}\left(1+\frac{C_{A} C_{1} C_{\mathcal{Y}}}{\left|\lambda_{2}-\lambda_{1}\right|}\right) e^{-\min \left\{\lambda_{1}, \lambda_{2}\right\} t}\|h\|_{\mathcal{Y}} .
\end{aligned}
$$

This shows the result.

\subsection{Extension of the spectral gap}

For convenience we recall [4, Theorem 3.1] which allows to extend the spectral gap from one Banach space to a larger one.

Theorem 5.3. Consider two Banach spaces $\mathcal{Y} \subset \mathcal{Z}$ with corresponding norms $\|\cdot\|_{\mathcal{Y}}$ and $\|\cdot\|_{\mathcal{Z}}$ and such that $\|h\|_{\mathcal{Z}} \leq C_{\mathcal{Y}}\|h\|_{\mathcal{Y}}$ for all $h \in \mathcal{Y}$. Let $L_{\mathcal{Y}}: D\left(L_{\mathcal{Y}}\right) \rightarrow \mathcal{Y}$ be an unbounded operator which extends to an unbounded operator $L_{\mathcal{Z}}: D\left(L_{\mathcal{Z}}\right) \rightarrow \mathcal{Z}$, i.e. $D\left(L_{\mathcal{Y}}\right) \subset D\left(L_{\mathcal{Z}}\right)$ and $\left.L_{\mathcal{Z}}\right|_{D\left(L_{\mathcal{Y}}\right)}=L_{\mathcal{Y}}$. Given that

1. Ly is the generator of a strongly continuous semigroup $\mathrm{e}^{L \mathcal{Y} t}$ on $\mathcal{Y}$

2. which satisfies

$$
\left\|\mathrm{e}^{L \mathcal{Y} t} h\right\|_{\mathcal{Y}} \leq C_{1} \mathrm{e}^{-\lambda_{1} t}\|h\|_{\mathcal{Y}} \quad \text { for } h \in \mathcal{Y} \text { and } t \geq 0
$$

with $C_{1}>0$ and $\lambda_{1} \in \mathbb{R}$ 
3. $L_{\mathcal{Z}}=\mathcal{A}+\mathcal{B}$ with linear operators $\mathcal{A}, \mathcal{B}$ on $\mathcal{Z}$ which satisfy

(a) $\mathcal{A}: \mathcal{Z} \rightarrow \mathcal{Y}$ is continuous, i.e. $\|\mathcal{A} h\|_{\mathcal{Y}} \leq C_{\mathcal{A}}\|h\|_{\mathcal{Z}}$ for all $h \in \mathcal{Z}$ and $C_{\mathcal{A}}>0$,

(b) $\mathcal{B}$ is the generator of a strongly continuous semigroup $\mathrm{e}^{\mathcal{B} t}$ on $\mathcal{Z}$ satisfying

$$
\left\|\mathrm{e}^{\mathcal{B} t} h\right\|_{\mathcal{Z}} \leq C_{2} \mathrm{e}^{-\lambda_{2} t}\|h\|_{\mathcal{Z}} \quad \text { for all } h \in \mathcal{Z} \text { and } t \geq 0
$$

with $C_{2}>0$ and $\lambda_{2}>\lambda_{1}$

the operator $L_{\mathcal{Z}}$ is the generator of a strongly continuous semigroup $\mathrm{e}^{L_{\mathcal{Z}} t}$ on $\mathcal{Z}$ which extends $\mathrm{e}^{L \mathcal{Y} t}$ and satisfies

$$
\left\|\mathrm{e}^{L_{\mathcal{Z}} t} h\right\|_{\mathcal{Z}} \leq C \mathrm{e}^{-\lambda_{1} t}\|h\|_{\mathcal{Z}} \quad \text { for all } h \in \mathcal{Z} \text { and } t \geq 0
$$

with $C=C_{2}+C_{\mathcal{Y}} C_{1} C_{2} C_{\mathcal{A}}\left(\lambda_{2}-\lambda_{1}\right)^{-1}$.

\section{Spectral gap for the constant kernel in weighted $L^{1}$ spaces}

Our overall plan for the linearised coagulation operator consists in showing that we can restrict the known $H^{-1}\left(e^{\mu x}\right)$ spectral gap to the smaller Hilbert space $L^{2}\left(\mathrm{e}^{\mu x}\right)$ and from there extend to the space $L^{1}\left((1+x)^{k}\right)$ with sufficiently large $k>0$, using the techniques from the previous section. In this section, we will transfer the spectral gap for $\mathcal{L}_{0}$ which has been obtained in [7] for the class of spaces $H^{-1}\left(\mathrm{e}^{\mu x}\right)$ to the spaces of the form $L^{1}\left((1+x)^{k}\right)$. For this, we will rely on the restriction/extension methods recapitulated in Section 5.

In [7] a spectral gap for $\mathcal{L}_{0}$ was obtained in $H^{-1}\left(\mathrm{e}^{\mu x}\right)$. Precisely, we recall from [7, Prop. 3.11 \& Lem. 3.12] the following result:

Theorem 6.1. For any $\mu \in(0,1)$, the operator $\mathcal{L}_{0}$ as given by $(4.3)$ has a spectral gap of size 1 in $H^{-1}\left(\mathrm{e}^{\mu x}\right)$, that is: on this space it generates a strongly continuous semigroup $\mathrm{e}^{\mathcal{L}_{0} t}$ satisfying

$$
\left\|\mathrm{e}^{\mathcal{L}_{0} t} h\right\|_{H^{-1}\left(\mathrm{e}^{\mu x}\right)} \leq\|h\|_{H^{-1}\left(\mathrm{e}^{\mu x}\right)} \mathrm{e}^{-t} \quad \text { for } t \geq 0
$$

and all $h \in H^{-1}\left(\mathrm{e}^{\mu x}\right) \cap\left\{\int_{0}^{\infty} x h(x) \mathrm{d} x=0\right\}$.

Remark 6.2. In [7] the constant kernel was chosen to be $K \equiv 1$, but the above result is adapted to our choice $K \equiv 2$ which seems to be more common.

\subsection{Restriction of the spectral gap to weigthed $L^{2}$ spaces}

In this subsection, we will prove the following proposition which states that the spectral gap for $\mathcal{L}_{0}$ in $H^{-1}\left(\mathrm{e}^{\mu x}\right)$ can be restricted to the subspace $L^{2}\left(\mathrm{e}^{\mu x}\right)$.

Proposition 6.3. The operator $\mathcal{L}_{0}$ as given by (4.3) generates a strongly continuous semigroup $\mathrm{e}^{\mathcal{L}_{0} t}$ on $L^{2}\left(\mathrm{e}^{\mu x}\right)$ and for each $\lambda \in(-\infty, 1 / 2]$ there exists $C_{\lambda}>0$ such that

$$
\left\|\mathrm{e}^{\mathcal{L}_{0} t} h_{0}\right\|_{L^{2}\left(\mathrm{e}^{\mu x}\right)} \leq C_{\lambda} \mathrm{e}^{-\lambda t}\left\|h_{0}\right\|_{L^{2}\left(\mathrm{e}^{\mu x}\right)} \quad \text { for all } h_{0} \in L^{2}\left(\mathrm{e}^{\mu x}\right) \text { with } \int_{0}^{\infty} x h_{0}(x) \mathrm{d} x=0
$$

and all $t \geq 0$. 
Proof. The proof follows from an application of Theorem 5.2. For this, we choose $\mathcal{Z}=$ $H^{-1}\left(\mathrm{e}^{\mu x}\right) \cap\left\{\int_{0}^{\infty} x f(x) \mathrm{d} x=0\right\}$ and $\mathcal{Y}=L^{2}\left(\mathrm{e}^{\mu x}\right) \cap\left\{\int_{0}^{\infty} x f(x) \mathrm{d} x=0\right\}$. Moreover, $\mathcal{L}_{\mathcal{Y}}$ and $\mathcal{L}_{\mathcal{Z}}$ are both given as unbounded operators by the expression (4.3) on the respective spaces (see also Remark 6.4 below).

1. According to [7, Proposition 3.11] (see Theorem 6.1 above) the operator $\mathcal{L}_{\mathcal{Z}}$ generates a strongly continuous semigroup $\mathrm{e}^{\mathcal{L}_{\mathcal{Z}} t}$ on the space $H^{-1}\left(\mathrm{e}^{\mu x}\right) \cap\left\{\int_{0}^{\infty} x f(x) \mathrm{d} x=0\right\}$.

2. From [7, Lemma 3.12] (see also Theorem 6.1 above) we have that

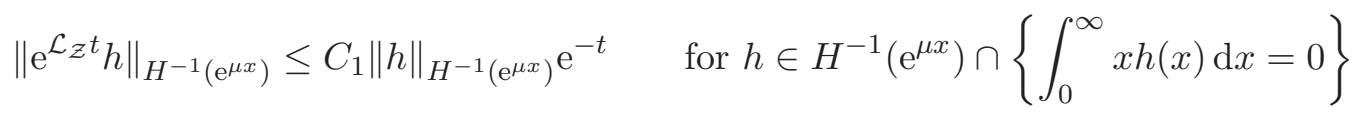

and all $t \geq 0$.

3. We split the operator $\mathcal{L}_{\mathcal{Z}}$ as follows: $\mathcal{L}_{\mathcal{Z}}=A+B$ with

$$
\begin{aligned}
& A[h](x)=-2 H(x)+2 \int_{0}^{x} H(y) \mathrm{e}^{-(x-y)} \mathrm{d} y=A_{1}[h](x)+A_{2}[h](x) \\
& B[h](x)=x \partial_{x} h(x) .
\end{aligned}
$$

Note that we use here the notation $H(x)=\int_{x}^{\infty} h(y) \mathrm{d} y$ for the primitive of $h$.

(a) We have to show that $A: \mathcal{Z} \rightarrow \mathcal{Y}$ is bounded. Since both $\mathcal{L}_{\mathcal{Z}}$ and $B$ preserve the constraint, the same is true for $A$ and thus it suffices to show that $A: H^{-1}\left(\mathrm{e}^{\mu x}\right) \rightarrow$ $L^{2}\left(\mathrm{e}^{\mu x}\right)$ is bounded. Obviously, we have $\left\|A_{1}[h]\right\|_{L^{2}\left(\mathrm{e}^{\mu x}\right)} \leq 2\|h\|_{H^{-1}\left(\mathrm{e}^{\mu x}\right)}$. Thus, it suffices to consider $A_{2}$ :

$$
\begin{aligned}
& \left\|A_{2}[h]\right\|_{L^{2}\left(\mathrm{e}^{\mu x}\right)}^{2}=4 \int_{0}^{\infty}\left(\int_{0}^{x} H(y) \mathrm{e}^{-(x-y)} \mathrm{d} y\right)^{2} \mathrm{e}^{\mu x} \mathrm{~d} x \\
& \quad=4 \int_{0}^{\infty}\left(\int_{0}^{x} H(y) \mathrm{e}^{\frac{\mu}{2} y} \mathrm{e}^{\left(\frac{\mu}{2}-1\right)(x-y)} \mathrm{d} y\right)^{2} \mathrm{~d} x=4\left\|\left(H(\cdot) \mathrm{e}^{\frac{\mu}{2} \cdot}\right) * \mathrm{e}^{\left(\frac{\mu}{2}-1\right)} \cdot\right\|_{L^{2}(\mathrm{~d} x)}^{2} .
\end{aligned}
$$

Here, $*$ denotes the convolution given by $(f * g)(x)=\int_{0}^{x} f(x-y) g(y) \mathrm{d} y$ for $x \in$ $(0, \infty)$. From Young's inequality for convolutions, we thus deduces

$$
\left\|A_{2}[h]\right\|_{L^{2}\left(\mathrm{e}^{\mu x}\right)} \leq 4\|h\|_{H^{-1}\left(\mathrm{e}^{\mu x}\right)}\left\|\mathrm{e}^{\left(\frac{\mu}{2}-1\right) \cdot}\right\|_{L^{1}(0, \infty)}=\frac{8}{2-\mu}\|h\|_{H^{-1}\left(\mathrm{e}^{\mu x}\right)} .
$$

(b) According to Lemma 4.6 the operator $B$ generates a strongly continuous semigroup $\mathrm{e}^{B t}$ on $L^{2}\left(\mathrm{e}^{\mu x}\right) \cap\left\{\int_{0}^{\infty} x f(x) \mathrm{d} x=0\right\}$ which satisfies

$$
\left\|\mathrm{e}^{B t} h\right\|_{L^{2}\left(\mathrm{e}^{\mu x}\right)} \leq\|h\|_{L^{2}\left(\mathrm{e}^{\mu x}\right)} \mathrm{e}^{-\frac{1}{2} t} \quad \text { for all } h \in L^{2}\left(\mathrm{e}^{\mu x}\right) \cap\left\{\int_{0}^{\infty} x f(x) \mathrm{d} x=0\right\}
$$

and $t \geq 0$.

Thus, according to Theorem 5.2 the claim follows.

Remark 6.4. To be able to use Theorem 5.2 in the previous proof, we have to make sure that $\mathcal{L}_{\mathcal{Z}}$ is an extension of $\mathcal{L}_{\mathcal{Y}}$. This follows from the following consideration: The proof of Proposition 6.3 (i.e. 3a) shows that $A$ is bounded from $\mathcal{Z}$ into $\mathcal{Y}$. According to Lemma 2.4 it is thus in particular bounded from $\mathcal{Z}$ into itself as well as from $\mathcal{Y}$ to itself. Lemmas 4.4 and 4.6 thus ensure that both $\mathcal{L}_{\mathcal{Z}}$ and $\mathcal{L}_{\mathcal{Y}}$ are generators of strongly continuous semigroups with common core $C_{c}^{\infty}(0, \infty)$ on which the two operators coincide. 


\subsection{Extension of the spectral gap to weighted $L^{1}$ spaces}

From Proposition 6.3 we know that $\mathcal{L}_{0}$ has a spectral gap in $L^{2}\left(\mathrm{e}^{\mu x}\right)$ subject to the constraint that $\int_{0}^{\infty} x f(x) \mathrm{d} x=0$. In this subsection we will now prove, using Theorem 5.3, that the latter can be extended to $L^{1}\left((1+x)^{k}\right)$. This is the main spectral gap result that will be used in the rest of this paper:

Theorem 6.5. Take $k>2$. The semigroup $e^{t \mathcal{L}_{0}}$ defined on the space $L^{1}\left((1+x)^{k}\right)$ (see 4.2) has a spectral gap, in the sense that there is $C=C(k)>0$ such that

$$
\left\|\mathrm{e}^{\mathcal{L}_{0} t} h_{0}\right\|_{k} \leq C \mathrm{e}^{-\frac{1}{2} t}\left\|h_{0}\right\|_{k} \quad \text { for all } t \geq 0
$$

for all $h_{0} \in L^{1}\left((1+x)^{k}\right)$ with $\int_{0}^{\infty} x h_{0}(x) \mathrm{d} x=0$. In particular, by the Hille-Yosida theorem we see that

$$
\left\|\mathcal{L}_{0}(h)\right\|_{L_{k}^{1}} \geq \frac{1}{2 C}\|h\|_{L_{k}^{1}}:=\frac{1}{M}\|h\|_{L_{k}^{1}}
$$

for all $h_{0}$ in the domain of $\mathcal{L}_{0}$ with $\int_{0}^{\infty} x h_{0}(x) \mathrm{d} x=0$

The proof of this statement will rely on an application of Theorem 5.3. Precisely, we choose $\mathcal{Y}=L^{2}\left(\mathrm{e}^{\mu x}\right)$ and $\mathcal{Z}=L^{1}\left((1+x)^{k}\right)$ and we will verify the following steps:

1. $\mathcal{L}_{0}$ given by (4.2) generates a strongly continuous semigroup $\mathrm{e}^{\mathcal{L}_{0} t}$ on $\mathcal{Y}$.

2. For each $\lambda_{1} \leq 1 / 2$, this semigroup satisfies $\left\|\mathrm{e}^{\mathcal{L}_{0} t} h\right\|_{L^{2}\left(\mathrm{e}^{\mu x}\right)} \leq C_{1} \mathrm{e}^{-\lambda_{1} t}\|h\|_{L^{2}\left(\mathrm{e}^{\mu x}\right)}$ for all $h \in L^{2}\left(\mathrm{e}^{\mu x}\right) \cap\left\{\int_{0}^{\infty} x h(x) \mathrm{d} x=0\right\}$ and $t \geq 0$.

3. There exists a splitting $\mathcal{L}_{0}=\mathcal{A}+\mathcal{B}$ such that:

(a) $\mathcal{A}: L_{k}^{1} \rightarrow L^{2}\left(\mathrm{e}^{\mu x}\right)$ is continuous, i.e. $\|\mathcal{A} h\|_{L^{2}\left(\mathrm{e}^{\mu x}\right)} \lesssim\|h\|_{L_{k}^{1}}$ for all $h \in L_{k}^{1}$ and $\int_{0}^{\infty} x \mathcal{A}[h](x) \mathrm{d} x=0$ for all $h \in L_{k}^{1}$ with $\int_{0}^{\infty} x h(x) \mathrm{d} x=0$.

(b) $\mathcal{B}$ generates a strongly continuous -semigroup $\mathrm{e}^{\mathcal{B} t}$ on $L_{k}^{1}$ satisfying

$$
\left\|\mathrm{e}^{\mathcal{B} t} h_{0}\right\|_{L_{k}^{1}} \leq C_{3} \mathrm{e}^{-t}\left\|h_{0}\right\|_{L_{k}^{1}} \text { for all } h_{0} \in L_{k}^{1} \text { with } \int_{0}^{\infty} x h_{0}(x) \mathrm{d} x=0 .
$$

In order to simplify the structure of the actual proof, we collect first several preparatory results while the proof of Theorem 6.5 will then be given at the end of this section.

We will choose the following splitting $\mathcal{L}_{0}=\mathcal{A}+\mathcal{B}$ of the operator $\mathcal{L}_{0}$ with

$$
\begin{aligned}
\mathcal{A}[h](x)=2 \mathrm{e}^{-x} \int_{0}^{x} h(y) \chi_{\{y \leq R\}}\left(\mathrm{e}^{y}-1\right) \mathrm{d} y-2 \mathrm{e}^{-x} \int_{x}^{\infty} h(y) \mathrm{d} y \\
\quad+\int_{0}^{\infty} 2 z \mathrm{e}^{-z} \int_{0}^{z} h(y) \chi_{\{y>R\}}\left(\mathrm{e}^{y}-1\right) \mathrm{d} y \mathrm{~d} z \mathrm{e}^{-x}=\mathcal{A}_{1}+\mathcal{A}_{2}+\mathcal{A}_{3} \\
\mathcal{B}[h](x)=x h^{\prime}(x)+2 \mathrm{e}^{-x} \int_{0}^{x} h(y) \chi_{\{y>R\}}\left(\mathrm{e}^{y}-1\right) \mathrm{d} y \\
\quad-\int_{0}^{\infty} 2 z \mathrm{e}^{-z} \int_{0}^{z} h(y) \chi_{\{y>R\}}\left(\mathrm{e}^{y}-1\right) \mathrm{d} y \mathrm{~d} z \mathrm{e}^{-x}=\mathcal{B}_{1}+\mathcal{B}_{2}+\mathcal{B}_{3}
\end{aligned}
$$

where $R$ is a sufficiently large constant which has to be fixed in the proof of Theorem 6.5 below. The last expression on the right-hand side of $\mathcal{A}$ and $\mathcal{B}$ ensures that $\int_{0}^{\infty} x \mathcal{A}[h](x) \mathrm{d} x=$ 
$0=\int_{0}^{\infty} x \mathcal{B}[h](x) \mathrm{d} x$. At this point, we also exploit that $\mathcal{L}_{0}$ preserves this constraint, i.e. we construct $\mathcal{B}$ such that the first moment is zero which implies that the same is automatically true for $\mathcal{A}$ since $\mathcal{L}_{0}=\mathcal{A}+\mathcal{B}$.

The following three lemmata provide estimates on auxiliary integrals which will turn out to be useful during subsequent computations.

Lemma 6.6. Let $k \in \mathbb{R}$. For each $\beta>1$ there exists $R_{\beta}>0$ such that

$$
\int_{y}^{\infty} \mathrm{e}^{-x}(1+x)^{k} \mathrm{~d} x \leq \beta \mathrm{e}^{-y}(1+y)^{k}
$$

if $y \geq R_{\beta}$.

Proof. An application of l'Hôpital's rule yields $\int_{y}^{\infty} \mathrm{e}^{-x}(1+x)^{k} \mathrm{~d} x \sim \mathrm{e}^{-y}(1+y)^{k}$ as $y \rightarrow \infty$ from which the claim directly follows.

Lemma 6.7. For each $k \geq 0$ we have the estimate

$$
\int_{0}^{\infty}(x+1)^{k} \mathrm{e}^{-x} \mathrm{~d} x \leq \frac{\Gamma(k+1)}{\mathrm{e}} .
$$

Proof. The definition of $\Gamma(\cdot)$ together with the change of variables $x \mapsto x-1$ yields

$$
\int_{0}^{\infty}(x+1)^{k} \mathrm{e}^{-x} \mathrm{~d} x=\int_{1}^{\infty} x^{k} \mathrm{e}^{-x-1} \mathrm{~d} x=\frac{1}{\mathrm{e}} \int_{1}^{\infty} x^{(k+1)-1} \mathrm{e}^{-x} \mathrm{~d} x \leq \frac{\Gamma(k+1)}{\mathrm{e}} .
$$

Lemma 6.8. For each $k \geq 0$ there exists a constant $C_{k}>0$ such that

$$
\int_{y}^{\infty} \mathrm{e}^{-x}(1+x)^{k} \mathrm{~d} x \leq C_{k} \mathrm{e}^{-y}(1+y)^{k}
$$

for all $y>0$.

Proof. Due to Lemma 6.6 there exists $R_{2}>0$ such that $\int_{y}^{\infty} \mathrm{e}^{-x}(1+x)^{k} \mathrm{~d} x \leq 2 \mathrm{e}^{-y}(1+y)^{k}$ if $y \geq R_{2}$. Moreover, if $y \leq R_{2}$ we deduce together with Lemma 6.7 that

$$
\int_{y}^{\infty} \mathrm{e}^{-x}(1+x)^{k} \mathrm{~d} x \leq \frac{\Gamma(k+1)}{\mathrm{e}} \leq \frac{\Gamma(k+1)}{\mathrm{e}} \mathrm{e}^{R_{2}}(1+y)^{k} \mathrm{e}^{-y}
$$

Combining both estimates, the claim follows with $C_{k}=\max \left\{2, \Gamma(k+1) \mathrm{e}^{R_{2}} / \mathrm{e}\right\}$.

The next lemma shows that the operators $\mathcal{B}_{2}$ and $\mathcal{B}_{3}$ are bounded.

Lemma 6.9. For any $\beta>1$ there exists $R_{\beta}>0$ such that the operators $\mathcal{B}_{2}, \mathcal{B}_{3}: L^{1}\left((1+x)^{k}\right) \rightarrow$ $L^{1}\left((1+x)^{k}\right)$ as defined in (6.1) are bounded with

$$
\left\|\mathcal{B}_{2} h\right\|_{L_{k}^{1}} \leq 2 \beta\|h\|_{L_{k}^{1}} \quad \text { and } \quad\left\|\mathcal{B}_{3} h\right\|_{L_{k}^{1}} \leq \frac{2 \Gamma(k+1)}{\mathrm{e}(R+1)^{k-1}}\|h\|_{L_{k}^{1}}
$$

if $R>R_{\beta}$. Moreover, there exists $C_{k}>0$ such that $\left\|\mathcal{B}_{2} h\right\|_{L_{k}^{1}} \leq C_{k}\|h\|_{L_{k}^{1}}$ for all $R \geq 0$. 
Proof. We first consider the correction term $\mathcal{A}_{3}=-\mathcal{B}_{3}$ given in (6.1) which we rewrite by means of Fubini's theorem and the relation $\int_{y}^{\infty} z \mathrm{e}^{-z} \mathrm{~d} z=(y+1) \mathrm{e}^{-y}$ which yields

$$
\begin{aligned}
\int_{0}^{\infty} 2 z \mathrm{e}^{-z} \int_{0}^{z} h(y) \chi_{\{y>R\}}\left(\mathrm{e}^{y}-1\right) \mathrm{d} y \mathrm{~d} z=2 \int_{0}^{\infty} h(y) \chi_{\{y>R\}}\left(\mathrm{e}^{y}-1\right) \int_{y}^{\infty} z \mathrm{e}^{-z} \mathrm{~d} z \mathrm{~d} y \\
=2 \int_{R}^{\infty} h(y)(y+1)\left(1-\mathrm{e}^{-y}\right) \mathrm{d} y=2 \int_{R}^{\infty} h(y)(y+1)\left(1-\mathrm{e}^{-y}\right) \mathrm{d} y .
\end{aligned}
$$

From this, we deduce in particular the estimate

$$
\begin{array}{r}
\left|\int_{0}^{\infty} 2 z \mathrm{e}^{-z} \int_{0}^{z} h(y) \chi_{\{y>R\}}\left(\mathrm{e}^{y}-1\right) \mathrm{d} y \mathrm{~d} z\right| \leq 2 \int_{R}^{\infty}|h(y)|(y+1)^{k}(y+1)^{1-k}\left(1-\mathrm{e}^{-y}\right) \mathrm{d} y \\
\leq 2(R+1)^{1-k} \int_{R}^{\infty}|h(y)|(y+1)^{k} \mathrm{~d} y \leq 2(R+1)^{1-k}\|h\|_{L_{k}^{1}}
\end{array}
$$

Thus, together with (6.1) and Lemma 6.7 we immediately get

$$
\left\|\mathcal{B}_{3} h\right\|_{L_{k}^{1}} \leq \frac{2 \Gamma(k+1)}{\mathrm{e}(R+1)^{k-1}}\|h\|_{L_{k}^{1}} .
$$

To bound $\mathcal{B}_{2}$ we note that by means of Fubini's theorem we have

$$
\begin{aligned}
\left\|\mathcal{B}_{2} h\right\|_{L_{k}^{1}} \leq 2 \int_{0}^{\infty} \mathrm{e}^{-x} \int_{0}^{x}|h(y)| \chi_{\{y>R\}}\left(\mathrm{e}^{y}-1\right) \mathrm{d} y(1+x)^{k} \mathrm{~d} x & \\
& =2 \int_{R}^{\infty}|h(y)|\left(\mathrm{e}^{y}-1\right) \int_{y}^{\infty} \mathrm{e}^{-x}(1+x)^{k} \mathrm{~d} x \mathrm{~d} y .
\end{aligned}
$$

Thus, on the one hand fixing $\beta>1$ we obtain together with Lemma 6.6 that

$$
\left\|\mathcal{B}_{2} h\right\|_{L_{k}^{1}} \leq 2 \beta \int_{R}^{\infty}|h(y)|(1+y)^{k}\left(1-\mathrm{e}^{-y}\right) \mathrm{d} y \leq 2 \beta\|h\|_{L_{k}^{1}} .
$$

if $R>R_{\beta}$. On the other hand, Lemma 6.8 yields

$$
\left\|\mathcal{B}_{2} h\right\|_{L_{k}^{1}} \leq C_{k} \int_{0}^{\infty}|h(y)|(1+y)^{k}\left(1-\mathrm{e}^{-y}\right) \mathrm{d} y \leq C_{k}\|h\|_{L_{k}^{1}}
$$

We next prove that the operator $\mathcal{B}$ generates a strongly continuous semigroup (which is the first part of Item $3 \mathrm{~b}$ above).

Lemma 6.10. Under the assumptions of Theorem 6.5 the operator $\mathcal{B}$ as defined in (6.1) generates a strongly continuous semigroup both on $L_{k}^{1}$ and $L_{k}^{1} \cap\left\{\int_{0}^{\infty} x f(x) \mathrm{d} x=0\right\}$. Moreover, the space $C_{c}^{\infty}(0, \infty)$ or $C_{c}^{\infty}(0, \infty) \cap\left\{\int_{0}^{\infty} x h(x) \mathrm{d} x=0\right\}$ respectively is a core.

Proof. The statement is a consequence of the Bounded Perturbation Theorem (e.g. [8, Ch. III, Sec. 1.3]) since $\mathcal{B}_{1}=x \partial_{x}$ generates a strongly continuous semigroup according to Lemma 4.6 while $\mathcal{B}_{2}$ and $\mathcal{B}_{3}$ are bounded linear operators as shown in Lemma 6.9. Moreover, the operator $\mathcal{B}$ has been constructed explicitly to preserve the constraint $\int_{0}^{\infty} x f(x) \mathrm{d} x=0$. The statement on the core is a direct consequence of Lemma 4.4 . 
With the preparations above, we can now give the proof of Theorem 6.5.

Proof of Theorem 6.5. As already indicated above, the proof relies on an application of Theorem 5.3, i.e. we have to verify Items $1-3$. We recall from Proposition 6.3 that $\mathcal{L}_{0}$ as given by (4.3) generates a $C_{0}$-semigroup $\mathrm{e}^{\mathcal{L}_{0} t}$ on $L^{2}\left(\mathrm{e}^{\mu x}\right)$ which satisfies for each $\lambda_{1} \leq 1 / 2$ that

$$
\left\|\mathrm{e}^{\mathcal{L}_{0} t} h_{0}\right\|_{L^{2}\left(\mathrm{e}^{\mu x}\right)} \leq C_{\lambda_{1}} \mathrm{e}^{-\lambda_{1} t}\left\|h_{0}\right\|_{L^{2}\left(\mathrm{e}^{\mu x}\right)} \quad \text { for all } h_{0} \in L^{2}\left(\mathrm{e}^{\mu x}\right) \cap\left\{\int_{0}^{\infty} x h_{0}(x) \mathrm{d} x=0\right\}
$$

and all $t \geq 0$. Moreover, $C_{c}^{\infty}(0, \infty)$ is a core for $\mathcal{L}_{0}$ given by (4.3) (see Remark 6.4) and thus, according to Lemma 4.1 the generator $\mathcal{L}_{0}$ is equivalently represented by (4.2), i.e. it generates the same semigroup satisfying (6.3).

It thus remains to verify Item 3 above while we consider first 3a. As already noted, the operator $\mathcal{A}$ has been explicitly constructed such that the first moment is zero. Thus, it only remains to prove the continuity, i.e. that $\mathcal{A}$ is regularising. To see this, we consider $\mathcal{A}_{1}, \mathcal{A}_{2}$ and $\mathcal{A}_{3}$ separately. To begin with $\mathcal{A}_{1}$, we find

$$
\begin{aligned}
& \left\|\mathcal{A}_{1}[h]\right\|_{L^{2}\left(\mathrm{e}^{\mu x}\right)}^{2}=4 \int_{0}^{\infty} \mathrm{e}^{-2 x}\left(\int_{0}^{x} h(y) \chi_{\{y \leq R\}}\left(\mathrm{e}^{y}-1\right) \mathrm{d} y\right)^{2} \mathrm{e}^{\mu x} \mathrm{~d} x \\
& \leq 4\left(\int_{0}^{R}|h(y)|\left(\mathrm{e}^{y}-1\right) \mathrm{d} y\right)^{2} \int_{0}^{\infty} \mathrm{e}^{-(2-\mu) x} \mathrm{~d} x \leq \frac{4 \mathrm{e}^{2 R}}{2-\mu}\left(\int_{0}^{R}|h(y)| \mathrm{d} y\right)^{2} \leq \frac{4 \mathrm{e}^{2 R}}{2-\mu}\|h\|_{L_{k}^{1}}^{2} .
\end{aligned}
$$

For $\mathcal{A}_{2}$ we get similarly

$$
\begin{array}{r}
\left\|\mathcal{A}_{2}[h]\right\|_{L^{2}\left(\mathrm{e}^{\mu x}\right)}^{2}=4 \int_{0}^{\infty} \mathrm{e}^{-2 x}\left(\int_{x}^{\infty} h(y) \mathrm{d} y\right)^{2} \mathrm{e}^{\mu x} \mathrm{~d} x \leq 4\left(\int_{0}^{\infty}|h(y)|^{2} \mathrm{~d} y\right)^{2} \int_{0}^{\infty} \mathrm{e}^{-(2-\mu) x} \mathrm{~d} x \\
=\frac{4}{2-\mu}\left(\int_{0}^{\infty}|h(y)|^{2} \mathrm{~d} y\right)^{2} \leq \frac{4}{2-\mu}\|h\|_{L_{k}^{1}}^{2}
\end{array}
$$

Finally, recalling (6.2) from the proof of Lemma 6.9 we have

$$
\left\|\mathcal{A}_{3}[h]\right\|_{L^{2}\left(\mathrm{e}^{\mu x}\right)}^{2} \leq 4(R+1)^{2(1-k)}\|h\|_{L_{k}^{1}}^{2} \int_{0}^{\infty} \mathrm{e}^{-(2-\mu) x} \mathrm{~d} x=\frac{4}{(2-\mu)(R+1)^{2(k-1)}}\|h\|_{L_{k}^{1}}^{2} .
$$

Summarising (6.4)-(6.6) we obtain

$$
\|\mathcal{A}[h]\|_{L^{2}\left(\mathrm{e}^{\mu x}\right)}^{2} \leq \frac{4}{2-\mu}\left(\mathrm{e}^{2 R}+1+(R+1)^{2(1-k)}\right)\|h\|_{L_{k}^{1}}^{2}
$$

which shows that $\mathcal{A}$ is continuous from $L_{k}^{1}$ to $L^{2}\left(\mathrm{e}^{\mu x}\right)$.

Finally, we prove Item 3 b, i.e. that $\mathcal{B}$ generates a strongly continuous -semigroup $\mathrm{e}^{\mathcal{B} t}$ on $L_{k}^{1}$ which satisfies $\left\|\mathrm{e}^{\mathcal{B} t} h_{0}\right\|_{L_{k}^{1}} \leq C_{3} \mathrm{e}^{-\lambda_{3} t}\left\|h_{0}\right\|_{L_{k}^{1}}$ for all $h_{0} \in L_{k}^{1}$ with $\int_{0}^{\infty} x h_{0}(x) \mathrm{d} x=0$ and $\lambda_{3} \leq 1$. According to Lemma 6.10 the operator $\mathcal{B}$ generates a strongly continuous semigroup on $L_{k}^{1}$ which preserves the constraint on the first moment. Thus, it only remains to prove the indicated estimate on the semigroup and according to Lemma 6.10 we can restrict to the core $C_{c}^{\infty}(0, \infty)$. Thus, for $h_{0} \in C_{c}^{\infty}(0, \infty)$ let $h=h(x, t)=\mathrm{e}^{\mathcal{B} t} h_{0}$ such that $\partial_{t} h=\mathcal{B} h$. We thus have the relation

$$
\partial_{t}\|h\|_{L_{k}^{1}}=\int_{0}^{\infty}\left(\partial_{t} h(x, t)\right) \operatorname{sgn}(h(x, t))(1+x)^{k} \mathrm{~d} x=\int_{0}^{\infty} \mathcal{B}[h](x) \operatorname{sgn}(h(x, t))(1+x)^{k} \mathrm{~d} x .
$$


We require estimates on the right-hand side of this equation. Again, we treat the expressions $\mathcal{B}_{1}, \mathcal{B}_{2}$ and $\mathcal{B}_{3}$ separately and to simplify the notation, we only write $h(x)$, i.e. neglecting the time-dependence, in the following. With $|h|^{\prime}=h^{\prime} \operatorname{sgn}(h)$ integration by parts yields

$$
\begin{aligned}
& \int_{0}^{\infty} \mathcal{B}_{1}[h](x) \operatorname{sgn}(h(x))(1+x)^{k} \mathrm{~d} x=\int_{0}^{\infty}|h(x)|^{\prime} x(1+x)^{k} \mathrm{~d} x \\
& =-\int_{0}^{\infty}|h(x)|\left((1+x)^{k}+k x(1+x)^{k-1}\right) \mathrm{d} x=-\|h\|_{L_{k}^{1}}-k \int_{0}^{\infty}|h(x)| x(1+x)^{k-1} \mathrm{~d} x .
\end{aligned}
$$

Next we consider $\mathcal{B}_{2}$ for which we obtain together with Fubini's theorem and Lemma 6.6 that

$$
\begin{array}{r}
\int_{0}^{\infty} \mathcal{B}_{2}[h](x) \operatorname{sgn}(h(x))(1+x)^{k} \mathrm{~d} x=2 \int_{0}^{\infty} \mathrm{e}^{-x} \int_{0}^{x} h(y) \chi_{\{y>R\}}\left(\mathrm{e}^{y}-1\right) \mathrm{d} y \operatorname{sgn}(h(x))(1+x)^{k} \mathrm{~d} x \\
\leq 2 \int_{R}^{\infty} \mathrm{e}^{-x} \int_{R}^{x}|h(y)|\left(\mathrm{e}^{y}-1\right) \mathrm{d} y(1+x)^{k} \mathrm{~d} x=2 \int_{R}^{\infty}|h(y)|\left(\mathrm{e}^{y}-1\right) \int_{y}^{\infty} \mathrm{e}^{-x}(1+x)^{k} \mathrm{~d} x \mathrm{~d} y \\
\leq 2 \beta \int_{R_{\beta}}^{\infty}|h(y)|\left(1-\mathrm{e}^{-y}\right)(1+y)^{k} \mathrm{~d} y . \quad(6.9)
\end{array}
$$

Finally, recalling (6.2) from the proof of Lemma 6.9 we estimate $\mathcal{B}_{3}$ together with Lemma 6.7 as

$$
\begin{aligned}
& \int_{0}^{\infty} \mathcal{B}_{3}[h](x) \operatorname{sgn}(h(x))(1+x)^{k} \mathrm{~d} x \\
& \leq 2(R+1)^{1-k} \int_{R}^{\infty}|h(y)|(1+y)^{k} \mathrm{~d} y \int_{0}^{\infty} \mathrm{e}^{-x} \operatorname{sgn}(h(x))(1+x)^{k} \mathrm{~d} x \\
& \leq 2(R+1)^{1-k} \int_{R}^{\infty}|h(y)|(1+y)^{k} \mathrm{~d} y \int_{0}^{\infty} \mathrm{e}^{-x}(1+x)^{k} \mathrm{~d} x \\
& \quad \leq \frac{2 \Gamma(k+1)}{\mathrm{e}(R+1)^{k-1}} \int_{R}^{\infty}|h(y)|(1+y)^{k} \mathrm{~d} y .
\end{aligned}
$$

Summarising (6.8)-(6.10) we obtain

$$
\begin{aligned}
& \int_{0}^{\infty} \mathcal{B}[h](x) \operatorname{sgn}(h(x))(1+x)^{k} \mathrm{~d} x \\
& \leq-\|h\|_{L_{k}^{1}}-k \int_{0}^{\infty}|h(x)| x(1+x)^{k-1} \mathrm{~d} x+2 \beta \int_{R_{\beta}}^{\infty}|h(y)|\left(1-\mathrm{e}^{-y}\right)(1+y)^{k} \mathrm{~d} y \\
& \quad+\frac{2 \Gamma(k+1)}{\mathrm{e}\left(R_{\beta}+1\right)^{k-1}} \int_{R_{\beta}}^{\infty}|h(y)|(1+y)^{k} \mathrm{~d} y \\
& =-\|h\|_{L_{k}^{1}}-k \int_{0}^{R_{\beta}}|h(x)| x(1+x)^{k-1} \mathrm{~d} x \\
& \quad+\int_{R_{\beta}}^{\infty}|h(x)|(1+x)^{k-1}\left(2 \beta(1+x)\left(1-\mathrm{e}^{-x}\right)+\frac{2 \Gamma(k+1)(1+x)}{\mathrm{e}\left(R_{\beta}+1\right)^{k-1}}-k x\right) \mathrm{d} x .
\end{aligned}
$$

We fix $\beta>1$ satisfying $k>2 \beta$ (notice this is where the restriction on the values of $k$ comes into play) and choose then $R_{\beta}$ sufficiently large such that

$$
\left(2 \beta(1+x)\left(1-\mathrm{e}^{-x}\right)+\frac{2 \Gamma(k+1)(1+x)}{\mathrm{e}\left(R_{\beta}+1\right)^{k-1}}-k x\right)<0 \quad \text { for all } x \geq R_{\beta} .
$$


The latter is possible if $R_{\beta}$ is large enough to satisfy for example

$$
R_{\beta}>\left(\frac{2 \Gamma(k+1)}{\mathrm{e}(k-2 \beta)}\right)^{\frac{1}{k-1}}-1 \quad \text { and } \quad\left(2 \beta+\frac{\Gamma(k+1)}{\mathrm{e}\left(1+R_{\beta}\right)^{k-1}}-k\right)\left(R_{\beta}+1\right)<-k .
$$

For this choice of $R_{\beta}$, we deduce from (6.11) that

$$
\int_{0}^{\infty} \mathcal{B}[h](x) \operatorname{sgn}(h(x))(1+x)^{k} \mathrm{~d} x \leq-\|h\|_{L_{k}^{1}} .
$$

Recalling (6.7), Grönwall's inequality yields the desired estimate on the semigroup generated by $\mathcal{B}$.

Remark 6.11. The fact that the operator $\mathcal{L}_{0}$ defined by $(4.2)$ as an unbounded operator on $L_{k}^{1}$ is an extension of the same expression defined on $L^{2}\left(\mathrm{e}^{\mu x}\right)$ follows by an argument analogous to that in Remark 6.4. Precisely, from the proof of Proposition 6.3 we know that $C_{c}^{\infty}(0, \infty)$ is a core for $\mathcal{L}_{0}$ on $L^{2}\left(\mathrm{e}^{\mu x}\right)$. Since Lemma 6.9 and the proof of Theorem 5.3 provide that $\mathcal{A}_{2}$ and $\mathcal{B}_{2}$ (for $R=0$ ) are bounded from $L_{k}^{1}$ to itself we deduce from Lemmas 4.4 and 4.6 that $C_{c}^{\infty}(0, \infty)$ is also a core for $\mathcal{L}_{0}$ on $L_{k}^{1}$ and on this common core, both operators coincide.

\section{Uniqueness of profiles}

The spectral gap estimates proved in the previous section allow us to show that small perturbations of the equation for $K=2$ have essentially the same behaviour, at least when solutions are not far from the self-similar profile $e^{-x}$ for $K=2$. We gather all local results of this type in this section.

\subsection{Local stability of profiles}

We call stability of the self-similar profiles with respect to the perturbation we are considering the property that for small $\varepsilon$ the unit-mass profiles are close to the unique unit-mass profile $G_{0}$ for $\varepsilon=0$. We prove now a local version of this result, which states that this is true provided the profiles are contained in a ball of a specific radius around $G_{0}$. Global versions are given in Section 3.4, but the advantage of the local statements we give now is that they give an optimal rate of stability, and they use only the properties of the linearised operator $\mathcal{L}_{0}$.

Our first observation is that the nonlinear operators defining the equation for $\varepsilon=0$ and its perturbation are not far from each other in the $\|\cdot\|_{L_{k}^{1}}$ norm:

Lemma 7.1. Denote by $\mathcal{N}_{\varepsilon}$ the self-similar Smoluchowski operator with kernel $K_{\varepsilon}$; that is,

$$
\mathcal{N}_{\varepsilon}(f):=2 f+x \partial_{x} f+\mathcal{C}_{\varepsilon}(f, f) .
$$

For any $k \geq 0$ and any $f \in L_{k}^{1}$ we have

$$
\left\|\mathcal{N}_{\varepsilon}(f)-\mathcal{N}_{0}(f)\right\|_{L_{k}^{1}} \leq \frac{3}{2} \varepsilon\|f\|_{L_{k}^{1}}^{2} .
$$

Proof. We have

$$
\mathcal{N}_{\varepsilon}(f):=2 f+x \partial_{x} f+\mathcal{C}_{\varepsilon}(f, f)
$$

SO

$$
\left\|\mathcal{N}_{\varepsilon}(f)-N_{0}(f)\right\|_{L_{k}^{1}}=\left\|\mathcal{C}_{\varepsilon}(f, f)-\mathcal{C}_{0}(f, f)\right\|_{L_{k}^{1}}=\varepsilon\left\|\mathcal{C}_{W}(f, f)\right\|_{L_{k}^{1}} \leq \frac{3}{2} \varepsilon\|f\|_{L_{k}^{1}}^{2},
$$

where the last inequality is due to Lemma 2.5. 
We now give our local result on the stability of profiles:

Lemma 7.2 (Local stability of profiles). Take $0 \leq \varepsilon<1$, let $G_{\varepsilon}$ be a self similar profile for the kernel $K_{\varepsilon}$, and assume that $\left\|G_{\varepsilon}-G_{0}\right\| \leq \frac{1}{6 M}$, where $M>0$ is the one in Theorem 6.5. Take $k \geq 0$. There exists an (explicit) constant $M_{1}=M_{1}(k)>0$ such that

$$
\left\|G_{\varepsilon}-G_{0}\right\|_{L_{k}^{1}} \leq \varepsilon M_{1} .
$$

Proof. Denote $\mathcal{N}_{\varepsilon}$ the same operator as in Lemma 7.1, and let $G_{\varepsilon}$ be any self-similar profile for the kernel $K_{\varepsilon}$. Since $\mathcal{N}_{\varepsilon}\left(G_{\varepsilon}\right)=0$ we have

$$
\left\|\mathcal{N}_{0}\left(G_{\varepsilon}\right)\right\|_{L_{k}^{1}}=\left\|\mathcal{N}_{0}\left(G_{\varepsilon}\right)-\mathcal{N}_{\varepsilon}\left(G_{\varepsilon}\right)\right\|_{L_{k}^{1}} \leq \frac{3}{2} \varepsilon\left\|G_{\varepsilon}\right\|_{L_{k}^{1}}^{2},
$$

where we have used Lemma 7.1. Now

$$
\mathcal{N}_{0}\left(G_{\varepsilon}\right)=\mathcal{L}_{0}\left(G_{\varepsilon}-G_{0}\right)+\mathcal{C}_{0}\left(G_{\varepsilon}-G_{0}, G_{\varepsilon}-G_{0}\right)
$$

so

$$
\begin{aligned}
& \left\|\mathcal{N}_{0}\left(G_{\varepsilon}\right)\right\|_{L_{k}^{1}}=\left\|\mathcal{L}_{0}\left(G_{\varepsilon}-G_{0}\right)+\mathcal{C}_{0}\left(G_{\varepsilon}-G_{0}, G_{\varepsilon}-G_{0}\right)\right\|_{L_{k}^{1}} \\
& \quad \geq\left\|\mathcal{L}_{0}\left(G_{\varepsilon}-G_{0}\right)\right\|_{L_{k}^{1}}-\left\|\mathcal{C}_{0}\left(G_{\varepsilon}-G_{0}, G_{\varepsilon}-G_{0}\right)\right\|_{L_{k}^{1}} \\
& \quad \geq \frac{1}{M}\left\|G_{\varepsilon}-G_{0}\right\|_{L_{k}^{1}}-3\left\|G_{\varepsilon}-G_{0}\right\|_{L_{k}^{1}}^{2} .
\end{aligned}
$$

Together with (7.1) this gives

$$
\frac{1}{M}\left\|G_{\varepsilon}-G_{0}\right\|_{L_{k}^{1}} \leq \frac{3}{2} \varepsilon\left\|G_{\varepsilon}\right\|_{L_{k}^{1}}^{2}+3\left\|G_{\varepsilon}-G_{0}\right\|_{L_{k}^{1}}^{2} .
$$

If we assume that $\left\|G_{\varepsilon}-G_{0}\right\|_{L_{k}^{1}} \leq \frac{1}{6 M}$ then this implies

$$
\frac{1}{2 M}\left\|G_{\varepsilon}-G_{0}\right\|_{L_{k}^{1}} \leq \frac{3}{2} \varepsilon\left\|G_{\varepsilon}\right\|_{L_{k}^{1}}^{2}
$$

Proposition 3.3 then shows that the right hand side is finite and depends only on $k$.

As an immediate consequence of Lemma 7.2 and Proposition 3.9, we then also obtain the following global stability result (notice that in Section 3.4 the constant in Proposition 3.9 was explicitly estimated, so the $D_{k}$ in the following result is constructive). Also, we remark that a global result like Proposition 3.9 is essential here, since the stability of all possible solutions to the self-similar equation cannot be proved by studying only its linearisation.

Corollary 7.3 (Global stability of profiles). For each $k \geq 0$ there exists a constant $D_{k}$ such that each self-similar profile $G_{\varepsilon}$ for the kernel $K_{\varepsilon}$ with $0 \leq \varepsilon \leq 1$ satisfies

$$
\left\|G_{\varepsilon}-G_{0}\right\|_{L_{k}^{1}} \leq D_{k} \varepsilon .
$$




\subsection{Uniqueness}

We first show a local uniqueness result which states that self-similar profiles are unique for small epsilon, provided they are within a certain distace of $G_{0}$. As in the previous results in this section, we prefer to state this local result because it depends only on linearisation arguments involving the operator $\mathcal{L}_{0}$. Using the stability results in Section 3.4, it immediately gives a full uniqueness result.

In order to state our local uniqueness result we first show that the perturbed linear operators $\mathcal{L}_{\varepsilon}$ around a self-similar profile $G_{\varepsilon}$ which is in a certain ball around $G_{0}$, also have a spectral gap in the $L_{k}^{1}$ spaces for small $\varepsilon$. This is not strictly needed, but it makes the later proof a bit easier. Notice that the operators $\mathcal{L}_{\varepsilon}$ are just bounded perturbations of the operator $\mathcal{L}_{0}$, so it is understood that they are defined in the same way as in Theorem 4.2, with the same domain.

Lemma 7.4. Take $k \geq 0$ and $0<\varepsilon<1$, and call $\mathcal{L}_{\varepsilon}$ the linearised self-similar Smoluchowski operator in the space $L_{k}^{1}$, with kernel $K_{\varepsilon}$, around a given self-similar profile $G_{\varepsilon}$ with mass 1 . There is an explicit constant $M_{2}=M_{2}(k)$ such that

$$
\left\|\mathcal{L}_{\varepsilon}-\mathcal{L}_{0}\right\| \leq \varepsilon M_{2}
$$

Proof. In terms of $\mathcal{C}_{K}$ as given in (2.3) the operators $\mathcal{L}_{\varepsilon}$ and $\mathcal{L}_{0}$ read

$$
\begin{aligned}
& \mathcal{L}_{0}[h](x)=2 h+x h^{\prime}+2 \mathcal{C}_{0}\left(G_{0}, h\right) \\
& \mathcal{L}_{\varepsilon}[h](x)=2 h+x h^{\prime}+2 \mathcal{C}_{\varepsilon}\left(G_{\varepsilon}, h\right) .
\end{aligned}
$$

This yields

$$
\mathcal{L}_{\varepsilon}[h](x)-\mathcal{L}_{0}[h](x)=2 \mathcal{C}_{\varepsilon}\left(G_{\varepsilon}, h\right)-2 \mathcal{C}_{0}\left(G_{0}, h\right)=2 \mathcal{C}_{0}\left(G_{\varepsilon}-G_{0}, h\right)+2 \varepsilon \mathcal{C}_{W}\left(G_{\varepsilon}, h\right) .
$$

With Propositions 2.5 and 3.3 and Corollary 7.3 we thus deduce

$$
\left\|\mathcal{L}_{\varepsilon}[h]-\mathcal{L}_{0}[h]\right\|_{L_{k}^{1}} \leq 6\left\|G_{\varepsilon}-G_{0}\right\|_{L_{k}^{1}}\|h\|_{L_{k}^{1}}+3 \varepsilon\left\|G_{\varepsilon}\right\|_{L_{k}^{1}}\|h\|_{L_{k}^{1}} \leq\left(6 D_{k} \varepsilon+3 C_{k} \varepsilon\right)\|h\|_{L_{k}^{1}} .
$$

According to Proposition 3.3 and Corollary 7.3 the coefficient $M_{2}(k):=\left(6 D_{k}+3 C_{k}\right)$ depends only on $k$, which proves the result.

Lemma 7.5 (Spectral gap of $\mathcal{L}_{\varepsilon}$ ). Let $\mathcal{L}_{\varepsilon}$ be the linearised self-similar Smoluchowski operator with kernel $K_{\varepsilon}$, around a given self-similar profile $G_{\varepsilon}$ with mass 1 and $k>2$. Then for $\varepsilon<\frac{1}{2 C M_{2}}=: \varepsilon_{0}$, where $M_{2}=M_{2}(k)$ is from Lemma 7.4 and $C=C(k)$ is from Theorem 6.5, the operator $\mathcal{L}_{\varepsilon}$ has a spectral gap in $L_{k}^{1}$ of size $1 / 2-C M_{2} \varepsilon$. That is: with the same $C=C(k)$ from Theorem 6.5 we have

$$
\left\|e^{t \mathcal{L}_{\varepsilon}} h_{0}\right\|_{L_{k}^{1}} \leq C\left\|h_{0}\right\|_{L_{k}^{1}} e^{-\left(\frac{1}{2}-C M_{2} \varepsilon\right) t}, \quad t \geq 0
$$

for all $h_{0} \in L_{k}^{1}$ with $\int x h_{0}=0$.

We sometimes state the result above by saying that, under these conditions, the linearised operator $\mathcal{L}_{\varepsilon}$ has a spectral gap in $L_{k}^{1}$ of size $1 / 2-C M_{2} \varepsilon$.

Remark 7.6. In particular, under the assumptions of the previous result, by the Hille-Yosida theorem we have

$$
\left\|\mathcal{L}_{\varepsilon} h\right\|_{L_{k}^{1}} \geq \frac{1-2 C M_{2} \varepsilon}{2 C}\|h\|_{L_{k}^{1}}
$$

for all $h \in L_{k}^{1}$ with $\int x h(x) \mathrm{d} x=0$. 
Proof of Lemma 7.5. All norms used in this proof are $\|\cdot\|_{L_{k}^{1}}$, and we omit the subscript to simplify the notation. From Lemma 7.4 we have

$$
\left\|\mathcal{L}_{\varepsilon}-\mathcal{L}_{0}\right\| \leq \varepsilon M_{2}=: \delta
$$

We consider the equation

$$
\partial_{t} h=\mathcal{L}_{\varepsilon} h=\mathcal{L}_{0} h+\left(\mathcal{L}_{\varepsilon}-\mathcal{L}_{0}\right) h
$$

and write, using Duhamel's formula and setting $h_{t}:=e^{t \mathcal{L}_{\varepsilon}} h_{0}$,

$$
h_{t}=e^{t \mathcal{L}_{0}} h_{0}+\int_{0}^{t} e^{(t-s) \mathcal{L}_{0}}\left(\mathcal{L}_{\varepsilon}-\mathcal{L}_{0}\right) h_{s} \mathrm{~d} s .
$$

Hence, using Theorem 6.5 for $\mathcal{L}_{0}$

$$
\left\|h_{t}\right\| \leq C e^{-\frac{1}{2} t}\left\|h_{0}\right\|+C \int_{0}^{t} e^{-\frac{1}{2}(t-s)}\left\|\left(\mathcal{L}_{\varepsilon}-\mathcal{L}_{0}\right) h_{s}\right\| \mathrm{d} s .
$$

Hence, from (7.3),

$$
\left\|h_{t}\right\| \leq C e^{-\frac{1}{2} t}\left\|h_{0}\right\|+C \delta \int_{0}^{t} e^{-\frac{1}{2}(t-s)}\left\|h_{s}\right\| \mathrm{d} s .
$$

Calling $u(t):=\left\|h_{t}\right\| e^{\frac{1}{2} t}$ we see that

$$
u(t) \leq C\left\|h_{0}\right\|+C \delta \int_{0}^{t} u(s) \mathrm{d} s,
$$

so by Gronwall's Lemma we have $u(t) \leq C\left\|h_{0}\right\| e^{C \delta t}$, that is

$$
\left\|h_{t}\right\| \leq C\left\|h_{0}\right\| e^{\left(C \delta-\frac{1}{2}\right) t}, \quad t \geq 0 .
$$

Recalling (7.3) this shows the claim.

We can finally give the proof of local uniqueness of the profiles:

Theorem 7.7 (Local uniqueness of self-similar profiles). Take any $k>2$. For all $0<\varepsilon<\varepsilon_{0}$ (with $\varepsilon_{0}$ from Lemma 7.5), Smoluchowski's coagulation equation with kernel $K_{\varepsilon}$ has at most one self-similar profile $G_{\varepsilon}$ with mass 1 satisfying

$$
\left\|G_{\varepsilon}-G_{0}\right\|_{L_{k}^{1}} \leq \frac{1-\varepsilon / \varepsilon_{0}}{12 C(1+\varepsilon)}
$$

with $C=C(k)$ from Theorem 6.5.

Proof. Let $\mathcal{N}_{\varepsilon}$ be the the self-similar Smoluchowski operator with kernel $K_{\varepsilon}$. Assume we have two different self-similar profiles $G_{1}, G_{2}$ with mass 1 for the kernel $K_{\varepsilon}$ :

$$
\mathcal{N}_{\varepsilon}\left(G_{1}\right)=\mathcal{N}_{\varepsilon}\left(G_{2}\right)=0,
$$

and that they both satisfy

$$
\left\|G_{1}-G_{0}\right\|_{L_{k}^{1}} \leq \frac{1-\varepsilon / \varepsilon_{0}}{12 C(1+\varepsilon)}, \quad\left\|G_{2}-G_{0}\right\|_{L_{k}^{1}} \leq \frac{1-\varepsilon / \varepsilon_{0}}{12 C(1+\varepsilon)} .
$$


Call $\mathcal{L}_{\varepsilon}$ the linearised self-similar Smoluchowski operator with kernel $K_{\varepsilon}$, around the profile $G_{1}$. Since $\mathcal{N}_{\varepsilon}(f)=\mathcal{L}_{\varepsilon}\left(f-G_{1}\right)+\mathcal{C}_{2+\varepsilon W}\left(f-G_{1}, f-G_{1}\right)$,

$$
0=\mathcal{N}_{\varepsilon}\left(G_{1}\right)-\mathcal{N}_{\varepsilon}\left(G_{2}\right)=\mathcal{L}_{\varepsilon}\left(G_{2}-G_{1}\right)-\mathcal{C}_{2+\varepsilon W}\left(G_{1}-G_{2}, G_{1}-G_{2}\right) .
$$

Using Lemma 7.5 (see equation (7.2) in particular) and Proposition 2.5,

$$
\frac{1-\varepsilon / \varepsilon_{0}}{2 C}\left\|G_{1}-G_{2}\right\| \leq\left\|\mathcal{L}_{\varepsilon}\left(G_{2}-G_{1}\right)\right\| \leq \frac{3}{2}(1+\varepsilon)\left\|G_{1}-G_{2}\right\|^{2},
$$

so, since $G_{1} \neq G_{2}$, and assuming always $\varepsilon<1$,

$$
\left\|G_{1}-G_{2}\right\| \geq \frac{1-\varepsilon / \varepsilon_{0}}{3 C(1+\varepsilon)}
$$

This contradicts (7.4), since

$$
\left\|G_{1}-G_{2}\right\| \leq\left\|G_{1}-G_{0}\right\|+\left\|G_{0}-G_{2}\right\| \leq \frac{1-\varepsilon / \varepsilon_{0}}{6 C(1+\varepsilon)} .
$$

We can then use this local result for any fixed $k>2$, together with the stability results in Section 3.4, to obtain that there is a unique unit-mass self-similar profile in $L_{k}^{1}$. Since we know from Section 3.1 that all profiles must be in $L_{k}^{1}$, we immediately obtain a uniqueness result:

Corollary 7.8 (Uniqueness of profiles for small perturbations). There exists $\varepsilon_{1}>0$ such that for all $0 \leq \varepsilon \leq \varepsilon_{1}$ Smoluchowski's coagulation equation with kernel $K_{\varepsilon}$ has exactly one self-similar profile $G_{\varepsilon}$ with mass 1 .

\section{Convergence to equilibrium}

\subsection{Local convergence to equilibrium}

Proposition 8.1 (Local exponential convergence to equilibrium). Take $k>2$, and consider $\varepsilon_{1}$ from Corollary 7.8. For any $0 \leq \varepsilon<\varepsilon_{1}$, let $G_{\varepsilon}$ be the unique self-similar profile with mass 1 for the kernel $K_{\varepsilon}$. There exist constants $C_{*}, M, \varepsilon_{2}, r_{1}>0$ depending on $k$ only such that for any $0 \leq \varepsilon \leq \varepsilon_{2}$, any solution $f$ to the self-similar Smoluchowski equation (2.1) with kernel $K_{\varepsilon}$ with initial condition $f_{0} \in L_{k}^{1}$ such that

$$
\int_{0}^{\infty} x f_{0}(x) \mathrm{d} x=1, \quad\left\|f_{0}-G_{\varepsilon}\right\|_{L_{k}^{1}} \leq r_{1}
$$

satisfies

$$
\left\|f(t, \cdot)-G_{\varepsilon}\right\|_{L_{k}^{1}} \leq C_{*} e^{-\left(\frac{1}{2}-M \varepsilon\right) t}\left\|f_{0}-G_{\varepsilon}\right\|_{L_{k}^{1}} \quad \text { for all } t \geq 0 .
$$

Proof. Since we have information on the spectral properties of the linearised operator $\mathcal{L}_{\varepsilon}$ around the profile $G_{\varepsilon}$, the proof becomes a standard perturbation argument: we write the self-similar Smoluchowski equation as

$$
\partial_{t} f=\mathcal{C}_{2+\varepsilon W}(f, f)+2 f+x \partial_{x} f=\mathcal{L}_{\varepsilon}\left(f-G_{\varepsilon}\right)+\mathcal{C}_{2+\varepsilon W}\left(f-G_{\varepsilon}, f-G_{\varepsilon}\right) .
$$

By Duhamel's formula, calling $h:=f-G_{\varepsilon}$,

$$
h_{t}=e^{t \mathcal{L}_{\varepsilon}} h_{0}+\int_{0}^{t} e^{(t-s) \mathcal{L}_{\varepsilon}}\left(\mathcal{C}_{2+\varepsilon W}\left(h_{s}, h_{s}\right)\right) \mathrm{d} s,
$$


so, using Lemma 7.5 and Proposition 2.5 and denoting $\lambda_{\varepsilon}:=1 / 2-C M_{2} \varepsilon$ (with $C$ and $M_{2}$ from Lemma 7.5),

$$
\begin{aligned}
&\left\|h_{t}\right\|_{L_{k}^{1}} \leq C e^{-\lambda_{\varepsilon} t}\left\|h_{0}\right\|_{L_{k}^{1}}+C \int_{0}^{t} e^{-\lambda_{\varepsilon}(t-s)}\left\|\mathcal{C}_{2+\varepsilon W}\left(h_{s}, h_{s}\right)\right\|_{L_{k}^{1}} \mathrm{~d} s \\
& \leq C e^{-\lambda_{\varepsilon} t}\left\|h_{0}\right\|_{L_{k}^{1}}+2 C\left\|K_{\varepsilon}\right\|_{\infty} \int_{0}^{t} e^{-\lambda_{\varepsilon}(t-s)}\left\|h_{s}\right\|_{L_{k}^{1}}^{2} \mathrm{~d} s .
\end{aligned}
$$

If we define $u(t):=\|h(t, \cdot)\|_{L_{k}^{1}} e^{\lambda_{\varepsilon} t}$ we have

$$
u(t) \leq C u(0)+2 C\left\|K_{\varepsilon}\right\|_{\infty} \int_{0}^{t} u(s)^{2} e^{-\lambda_{\varepsilon} s} \mathrm{~d} s .
$$

Gronwall's lemma applied to this integral inequality then shows that

$$
u(t) \leq\left(\frac{1}{C u(0)}-\frac{2 C\left\|K_{\varepsilon}\right\|_{\infty}}{\lambda_{\varepsilon}}\left(1-e^{-\lambda_{\varepsilon} t}\right)\right)^{-1},
$$

which remains bounded for all $t \geq 0$ if

$$
u(0)<\frac{\lambda_{\varepsilon}}{2 C^{2}\left\|K_{\varepsilon}\right\|_{\infty}} .
$$

For example, if we assume

$$
u(0)<\frac{\lambda_{\varepsilon}}{4 C^{2}\left\|K_{\varepsilon}\right\|_{\infty}}
$$

then

$$
u(t) \leq 2 C u(0) \quad \text { for all } t \geq 0,
$$

which implies

$$
\|h(t, \cdot)\| \leq 2 C e^{-\lambda_{\varepsilon} t}\left\|h_{0}\right\|,
$$

which is what we wanted to show.

\subsection{Convergence to equilibrium in large regions}

If we additionally use our knowledge that solutions to the unperturbed problem with kernel $K=2$ converge to equilibrium globally we can obtain a slight improvement of the above result. Namely, that the size $R$ of the region in which we have convergence can be taken as large as one wants, provided $\varepsilon$ is close enough to zero:

Theorem 8.2 (Exponential convergence to equilibrium in large regions for small $\varepsilon$ ). Let $k>2$ and $W$ be a bounded kernel of homogeneity 0 , take $R>0$, and take $0 \leq \varepsilon \leq \varepsilon_{1}$ (with $\varepsilon_{1}$ the one from Corollary 7.8 ensuring uniqueness of profiles). Denote $K_{\varepsilon}:=2+\varepsilon W$, and call $G_{\varepsilon}$ the unique self-similar profile with mass 1 for the kernel $K_{\varepsilon}$. There exist constants $C$ and $M$ (depending only on $k$ ) and $\varepsilon_{3}>0$ (depending on $W, R$ and $k$ ) such that any solution $f$ to the self-similar Smoluchowski equation (2.1) with kernel $K_{\varepsilon}$ with $0 \leq \varepsilon \leq \varepsilon_{3}$ and initial condition $f_{0} \in L_{k}^{1}$ with $f_{0} \geq 0$ almost everywhere and

$$
\int_{0}^{\infty} x f_{0}(x) \mathrm{d} x=1, \quad\left\|f_{0}-G_{\varepsilon}\right\| \leq R
$$

satisfies

$$
\left\|f(t, \cdot)-G_{\varepsilon}\right\| \leq C e^{-\left(\frac{1}{2}-M \varepsilon\right) t}\left\|f_{0}-G_{\varepsilon}\right\| \quad \text { for all } t \geq 0 .
$$


Proof. The idea that we want to exploit is that for small $\varepsilon$, solutions to our perturbed equation are not too far from solutions to the equation for the constant kernel. Since we know that the equation for the constant kernel converges to equilibrium exponentially fast, we can show that solutions to the perturbed equation will eventually fall inside the local region where we can apply Proposition 8.1.

For $R>0$ given, and any $\varepsilon>0$, take $k>2$ and any nonnegative initial condition $f_{0} \in L_{k}^{1}$ with mass 1 and $\left\|f_{0}-G_{\varepsilon}\right\| \leq R$. Call $f^{\varepsilon}$ the solution to the self-similar Smoluchowski equation with kernel $K_{\varepsilon}$, and $f$ the solution to the self-similar Smoluchowski equation with constant kernel $K_{0}=2$, both with initial condition $f_{0}$. From Lemma 2.15 we know that these two solutions remain close for some time: for some $C_{3}, C_{4}>0$,

$$
\left\|f^{\varepsilon}(t, \cdot)-f(t, \cdot)\right\|_{L_{k}^{1}} \leq C_{3} \varepsilon e^{C_{4} t} .
$$

Also, according to Theorem 2.16 the solution $f$ converges exponentially fast to $G_{0}(x)=e^{-x}$ :

$$
\left\|f(t, \cdot)-G_{0}\right\|_{L_{k}^{1}} \leq C_{5} e^{-\frac{1}{2} t} .
$$

Hence together with Corollary 7.3

$$
\begin{aligned}
\left\|f^{\varepsilon}(t, \cdot)-G_{\varepsilon}\right\|_{L_{k}^{1}} \leq\left\|f^{\varepsilon}(t, \cdot)-f(t, \cdot)\right\|_{L_{k}^{1}}+\left\|f(t, \cdot)-G_{0}\right\|_{L_{k}^{1}}+ & \left\|G_{0}-G_{\varepsilon}\right\|_{L_{k}^{1}} \\
& \leq C_{3} \varepsilon e^{C_{4} t}+C_{5} e^{-\frac{1}{2} t}+D_{k} \varepsilon .
\end{aligned}
$$

We can choose large enough $t$ (which we call $t_{0}$ ), and then small enough $\varepsilon$, so that this quantity is less than the $r_{1}$ in Proposition 8.1. Then, from Proposition 8.1,

$$
\left\|f^{\varepsilon}(t, \cdot)-G_{\varepsilon}\right\|_{L_{k}^{1}} \leq C e^{-(1 / 2-M \varepsilon)\left(t-t_{0}\right)}\left\|f^{\varepsilon}\left(t_{0}, \cdot\right)-G_{\varepsilon}\right\|_{L_{k}^{1}} \quad \text { for all } t \geq t_{0} .
$$

It is also easy to see that, for some $C_{6}>0$,

$$
\left\|f^{\varepsilon}\left(t_{0}, \cdot\right)-G_{\varepsilon}\right\|_{L_{k}^{1}} \leq e^{C_{6} t_{0}}\left\|f_{0}-G_{\varepsilon}\right\|_{L_{k}^{1}},
$$

which then gives

$$
\left\|f^{\varepsilon}(t, \cdot)-G_{\varepsilon}\right\|_{L_{k}^{1}} \leq C e^{-(1 / 2-M \varepsilon)\left(t-t_{0}\right)} e^{C_{6} t_{0}}\left\|f_{0}-G_{\varepsilon}\right\|_{L_{k}^{1}} \quad \text { for all } t \geq t_{0} .
$$

This shows the result for $t \geq t_{0}$, and for $t \leq t_{0}$ we can easily obtain

$$
\left\|f^{\varepsilon}(t, \cdot)-G_{\varepsilon}\right\|_{L_{k}^{1}} \leq e^{C_{7} t}\left\|f_{0}-G_{\varepsilon}\right\|_{L_{k}^{1}}
$$

by similar calculations as in Proposition 8.1, using that we already know from 2.11 that $\left\|f^{\varepsilon}(t, \cdot)\right\|_{L_{k}^{1}}$ is uniformly bounded for all times. This is enough to obtain the result.

\section{Acknowledgements}

JAC and ST were supported by project MTM2017-85067-P, funded by the Spanish government and the European Regional Development Fund. ST has been funded by the Deutsche Forschungsgemeinschaft (DFG, German Research Foundation) - Projektnummer 396845724. The authors would like to acknowledge the support of the Hausdorff Institute for Mathematics, since part of this work was completed during their stay at the Trimester Program on kinetic theory. 


\section{A Proof of Theorem 2.16 on $L^{2}$ convergence for the constant kernel}

We gather here the proof of Theorem 2.16, which is a small modification of [7, Lemma 6.1]. Since the proof is independent of the rest of the paper and is a small improvement of the aforementioned one, we prefer to give it in an appendix.

Our starting point is [7, Lemma 6.1]:

Lemma A.1. Let $f$ be a solution to (2.1) for the constant kernel $K=2$ with total mass 1 and initial condition $f_{0}$ such that $f_{0} \in L^{2}(\mathrm{~d} x) \cap L^{1}\left(x^{2} \mathrm{~d} x\right)$. Let $G^{0}(x)=\mathrm{e}^{-x}$ be the unique stationary solution to (2.1) with total mass 1 . There exist constants $C, T>0$ depending only on $f_{0}$ such that

$$
\left\|f(t, \cdot)-G^{0}\right\|_{L^{2}} \leq C \mathrm{e}^{-\frac{1}{2} t} \quad \text { for all } t \geq T .
$$

We want to make two modifications to this statement, namely: 1. that a bound can be given for all $t \geq 0$, and 2 . that the constants can be explicitly calculated and depend only on $\left\|f_{0}\right\|_{L^{2}}$ and $\left\|f_{0}\right\|_{L_{2}^{1}}$. The first modification is very simple, and we give it first:

Lemma A.2. In the conditions of Lemma A.1, there exists a constant $C>0$ depending only on $f_{0}$ such that

$$
\left\|f(t, \cdot)-G^{0}\right\|_{L^{2}} \leq C \mathrm{e}^{-\frac{1}{2} t} \quad \text { for all } t \geq 0 .
$$

Proof. For $t \geq T$ it is clearly true from Lemma A.1. For $0 \leq t \leq T$ we can use any available bound on the growth of the $L^{2}$ norm of a solution. For example, one can easily calculate that

$$
\begin{aligned}
\frac{1}{2} \frac{\mathrm{d}}{\mathrm{dt}}\|f\|_{L^{2}}^{2}=\frac{3}{2}\|f\|_{L^{2}}^{2}+\int_{0}^{\infty} \int_{0}^{x} f(x) f(x-y) f(y) \mathrm{d} y \mathrm{~d} x & -\|f\|_{L^{2}}^{2} \int_{0}^{\infty} f(x) \mathrm{d} x \\
& \leq \frac{3}{2}\|f\|_{L^{2}}^{2}+\|f\|_{L^{2}}^{2} \int_{0}^{\infty} f(x) \mathrm{d} x,
\end{aligned}
$$

where we have used Cauchy-Schwarz's inequality on the integral term and disregarded the negative one. Since $\int f$ can be calculated explicitly, we see $\int f \leq \max \left\{1, \int f_{0}\right\}=: C_{1}$, so

$$
\|f\|_{L^{2}}^{2} \leq\left\|f_{0}\right\|_{L^{2}}^{2} \exp \left(\left(3+2 C_{1}\right) t\right) \quad \text { for all } t \geq 0 .
$$

In particular, for all $0 \leq t \leq T$,

$$
\|f\|_{L^{2}} \leq\left\|f_{0}\right\|_{L^{2}} \exp \left(\left(\frac{3}{2}+C_{1}\right) T\right) \mathrm{e}^{\frac{T}{2}} \mathrm{e}^{-\frac{t}{2}}=\left\|f_{0}\right\|_{L^{2}} \exp \left(\left(2+C_{1}\right) T\right) \mathrm{e}^{-\frac{t}{2}},
$$

so

$$
\left\|f-G^{0}\right\|_{L^{2}} \leq\|f\|_{L^{2}}+\left\|G^{0}\right\|_{L^{2}} \leq\left\|f_{0}\right\|_{L^{2}} \exp \left(\left(2+C_{1}\right) T\right) \mathrm{e}^{-\frac{t}{2}}+\frac{1}{2} .
$$

We obtain then

$$
\left\|f(t, \cdot)-G^{0}\right\|_{L^{2}} \leq C_{2} \mathrm{e}^{-\frac{1}{2} t} \quad \text { for all } t \geq 0,
$$

with

$$
C_{2}:=\max \left\{C, \frac{1}{2}+\left\|f_{0}\right\|_{L^{2}}^{2} \exp \left(\left(2+C_{1}\right) T\right)\right\},
$$

where $C$ and $T$ are those from Lemma A.1. 
The final version we want to give is Theorem 2.16, which is the same as Lemma A.2 with the addition that the constant $C$ depends only on $\left\|f_{0}\right\|_{L^{2}}$ and $\left\|f_{0}\right\|_{L_{2}^{1}}$. Let us give the proof of this:

Proof of Theorem 2.16. We notice that for $t \in[0, T]$, the constant we obtain in the proof of Lemma A.2 depends only on $\left\|f_{0}\right\|_{L^{2}}$ and $\int_{0}^{\infty} f_{0}$ (increasingly), so it can be made to depend only on $\left\|f_{0}\right\|_{L^{2}}$ and $\left\|f_{0}\right\|_{L_{2}^{1}}$, as we want. Hence we just need to check that the constants obtained in the proof of [7, Lemma 6.1] depend only on the specified norms of $f_{0}$. One may assume that $\int f_{0}=1$, since one can always reduce the proof to that case by a change of variables. One can see from the proof in [7] that all constants are explicit, except for the one called $\varepsilon_{2}$, defined by

$$
\varepsilon_{2}:=\inf _{|\xi|>\varepsilon_{1}}\left|1-\phi_{0}(\xi)\right|,
$$

where $\varepsilon_{1}$ is a quantity that depends only on $\left\|f_{0}\right\|_{L_{2}^{1}}$, and $\phi_{0}$ is the Fourier transform of $f_{0}$ :

$$
\phi_{0}(\xi):=\int_{0}^{\infty} e^{-i \xi x} f_{0}(x) \mathrm{d} x, \quad \xi \in \mathbb{R} .
$$

(Notice that we have adapted the definition of $\varepsilon_{2}$ to our current choice of constant kernel $K=2$ instead of $K=1$, as used in [7]; this is not essential). We need then to find an explicit lower bound of $\varepsilon_{2}$ that depends only on $\varepsilon_{1},\left\|f_{0}\right\|_{L^{2}}$ and $\left\|f_{0}\right\|_{L_{2}^{1}}$. This is given by Lemma B.4, which we prove in the remaining part of this appendix.

\section{B An estimate on the Fourier transform}

In order to estimate the $\varepsilon_{2}$ in (A.1) we need to understand the following. The Fourier transform of $f_{0}$ is always less than or equal to 1 in absolute value, since $f_{0} \geq 0$ with integral 1 . Its absolute value is never equal to 1 except for at the mode $\xi=0$, and we need to find a quantitative estimate of this phenomenon. Our final result is given in Lemma B.4, but we will need a few lemmas to arrive there. The next one contains the central part of the argument:

Lemma B.1. Given $R>0$, take a nonnegative function $f \in L^{1}(-R, R) \cap L^{2}(-R, R)$. Then

$$
\int_{-R}^{R} f(x) \sin (x) \mathrm{d} x \leq(1-\alpha) \int_{-R}^{R} f(x) \mathrm{d} x
$$

where

$$
\alpha:=\frac{M^{4}}{128 \pi n^{2}\|f\|_{L^{2}}^{4}}, \quad n:=1+\frac{R}{2 \pi}, \quad M:=\int_{-R}^{R} f(x) \mathrm{d} x .
$$

Proof. It is clearly enough to prove it in the case $\int_{-R}^{R} f(x) \mathrm{d} x=1$, so we make this assumption throughout. For $0<\varepsilon<\frac{\pi}{2}$ to be fixed later, we call $A_{\varepsilon}$ the $\varepsilon$-neighbourhood of the points in $[-R, R]$ where $\sin x=1$ :

$$
A_{\varepsilon}:=\left\{x \in[-R, R]|| x-\frac{(4 k+1) \pi}{2} \mid<\varepsilon \text { for some odd integer } k\right\},
$$

and $B_{\varepsilon}$ its complement in $[-R, R]$ :

$$
B_{\varepsilon}:=[-R, R] \backslash A_{\varepsilon} .
$$


Since $B_{\varepsilon}$ does not contain the points where $\sin x=1$, there is a positive function $\delta=\delta(\varepsilon)$ such that

$$
\int_{B_{\varepsilon}} f(x) \sin x \mathrm{~d} x \leq(1-\delta(\varepsilon)) \int_{B_{\varepsilon}} f(x) \mathrm{d} x .
$$

For example, Lemma B.2 gives a simple explicit bound of $\delta(\varepsilon)$. For convenience, we call

$$
m_{A}:=\int_{A_{\varepsilon}} f(x) \mathrm{d} x, \quad m_{A}:=\int_{B_{\varepsilon}} f(x) \mathrm{d} x=1-m_{A} .
$$

Hence,

$$
\begin{aligned}
\int_{-R}^{R} f(x) \sin x \mathrm{~d} x & =\int_{A_{\varepsilon}} f(x) \sin x \mathrm{~d} x+\int_{B_{\varepsilon}} f(x) \sin x \mathrm{~d} x \\
& \leq m_{A}+(1-\delta(\varepsilon)) m_{B}=m_{A}+(1-\delta(\varepsilon))\left(1-m_{A}\right)=1-\delta(\varepsilon)\left(1-m_{A}\right) .
\end{aligned}
$$

Now, by Cauchy-Schwarz's inequality we notice that

$$
m_{A}=\int_{A_{\varepsilon}} f(x) \mathrm{d} x \leq\|f\|_{L^{2}} \sqrt{\left|A_{\varepsilon}\right|} \leq\|f\|_{L^{2}} \sqrt{2\left(1+\frac{R}{2 \pi}\right) \varepsilon}
$$

since the Lebesgue measure of $A_{\varepsilon}$ is at most $2\left(1+\frac{R}{2 \pi}\right) \varepsilon$. For convenience, call $n:=1+\frac{R}{2 \pi}$. We then choose $\varepsilon$ such that

$$
\|f\|_{L^{2}} \sqrt{2 n \varepsilon}=\frac{1}{2}, \quad \text { that is, } \quad \varepsilon:=\left(8 n\|f\|_{L^{2}}^{2}\right)^{-1}
$$

and we obtain

$$
\int_{-R}^{R} f(x) \sin x \mathrm{~d} x \leq 1-\frac{1}{2} \delta(\varepsilon) .
$$

Using our bound of $\delta(\varepsilon)$ from Lemma B.2 we finally obtain the result.

Lemma B.2. For every $0<\varepsilon<\pi / 2$,

$$
\delta(\varepsilon):=1-\sup _{0<x<\frac{\pi}{2}-\varepsilon}(\sin x) \geq \frac{\varepsilon^{2}}{\pi} .
$$

Proof. It is easy to check that

$$
\sin x \leq 1-\frac{\left(x-\frac{\pi}{2}\right)^{2}}{\pi} \quad \text { for all } 0 \leq x \leq \frac{\pi}{2},
$$

which easily implies the statement.

Lemma B.3. Take a nonnegative function $f \in L^{1}(\mathbb{R}) \cap L^{2}(\mathbb{R})$ with $\int_{-\infty}^{\infty}|x| f(x) \mathrm{d} x<+\infty$. Then

$$
\int_{-\infty}^{+\infty} f(x) \sin (x) \mathrm{d} x \leq(1-\alpha) \int_{-\infty}^{+\infty} f(x) \mathrm{d} x
$$

where

$$
\alpha:=\frac{M^{6}}{2^{17} R^{2}\|f\|_{L^{2}}^{4}}, \quad M:=\int_{-\infty}^{+\infty} f(x) \mathrm{d} x, \quad R:=\max \left\{M, 2 \int_{-\infty}^{+\infty}|x| f(x) \mathrm{d} x\right\} .
$$

(In the trivial case that $f=0$, it is understood that the right hand side is also 0.) 
Proof. Again, it is clearly enough to prove it when $M=\int_{-\infty}^{\infty} f=1$, so let us assume this. Call

$$
K:=\int_{-\infty}^{+\infty}|x| f(x) \mathrm{d} x
$$

If we take any $R \geq 2 K$, then

$$
\int_{|x|>R} f(x) \mathrm{d} x \leq \frac{1}{2 K} \int_{\mathbb{R}}|x| f(x) \mathrm{d} x=\frac{K}{2 K}=\frac{1}{2} .
$$

Now, call

$$
m_{R}:=\int_{|x|<R} f(x) \mathrm{d} x .
$$

From the previous bound we know $m_{R} \geq \frac{1}{2}$. Now, using Lemma B.1 we have:

$$
\begin{array}{rl}
\int_{-\infty}^{\infty} f(x) \sin x \mathrm{~d} x=\int_{|x| \leq R} f(x) \sin x \mathrm{~d} x+\int_{|x|>R} & f(x) \sin x \mathrm{~d} x \\
\leq\left(1-\alpha_{1}\right) \int_{|x| \leq R} f(x) \mathrm{d} x & +\int_{|x|>R} f(x) \mathrm{d} x \\
& =\left(1-\alpha_{1}\right) m_{R}+\left(1-m_{R}\right)=1-\alpha_{1} m_{R}
\end{array}
$$

where

$$
\alpha_{1}:=\frac{m_{R}^{4}}{128 \pi n^{2}\|f\|_{L^{2}(-R, R)}^{4}}, \quad n:=1+\frac{R}{2 \pi},
$$

In order to simplify the expression, take $R=: \max \{1,2 K\}$, so that $R \geq 1$ is ensured. Then $n \leq 2 R$ and

$$
\alpha_{1} m_{R} \geq \frac{m_{R}^{5}}{2^{11} R^{2}\|f\|_{L^{2}}^{4}} \geq \frac{1}{2^{16} R^{2}\|f\|_{L^{2}}^{4}}
$$

since we know $m_{R} \geq 1 / 2$.

Now we can complete our main bound, used in the proof of 2.16:

Lemma B.4. Take a nonnegative function $f \in L^{1}(\mathbb{R}) \cap L^{2}(\mathbb{R})$ with $\int_{-\infty}^{\infty}|x| f(x) \mathrm{d} x<+\infty$. Then for all $\xi \in \mathbb{R}$ we have

$$
\left|\int_{-\infty}^{+\infty} f(x) e^{-i x \xi} \mathrm{d} x\right| \leq(1-\alpha) \int_{-\infty}^{+\infty} f(x) \mathrm{d} x
$$

where

$$
\alpha=\alpha(f, \xi):=\frac{\xi^{2} M^{6}}{2^{16} R^{2}\|f\|_{L^{2}}^{4}}, \quad M:=\int_{-\infty}^{+\infty} f(x) \mathrm{d} x, \quad R:=2 \xi \int_{-\infty}^{+\infty}|x| f(x) \mathrm{d} x+2 \pi M .
$$

(In the trivial case that $f=0$, it is understood that the right hand side is also 0.) 
Proof. By the change of variables $y \mapsto x \xi$, it is enough to prove it when $\xi=1$. By scaling $f$ as before, we may also assume that $M=\int_{-\infty}^{\infty} f=1$. We use the following trick to rewrite the modulus as an integral similar to that in Lemma B.3: if we call

$$
a:=\int_{-\infty}^{\infty} f(x) \cos x \mathrm{~d} x, \quad b:=\int_{-\infty}^{\infty} f(x) \sin x \mathrm{~d} x,
$$

then $a^{2}+b^{2}=1$ and there exists some $\theta \in[0,2 \pi)$ such that $a=\sin \theta, b=\cos \theta$.

$$
\begin{aligned}
\left|\int_{-\infty}^{+\infty} f(x) e^{-i x} \mathrm{~d} x\right|^{2} & =\left(\int_{-\infty}^{+\infty} f(x) \cos x \mathrm{~d} x\right)^{2}+\left(\int_{-\infty}^{+\infty} f(x) \sin x \mathrm{~d} x\right)^{2} \\
= & \sin \theta \int_{-\infty}^{+\infty} f(x) \cos x \mathrm{~d} x+\cos \theta \int_{-\infty}^{+\infty} f(x) \sin x \mathrm{~d} x \\
& =\int_{-\infty}^{+\infty} f(x) \sin (x+\theta) \mathrm{d} x=\int_{-\infty}^{+\infty} f(x-\theta) \sin x \mathrm{~d} x .
\end{aligned}
$$

We then apply Lemma B.3 to $\tilde{f}(x):=f(x-\theta)$ to obtain the result. Notice that $\|\tilde{f}\|_{2}=\|f\|_{2}$, $\int_{-\infty}^{\infty} \tilde{f}=\int_{-\infty}^{\infty} f$, and

$$
\int_{-\infty}^{\infty}|x| \tilde{f}(x) \mathrm{d} x=\int_{-\infty}^{\infty}|x+\theta| f(x) \mathrm{d} x \leq \int_{-\infty}^{\infty}|x| f(x) \mathrm{d} x+2 \pi M .
$$

\section{Proof of Lemma 4.6 on the transport semigroup}

Proof of Lemma 4.6. We first show that for each $t \geq 0$ the operator $T_{t}$ is well-defined on the respective spaces, while for $H^{-1}\left(\mathrm{e}^{\mu x}\right)$ we also recall Remark 4.5. In fact, using the change of variables $x \mapsto x \mathrm{e}^{-t}$ we find for $L_{k}^{1}$ that

$$
\begin{aligned}
\left\|T_{t} h\right\|_{L_{k}^{1}}=\int_{0}^{\infty}\left|h\left(x \mathrm{e}^{t}\right)\right|(1+x)^{k} \mathrm{~d} x=\mathrm{e}^{-t} \int_{0}^{\infty}|h(x)| & \left(1+x \mathrm{e}^{-t}\right)^{k} \mathrm{~d} x \\
& \leq \mathrm{e}^{-t} \int_{0}^{\infty}|h(x)|(1+x)^{k} \mathrm{~d} x=\mathrm{e}^{-t}\|h\|_{L_{k}^{1}} .
\end{aligned}
$$

Similarly, we get

$$
\begin{aligned}
\left\|T_{t} h\right\|_{L^{2}\left(\mathrm{e}^{\mu x}\right)}^{2}=\int_{0}^{\infty}\left|h\left(x \mathrm{e}^{t}\right)\right|^{2} \mathrm{e}^{\mu x} \mathrm{~d} x=\mathrm{e}^{-t} \int_{0}^{\infty}|h(x)|^{2} \mathrm{e}^{\mu x \mathrm{e}^{-t}} \mathrm{~d} x & \\
& \leq \mathrm{e}^{-t} \int_{0}^{\infty}|h(x)|^{2} \mathrm{e}^{\mu x} \mathrm{~d} x=\mathrm{e}^{-t}\|h\|_{L^{2}\left(\mathrm{e}^{\mu x}\right)}^{2} .
\end{aligned}
$$

Finally, for $H^{-1}\left(\mathrm{e}^{\mu x}\right)$ we obtain

$$
\begin{gathered}
\left\|T_{t} h_{k}\right\|_{H^{-1}\left(\mathrm{e}^{\mu x}\right)=\int_{0}^{\infty} \mathrm{e}^{\mu x}}\left(\int_{x}^{\infty} h_{k}\left(z \mathrm{e}^{t}\right) \mathrm{d} z\right)^{2} \mathrm{~d} x=\mathrm{e}^{-2 t} \int_{0}^{\infty} \mathrm{e}^{\mu x}\left(\int_{x \mathrm{e}^{t}}^{\infty} h_{k}(z) \mathrm{d} z\right)^{2} \mathrm{~d} x \\
=\mathrm{e}^{-3 t} \int_{0}^{\infty} \mathrm{e}^{\mu x \mathrm{e}^{-t}}\left(\int_{x}^{\infty} h_{k}(z) \mathrm{d} z\right)^{2} \mathrm{~d} x \\
\leq \mathrm{e}^{-3 t} \int_{0}^{\infty} \mathrm{e}^{\mu x}\left(\int_{x}^{\infty} h_{k}(z) \mathrm{d} z\right)^{2} \mathrm{~d} x=\mathrm{e}^{-3 t}\left\|h_{k}\right\|_{H^{-1}\left(\mathrm{e}^{\mu x}\right)}^{2}
\end{gathered}
$$


In particular, this yields the estimates

$$
\begin{aligned}
\left\|T_{t}\right\|_{L_{k}^{1} \rightarrow L_{k}^{1}} & \leq \mathrm{e}^{-t} \\
\left\|T_{t}\right\|_{L^{2}\left(\mathrm{e}^{\mu x}\right) \rightarrow L^{2}\left(\mathrm{e}^{\mu x}\right)} & \leq \mathrm{e}^{-\frac{1}{2} t} \\
\left\|T_{t}\right\|_{H^{-1}\left(\mathrm{e}^{\mu x}\right) \rightarrow H^{-1}\left(\mathrm{e}^{\mu x}\right)} & \leq \mathrm{e}^{-\frac{3}{2} t}
\end{aligned}
$$

for all $t \geq 0$.

It thus remains to verify the strong continuity. By means of [8, Ch.I, 5.3 Proposition] and (C.1) it is sufficient to show that $\lim _{t \rightarrow 0} T_{t} h=h$ for all $h$ in a dense subset $D \subset L^{1}\left((1+x)^{k}\right)$, $D \subset L^{2}\left(\mathrm{e}^{\mu x}\right)$ or $D \subset H^{-1}\left(\mathrm{e}^{\mu x}\right)$ respectively. Thus, taking for example $D=C_{c}^{\infty}((0, \infty))$ we find for $h \in D$ that

$$
\begin{array}{r}
\left\|T_{t} h-h\right\|_{L_{k}^{1}}=\int_{0}^{\infty}\left|h\left(x \mathrm{e}^{t}\right)-h(x)\right|(1+x)^{k} \mathrm{~d} x=\int_{0}^{\infty}\left|\int_{0}^{t} \partial_{\tau} h\left(x \mathrm{e}^{\tau}\right) \mathrm{d} \tau\right|(1+x)^{k} \mathrm{~d} x \\
=\int_{0}^{\infty}\left|\int_{0}^{t} x \mathrm{e}^{\tau} h^{\prime}\left(x \mathrm{e}^{\tau}\right) \mathrm{d} \tau\right|(1+x)^{k} \mathrm{~d} x \leq \int_{0}^{t} \mathrm{e}^{\tau} \int_{0}^{\infty}\left|h^{\prime}\left(x \mathrm{e}^{\tau}\right)\right| x(1+x)^{k} \mathrm{~d} x \mathrm{~d} \tau \\
=\int_{0}^{\infty} \mathrm{e}^{-\tau} \int_{0}^{\infty}\left|h^{\prime}(x)\right| x\left(1+x \mathrm{e}^{-\tau}\right)^{k} \mathrm{~d} x \mathrm{~d} \tau \leq\left\|x h^{\prime}(x)\right\|_{L_{k}^{1}}\left(1-\mathrm{e}^{-t}\right) .
\end{array}
$$

For $t \rightarrow 0$ the right-hand side converges to zero which finally yields the strong continuity for $L^{1}\left((1+x)^{k}\right)$. For $L^{2}\left(\mathrm{e}^{\mu x}\right)$ we argue similarly and get

$$
\begin{aligned}
\left\|T_{t} h-h\right\|_{L^{2}\left(\mathrm{e}^{\mu x}\right)}^{2}=\int_{0}^{\infty}\left|h\left(x \mathrm{e}^{t}\right)-h(x)\right|^{2} \mathrm{e}^{\mu x} \mathrm{~d} x=\int_{0}^{\infty}\left|\int_{0}^{t} \partial_{\tau} h\left(x \mathrm{e}^{\tau}\right) \mathrm{d} \tau\right|^{2} \mathrm{e}^{\mu x} \mathrm{~d} x & \\
& =\int_{0}^{\infty}\left|\int_{0}^{t} x \mathrm{e}^{\tau} h^{\prime}\left(x \mathrm{e}^{\tau}\right) \mathrm{d} \tau\right|^{2} \mathrm{e}^{\mu x} \mathrm{~d} x .
\end{aligned}
$$

Together with Cauchy-Schwartz and the change of variables $x \mapsto x \mathrm{e}^{-\tau}$, we find

$$
\begin{array}{r}
\left\|T_{t} h-h\right\|_{L^{2}\left(\mathrm{e}^{\mu x}\right)}^{2} \leq \int_{0}^{\infty} t \int_{0}^{t} x^{2} \mathrm{e}^{2 \tau}\left|h^{\prime}\left(x \mathrm{e}^{\tau}\right)\right|^{2} \mathrm{~d} \tau \mathrm{e}^{\mu x} \mathrm{~d} x=t \int_{0}^{t} \mathrm{e}^{2 \tau} \int_{0}^{\infty} x^{2}\left|h^{\prime}\left(x \mathrm{e}^{\tau}\right)\right|^{2} \mathrm{e}^{\mu x} \mathrm{~d} x \mathrm{~d} \tau \\
\leq t \int_{0}^{t} \mathrm{e}^{-\tau} \int_{0}^{\infty} x^{2}\left|h^{\prime}(x)\right| \mathrm{e}^{\mu x} \mathrm{~d} x \mathrm{~d} \tau=\left\|x h^{\prime}(x)\right\|_{L^{2}\left(\mathrm{e}^{\mu x}\right)} t\left(1-\mathrm{e}^{-t}\right) .
\end{array}
$$

Again, the right-hand side converges to zero as $t \rightarrow 0$ which proves the strong continuity also for $L^{2}\left(\mathrm{e}^{\mu x}\right)$. Finally, for $H^{-1}\left(\mathrm{e}^{\mu x}\right)$ we get analogously

$$
\begin{aligned}
&\left\|T_{t} h-h\right\|_{H^{-1}\left(\mathrm{e}^{\mu x}\right)}^{2}=\int_{0}^{\infty} \mathrm{e}^{\mu x}\left(\int_{x}^{\infty} h\left(z \mathrm{e}^{t}\right)-h(z) \mathrm{d} z\right)^{2} \mathrm{~d} x \\
&=\int_{0}^{\infty} \mathrm{e}^{\mu x}\left(\int_{x}^{\infty} \int_{0}^{t} \partial_{\tau} h\left(z \mathrm{e}^{\tau}\right) \mathrm{d} \tau \mathrm{d} z\right)^{2} \mathrm{~d} x= \int_{0}^{\infty} \mathrm{e}^{\mu x}\left(\int_{x}^{\infty} \int_{0}^{t} z \mathrm{e}^{\tau} h^{\prime}\left(z \mathrm{e}^{\tau}\right) \mathrm{d} \tau \mathrm{d} z\right)^{2} \mathrm{~d} x \\
&=\int_{0}^{\infty} \mathrm{e}^{\mu x}\left(\int_{0}^{t} \mathrm{e}^{-\tau} \int_{x \mathrm{e}^{\tau}}^{\infty} z h^{\prime}(z) \mathrm{d} z \mathrm{~d} \tau\right)^{2} \mathrm{~d} x .
\end{aligned}
$$


Applying Hölder's inequality we find

$$
\begin{aligned}
\left\|T_{t} h-h\right\|_{H^{-1}\left(\mathrm{e}^{\mu x}\right)}^{2} \leq \int_{0}^{\infty} \mathrm{e}^{\mu x}\left(\int_{0}^{t} \mathrm{e}^{-2 \tau} \mathrm{d} \tau\right)\left(\int_{0}^{t}\left(\int_{x \mathrm{e}^{\tau}}^{\infty} z h^{\prime}(z)\right)^{2} \mathrm{~d} \tau\right) \mathrm{d} x \\
=\frac{1}{2}\left(1-\mathrm{e}^{-2 t}\right) \int_{0}^{\infty} \mathrm{e}^{\mu x} \int_{0}^{t}\left(\int_{x \mathrm{e}^{\tau}}^{\infty} z h^{\prime}(z) \mathrm{d} z\right)^{2} \mathrm{~d} \tau \mathrm{d} x .
\end{aligned}
$$

Since we are interested in the limit $t \rightarrow 0$, we can assume that $t \leq 1$ and thus, for fixed $h \in C_{c}^{\infty}(0, \infty)$ the integral on the right-hand side is bounded. Therefore, for $t \rightarrow 0$ the right-hand side converges to zero which proves the strong continuity also for $H^{-1}\left(\mathrm{e}^{-\mu x}\right)$.

To determine the generator, we take $h \in C_{c}^{\infty}(0, \infty)$ and compute

$$
\lim _{t \rightarrow 0} \frac{1}{t}\left(T_{t} h-h\right)(x)=\lim _{t \rightarrow 0} \frac{1}{t}\left(h\left(x \mathrm{e}^{t}\right)-h(x)\right)=x h^{\prime}(x) .
$$

This shows that $C_{c}^{\infty}(0, \infty) \subset D\left(\mathcal{B}_{1}\right)$ and $\left.\mathcal{B}_{1}\right|_{C_{c}^{\infty}(0, \infty)}=x \partial_{x}$. Thus, to conclude the proof it suffices to prove that $C_{c}^{\infty}$ is a core for $\mathcal{B}_{1}$.

According to [8, Ch. I, 1.7 Proposition], it suffices to verify that $C_{c}^{\infty}(0, \infty)$ is invariant under the action of $\left(T_{t}\right)_{t \geq 0}$ and that $C_{c}^{\infty}(0, \infty)$ is dense in $L^{1}\left((1+x)^{k}\right), L^{2}\left(\mathrm{e}^{\mu x}\right)$ and $H^{-1}\left(\mathrm{e}^{\mu x}\right)$, respectively. Due to the explicit formula $\left(T_{t} h\right)(x)=h\left(x \mathrm{e}^{t}\right)$ the invariance is clear while density is also well-known or clear by construction.

The claim for the spaces $L^{1}\left((1+x)^{k}\right) \cap\left\{\int_{0}^{\infty} x f(x) \mathrm{d} x=0\right\}, L^{2}\left(\mathrm{e}^{\mu x}\right) \cap\left\{\int_{0}^{\infty} x f(x) \mathrm{d} x=0\right\}$ and $H^{-1}\left(\mathrm{e}^{\mu x}\right) \cap\left\{\int_{0}^{\infty} x f(x) \mathrm{d} x=0\right\}$ directly follows by restricting the semigroup once we notice that $\left(T_{t}\right)_{t \geq 0}$ preserves the constraint.

\section{References}

[1] Azmy S. Ackleh. Parameter estimation in a structured algal coagulation-fragmentation model. Nonlinear Anal., 28(5):837-854, 1997.

[2] Azmy S. Ackleh and Ben G. Fitzpatrick. Modeling aggregation and growth processes in an algal population model: analysis and computations. J. Math. Biol., 35(4):480-502, 1997.

[3] Eric J. Allen and Pierre Bastien. On coagulation and the stellar mass spectrum. The Astrophysical Journal, 452:652, Oct 1995.

[4] José A. Cañizo and Bertrand Lods. Exponential convergence to equilibrium for subcritical solutions of the Becker-Döring equations. Journal of Differential Equations, 255(5):905950, September 2013.

[5] José A. Cañizo and Bertrand Lods. Exponential trend to equilibrium for the inelastic Boltzmann equation driven by a particle bath, July 2015.

[6] José A Cañizo and Stéphane Mischler. Regularity, local behavior and partial uniqueness for Smoluchowski's coagulation equation. Revista Matemática Iberoamericana, 27(3):803839, 2011.

[7] José A. Cañizo, Stéphane Mischler, and Clément Mouhot. Rate of convergence to selfsimilarity for Smoluchowski's coagulation equation with constant coefficients. SIAM Journal on Mathematical Analysis, 41(6):2283-2314, 2010. 
[8] Klaus-Jochen Engel and Rainer Nagel. One-parameter semigroups for linear evolution equations, volume 194 of Graduate Texts in Mathematics. Springer-Verlag, New York, 2000. With contributions by S. Brendle, M. Campiti, T. Hahn, G. Metafune, G. Nickel, D. Pallara, C. Perazzoli, A. Rhandi, S. Romanelli and R. Schnaubelt.

[9] M. Escobedo and S. Mischler. Dust and self-similarity for the Smoluchowski coagulation equation. Ann. Inst. H. Poincaré Anal. Non Linéaire, 23(3):331-362, 2006.

[10] M. Escobedo, S. Mischler, and B. Perthame. Gelation in coagulation and fragmentation models. Comm. Math. Phys., 231(1):157-188, 2002.

[11] M. Escobedo, S. Mischler, and M. Rodriguez Ricard. On self-similarity and stationary problem for fragmentation and coagulation models. Ann. Inst. H. Poincaré Anal. Non Linéaire, 22(1):99-125, 2005.

[12] Nicolas Fournier and Philippe Laurençot. Existence of self-similar solutions to Smoluchowski's coagulation equation. Comm. Math. Phys., 256(3):589-609, 2005.

[13] Nicolas Fournier and Philippe Laurençot. Local properties of self-similar solutions to Smoluchowski's coagulation equation with sum kernels. Proc. Roy. Soc. Edinburgh Sect. A, 136(3):485-508, 2006.

[14] Sheldon K. Friedlander. Smoke, Dust, and Haze: Fundamentals of Aerosol Dynamics. Topics in Chemical Engineering. Oxford University Press, 2000.

[15] Maria P. Gualdani, Stéphane Mischler, and Clément Mouhot. Factorization for nonsymmetric operators and exponential H-theorem. Mémoires de la Société Mathématique de France, 2018. To appear.

[16] Philippe Laurençot. Uniqueness of Mass-Conserving Self-similar Solutions to Smoluchowski's Coagulation Equation with Inverse Power Law Kernels. J. Stat. Phys., 171(3):484-492, 2018.

[17] Philippe Laurençot, Barbara Niethammer, and Juan J. L. Velázquez. Oscillatory dynamics in Smoluchowski's coagulation equation with diagonal kernel. Kinet. Relat. Models, 11(4):933-952, 2018.

[18] Govind Menon and Robert L. Pego. Approach to self-similarity in Smoluchowski's coagulation equations. Communications on Pure and Applied Mathematics, 57(9):1197-1232, 2004.

[19] Govind Menon and Robert L. Pego. Dynamical scaling in Smoluchowski's coagulation equations: uniform convergence. SIAM J. Math. Anal., 36(5):1629-1651, 2005.

[20] S. Mischler and C. Mouhot. Cooling process for inelastic Boltzmann equations for hard spheres. II. Self-similar solutions and tail behavior. J. Stat. Phys., 124(2-4):703-746, 2006.

[21] S. Mischler and C. Mouhot. Stability, convergence to self-similarity and elastic limit for the Boltzmann equation for inelastic hard spheres. Communications in Mathematical Physics, 288(2):431-502, June 2009. 
[22] S. Mischler and C. Mouhot. Exponential stability of slowly decaying solutions to the kinetic-Fokker-Planck equation. Archive for Rational Mechanics and Analysis, 221(2):677-723, Aug 2016.

[23] S. Mischler, C. Mouhot, and M. Rodríguez Ricard. Cooling Process for Inelastic Boltzmann Equations for Hard Spheres, Part I: The Cauchy Problem. Journal of Statistical Physics, V124(2):655-702, 2006.

[24] Clément Mouhot. Rate of convergence to equilibrium for the spatially homogeneous Boltzmann equation with hard potentials. Communications in Mathematical Physics, 261(3):629-672, February 2006.

[25] B. Niethammer, S. Throm, and J. J. L. Velázquez. A revised proof of uniqueness of self-similar profiles to Smoluchowski's coagulation equation for kernels close to constant. Preprint arXiv:1510.03361v3, October 2015.

[26] B. Niethammer, S. Throm, and J. J. L. Velázquez. Self-similar solutions with fat tails for Smoluchowski's coagulation equation with singular kernels. Ann. Inst. H. Poincaré Anal. Non Linéaire, 33(5):1223-1257, 2016.

[27] B. Niethammer, S. Throm, and J. J. L. Velázquez. A uniqueness result for self-similar profiles to Smoluchowski's coagulation equation revisited. J Stat Phys, 164(2):399-409, Jun 2016.

[28] B. Niethammer and J. J. L. Velázquez. Self-similar solutions with fat tails for Smoluchowski's coagulation equation with locally bounded kernels. Comm. Math. Phys., 318(2):505-532, 2013.

[29] B. Niethammer and J. J. L. Velázquez. Exponential tail behavior of self-similar solutions to Smoluchowski's coagulation equation. Comm. Partial Differential Equations, 39(12):2314$2350,2014$.

[30] B. Niethammer and J. J. L. Velázquez. Uniqueness of self-similar solutions to Smoluchowski's coagulation equations for kernels that are close to constant. Journal of Statistical Physics, 157(1):158-181, 2014.

[31] H.R. Pruppacher and J.D. Klett. Microphysics of Clouds and Precipitation. Springer Netherlands, 2010.

[32] J. Silk and T. Takahashi. A statistical model for the initial stellar mass function. The Astrophysical Journal, 229:242, Apr 1979.

[33] R. Srinivasan. Rates of convergence for Smoluchowski's coagulation equations. SIAM Journal on Mathematical Analysis, 43(4):1835-1854, 2011.

[34] Sebastian Throm. Uniqueness of fat-tailed self-similar profiles to Smoluchowski's coagulation equation for a perturbation of the constant kernel. Preprint arXiv:1704.01949 (to appear in Memoirs of the AMS), 2017.

[35] Sebastian Throm. Stability and uniqueness of self-similar profiles in $L^{1}$ spaces for perturbations of the constant kernel in Smoluchowski's coagulation equation. Preprint arXiv:1902.10000, 2019. 